\title{
Using catch-per-unit-effort \\ data to solve spatial problems in Orange Roughy abundance estimates
}

\author{
by \\ Max Schofield
}

A thesis

submitted to the Victoria University of Wellington in fulfilment of the requirements for the degree of

Master of Science

in Biological Sciences.

Victoria University of Wellington

2015 



\begin{abstract}
This thesis describes a thorough analysis of the Andes Complex orange roughy fishery, which started in 1991 and continues to date. The Andes Complex orange roughy fishery displays a rapid initial decline in catch rate, followed by a prolonged period of relatively stable catch rate. This trend is the classic feature of a hyperdepletion catch rate. The trends in the observed Andes Complex orange roughy catch rates were explored through the development of eight modified Schaefer Surplus Production Models (SPM). Each model applied a hypothesis about a mechanism catalysing the observed trend of the fishery. The SPM was modified by either adding new information to the model, or an additional parameter. The fits of the modified models were optimised to elucidate values of unknown parameters in the SPM, and these were used to create estimated abundance indicies for each model. Then I compared each index to the observed abundance index (catch rate), derived following an Exploratory Analysis. The best candidate models, which had the smallest likelihoods, $B I C$ values, and best visual fits, were those assuming population growth rate changed midway through the fishery, or that the population size decreased following habitat damage (from trawling).
\end{abstract}




\section{Acknowledgments}

I would like to thank MPI (Ministry for Primary Industries) and NIWA (National Institute of Water and Atmospheric Research) for their support of my post-graduate study through the provision of a post-graduate scholarship in Quantitative Fisheries Science. I am eternally greatful for the guidance and advice provided by my two supervisors Matt Dunn and Nokuthaba Sibanda. Thanks to Craig for coming along for the ride and help when I hit a wall. Thanks to Claudia for your support and encouragement. Finally, thanks to Mum and Dad for your help all along the way. 


\section{Contents}

Acknowledgments

1 Introduction 3

1.1 Catch Per Unit Effort . . . . . . . . . . . . . . . 5

1.2 Spatial Structure of Fish Populations . . . . . . . . . . . . 10

1.3 Orange Roughy . . . . . . . . . . . . . . . . . . . 15

1.4 Objective and Approach . . . . . . . . . . . . . . 17

1.5 Hypotheses for Stock Structure . . . . . . . . . . . . . . 18

2 Model Structure and Estimation Methods 21

2.1 Surplus Production Model . . . . . . . . . . . . . . . . . 21

2.1.1 Schaefer Population Growth Model . . . . . . . . . 23

2.1.2 Model Equations . . . . . . . . . . . . . . 27

2.1.3 Model Errors . . . . . . . . . . . . . . . . 31

2.2 Maximum Likelihood Estimation . . . . . . . . . . . . . 34

2.3 Parameter Estimation . . . . . . . . . . . . . . . . 36

2.4 Generalised Linear Models . . . . . . . . . . . . . . . . . . . 39

3 Exploratory Analysis of the Andes Fishery 43 
3.1 Andes Complex . . . . . . . . . . . . . . . . . 44

3.2 Analysis .......................... 45

3.3 History of the Andes Fishery . . . . . . . . . . . . 46

3.4 Temporal Structure of the Andes Fishery . . . . . . . . . 48

3.5 Vessels .......................... 50

3.6 Subareas ......................... 54

3.7 Generalised Linear Modeling . . . . . . . . . . . . . 59

3.8 Andes Model . . . . . . . . . . . . . . . . . . . . 61

3.8.1 Binomial Model . . . . . . . . . . . . . . . . 62

3.8.2 Normal Model . . . . . . . . . . . . . . 65

3.8.3 Combined Model . . . . . . . . . . . . . . . . . . 68

3.8.4 Final Model . . . . . . . . . . . . . . . . . . . 72

4 Model Fitting and Comparison $\quad 77$

4.1 Model Assessment . . . . . . . . . . . . . . . 77

4.1.1 Information Criteria $\ldots \ldots \ldots 77$

4.1.2 Confidence Intervals and Likelihood Profiles . . . . . 79

4.1 .3 Visual Assessment . . . . . . . . . . . . . 81

4.2 Hypothesised Models . . . . . . . . . . . . . . . . . . . 82

4.2.1 Closed Population Model . . . . . . . . . . . . . 82

4.2.2 The Changing Growth Model . . . . . . . . . . . 87

4.2.3 The Altered Environment Model . . . . . . . . . . . . 89

4.2.4 The Disturbance Model . . . . . . . . . . . . . . 94

4.2.5 The Habitat Degradation Model . . . . . . . . . . 96

4.3 Model Evaluation . . . . . . . . . . . . . . . . . . . . 101

5 Discussion 105 
6 Appendix 115

6.1 Appendix A: R code . . . . . . . . . . . . . . 115

6.1.1 Example: Unweighted Habitat Degradation Model . 115

6.2 Appendix B: Likelihood profiles . . . . . . . . . . . . . . . 121

6.2.1 Changing Growth Model . . . . . . . . . . . . . . . 121

6.2.2 Unweighted Habitat Degradation Model . . . . . . . 122 


\section{List of Figures}

1.1 Three possible relationships between CPUE and abundance as described by Hilborn and Walters (1992) . . . . . . . . . 11

2.1 Logistic growth curve of the Schaefer model (left); and population growth $\left(r B_{t}\left(1-\frac{B_{t}}{K}\right)\right)$ as biomass increases (right). . . 24

2.2 The grid for the Andes Complex used to create the index of habitat damaged $(H D)$; each grid cell is $0.05 \times 0.05$ degree latitude longitude bins. Tows were allocated to grid cells based on the tow starting co-ordinates. The dots represent the peak of the each of the Andes Complex seamounts. . . . 30

2.3 The probability density curve for the catch data. . . . . . . . 40

3.1 The Eastern Chatham Rise orange roughy fishery; composed of eight distinct areas, A: the Graveyard Complex B: The Spawning Box, C: The Northeast Hills, D: Kenwood, E: The Andes Complex, F: The Middleground, G: Chiefs \& Neighbours and H: Hedgerville as defined in Anderson \& Dunn 2012. Seamount features are plotted with blue dots marking their peaks, the proposed population boundary between the two Chatham orange roughy populations from Dunn \& Devine (2010) is shown by the dashed line, and the $1000 \mathrm{~m}$ depth contour has been added. . . . . . . . . . . . . . . . . . . 44

3.2 The Andes Complex; coloured bathymetry indicates depth (from $600 \mathrm{~m}$ to $2000 \mathrm{~m}$ ); hill peaks are indicated by blue dots, hill names are adjacent to peaks $(10 \mathrm{~nm}=10$ nautical miles).

3.3 History of the Andes Seamount Complex orange roughy fishery from 1989 to 2013. A, Catch; B, Effort; C, Raw CPUE; $\mathrm{D}$, Cumulative catch. . . . . . . . . . . . . . . . 48 
3.4 Left: The proportion of zero tows (not capturing roughy) from the fishery and right The number of vessels fishing in the Andes Complex between 1989 and 2013 . . . . . . . . . . 51

3.5 The fishing patterns of the six vessels targeting orange roughy at the Andes Complex in 1992. Each vessel has an individual plot with a unique colour for its towlines. Every tow conducted is plotted with black dots indicating the end position of each tow. . . . . . . . . . . . . . 53

3.6 The geographical Andes Complex is identified and segregated into four subareas (top left). Each tow recorded in the wider Andes area from 1991 to 2013 has been plotted with start and end positions, with blue dots to show tow direction (top right). Each of the four subareas: northwest (middle left), east (middle right), southwest (bottom left) and south (bottom right), are plotted with all of their tows. A tow was allocated to an area based on its starting position. 56

3.7 Left: Raw CPUE (tonnes/tow) of the four areas of the Andes and Right: the cumulative catch of these regions. The South Andes is plotted black, the East Andes green, the Northwest Andes blue and the Southwest Andes red. . . . . 58

3.8 Four alternate area splits offered to the model for selection: no split (top left), north vs south (top right), east vs west (bottom left), and big seamounts vs small seamounts (bottom right). . . . . . . . . . . . . . . . . 59

3.9 Observed and expected proportions of non-zero fits of the final Andes Binomial model. . . . . . . . . . . . . . . . 63

3.10 Binomial GLM predictions for the overall binomial index from 1991 to 2013 (top left), the vessel effect (top right) and the subarea effect (bottom left). Subareas are denoted: EA east Andes, NWA northwest Andes, SA south Andes and SWA southwest Andes. Each effect has been plotted with the other parameters set to their median value and with their corresponding $95 \%$ confidence intervals. . . . . . . . . . 64

3.11 Normal quantile-quantile plot for the final Andes normal model fit. . . . . . . . . . . . . . . 65 
3.12 Normal Generalised Linear Model predictions; south Andes (top left), southwest Andes (top right), northwest Andes (middle left), east Andes (middle right) from 1991-2013; vessel effects (bottom left) and subarea effects (bottom right). Subareas are denoted: EA east Andes, NWA northwest Andes, SA south Andes and SWA southwest Andes. Each effect has been plotted with the other parameters set to their median value and with its corresponding $95 \%$ confidence intervals. . . . . . . . . . . . . . .

3.13 Final abundance indicies for the combined model; year from 1991 to 2013 (top left), vessel (top right), and subarea (bottom). Each effect is plotted with the other parameters set at their median values and with its corresponding bootstrapped coefficients of variation. . . . . . . . . . . .

3.14 Comparison between raw unstandardised CPUE (blue) and the untransformed normal GLM predictions (x) with their corresponding 95\% confidence intervals (dotted lines) for the Andes four subareas; south (top left), east (top right), northwest (bottom left), and south west (bottom right), from 1991 to 2013. The third axis displays the catch of orange roughy (tons), which are plotted at squares as the bottom of the graph. . . . . . . . . . . . . .

3.15 Comparison of the differences in abundance indices between the four Andes subareas from 1991 to 2013: South Andes (black), East Andes (red), Northwest Andes (green) and Southwest Andes (blue). The subareas have been standardised, then scaled to the geometric mean of the East Andes; the CV for the East Andes has been added to the plot. . . . . . .

3.16 Final model output for the Andes Complex, binomial model, first row, normal model, second row and combined model bottom row. The model predictions are plotted with their corresponding CV's; these are bootstrapped for the combined model. Predictions are based on using the Vessel ID 10227 for Year and the Year 2002 for Vessel ID . . . . . . . . .

4.1 Minimum log-likelihood profiles of $\mathrm{r}$ from $0-0.5$ (bottom); and $\mathrm{K}$ from 14000-26000 tons. The left hand panels are standard profiles whereas the right hand panels have a penalty added to the likelihood function. . . . . . . . . . . 
4.2 Estimated Andes orange roughy population index (blue) with corresponding 95\% confidence intervals for the Closed Population Model and the observed population index (black) from 1991 - 2013 (left). The residual plot shows the difference between the estimated and observed index; the central dashed line indicates no difference (zero) and the periphery dashed lines indicate $+/-2$ standard deviations of the residuals. The top panels show the unmodified abundance index, whereas the bottom panels have a penalty added to the likelihood function. . . . . . . . . . . . . . . . . . .

4.3 Retrospective fit of the Closed Population Model of the Andes Complex orange roughy fishery. The observed abundance index (black) is compared to the closed population model with four different lengths: the full dataset (purple); 5 years removed (green); 10 years removed (blue); and 15

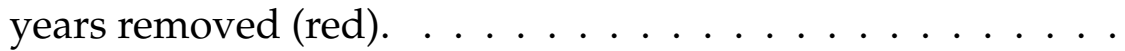

4.4 Estimated Andes orange roughy population index (blue) with corresponding 95\% confidence intervals for the Changing Growth Model and the observed population index (black) from 1991-2013. The vertical dashed line indicates the transition point between growth rates. The residual plot shows the difference between the estimated and observed index, the central dashed line indicates no difference (zero) and the periphery dashed lines indicate $+/-2$ standard deviations of the residuals. . . . . . . . . . . . . . . .

4.5 The mean annual sea surface temperature values for the Andes Complex and the corresponding values of growth rate $r$ and $95 \%$ confidence intervals generated by using the parameter estimates of $m$ and $c$ from the Altered Environment Model $(r) \ldots \ldots \ldots \ldots \ldots$

4.6 Estimated Andes orange roughy population index (blue) with corresponding 95\% confidence intervals for the Altered Environment Model $(r)$ and the observed population index (black) from $1991-2013$. The residual plot shows the difference between the estimated and observed index; the central dashed line indicates no difference (zero) and the periphery dashed lines indicate $+/-3$ standard deviations of the residuals. . . . . . . . . . . . . . . . 
4.7 The mean annual sea surface temperature values for the Andes Complex and the corresponding values of carrying capacity $K$ and its $95 \%$ confidence interval, generated by using the parameter estimates of $m$ and $c$ from the Altered Environment Model $(K) \ldots \ldots \ldots \ldots$

4.8 Estimated Andes orange roughy population index (blue) with corresponding $95 \%$ confidence intervals for the Altered Environment Model $(K)$ and the observed population index (black) from 1991 - 2013. The residual plot shows the difference between the estimated and observed index, the central dashed line indicates no difference (zero) and the periphery dashed lines indicate $+/-3$ standard deviations of the residuals. . . . . . . . . . . . . . . .

4.9 Estimated Andes orange roughy population index (blue) with corresponding 95\% confidence intervals for the Disturbance Model and the observed population index (black) from $1991-2013$. The residual plot shows the difference between the estimated and observed index; the central dashed line indicates no difference (zero) and the periphery dashed lines indicate $+/-3$ standard deviations of the residuals. . .

4.10 The annual number of tows at the Andes Complex (left) and the disturbance index that was applied to the carrying capacity (right), from 1991 to 2013. . . . . . . . . . . . . . .

4.11 Vectors of unweighted and weighted habitat damage for the Andes Complex. Unweighted habitat damage assumes all the grid has equal value, whereas the weighted habitat gives grid cells with seamounts an order of magnitude more value. . . . . . . . . . . . . . .

4.12 Estimated Andes orange roughy population index (blue) with corresponding 95\% confidence intervals for the Unweighted Habitat Degradation Model and the observed population index (black) from 1991 - 2013. The residual plot shows the difference between the estimated and observed index; the central dashed line indicates no difference (zero) and the periphery dashed lines indicate $+/-2$ standard deviations of the residuals. . . . . . . . . . . . . . 
4.13 Estimated Andes orange roughy population index (blue) with corresponding $95 \%$ confidence intervals for the Transformed Habitat Degradation Model and the observed population index (black) from $1991-2013$. The residual plot shows the difference between the estimated and observed index; the central dashed line indicates no difference (zero) and the periphery dashed lines indicate $+/-2$ standard deviations of the residuals. . . . . . . . . . . . . . . . 100

4.14 Estimated Andes orange roughy population index (blue) with corresponding 95\% confidence intervals for the Weighted Habitat Degradation Model and the observed population index (black) from $1991-2013$. The residual plot shows the difference between the estimated and observed index; the central dashed line indicates no difference (zero) and the periphery dashed lines indicate $+/-3$ standard deviations of the residuals. . . . . . . . . . . . . . . . . . 101

4.15 A comparison of the observed (black) and expected (blue) abundance indicies of the two best candidate models for the Andes Complex orange roughy fishery, the Changing Growth Model (left) and the Unweighted Habitat Degradation Model (right). Both models are plotted with their corresponding 95\% confidence intervals (dashed lines) and the Changing Growth Model has a vertical line corresponding to the transition point between the two growth rates. . . . 103

6.1 Minimum negative log-likelihood profiles for biological parameters $r 2$ and $K$ from the Changing Growth Model ( $r 1$ was $\sim 0$ so it was not profiled). . . . . . . . . . . . 121

6.2 Minimum negative log-likelihood profiles for parameters K and $\mathrm{r}$ for the Unweighted Habitat Degradation Model. . . . 122 


\section{List of Tables}

1 Glossary of fisheries terms from United Nations Food and Agriculture Organisation $(\mathrm{FAO}) \ldots \ldots \ldots$

2 Fisheries Abbreviations . . . . . . . . . . . . . 2

1.1 Hyperstable and Hyperdeplete Fisheries. . . . . . . . . . . . 13

3.1 The number of tows in each month at the Andes Complex from $1989-2013 \ldots \ldots$. . . . . . . . . . . . 50

3.2 The potential predictors offered to the Generalised Linear Models ....................... 61

3.3 A summary of the data that goes into my GLM; the data is separated by subarea. For each subarea (EA east Andes, NWA northwest Andes, SA south Andes, SWA southwest Andes) the annual number of tows $(n)$, number of tows that capture ORH $(n($ orh $))$ and median weight of catch $(\mathrm{kg})$ are displayed. Tows from outside the Andes (Other) are shown to illustrate the lack of data available to form an abundance index. . . . . . . . . . . . . . 62

3.4 Model selection for the Binomial GLM . . . . . . . . . . . . . 62

3.5 Model selection for Normal GLM . . . . . . . . . . . . . . . 65

3.6 Reanalysis of selection for Normal model . . . . . . . . . . . 72

3.7 The final Binomial Model . . . . . . . . . . . . . . 72

3.8 The final Normal Model . . . . . . . . . . . . . . . . . 73

3.9 Final GLM table; binomial, normal and combined models displayed with their Coefficients of Variation $(\mathrm{CVs}) \quad \ldots .75$ 
4.1 Parameter estimates and their corresponding 95\% confidence intervals for Closed Population Model, estimates with the subscript $p$ have a penalty added to their likelihood. ( ${ }^{*}$ parameter has hit a bound) . . . . . . . . . . . . 83

4.2 The likelihood values for a range of transition years between growth rates .................. . . 87

4.3 Parameter estimates and their corresponding 95\% confidence intervals for the Changing Growth Model . . . . . . . . . 88

4.4 Parameter estimates and their corresponding 95\% confidence intervals for the Altered Environment Model $(r) \ldots . . . .90$

4.5 Parameter estimates and their corresponding $95 \%$ confidence intervals for the Altered Environment Model $(K)$. . . . . . 92

4.6 Parameter estimates and their corresponding 95\% confidence intervals for the Disturbance Model, $*$ indicates the estimate has hit a bound. . . . . . . . . . . . . . . . . . 94

4.7 Parameter estimates and their corresponding 95\% confidence intervals for the Unweighted Habitat Model . . . . . . . . 97

4.8 Parameter estimates and their corresponding 95\% confidence intervals for the Transformed Habitat Degradation Model . . 99

4.9 Parameter estimates and their corresponding 95\% confidence intervals for the Weighted Habitat Degradation Model . . . 100

4.10 Model Evaluation Table: each model with its corresponding Likelihood, Number of Parameters, AIC, AIC_c, BIC and MSY102 
Table 1: Glossary of fisheries terms from United Nations Food and Agriculture Organisation $(\mathrm{FAO})$

\begin{tabular}{|c|c|}
\hline Term & Definition \\
\hline Aggregation & $\begin{array}{l}\text { The process of grouping (of fish) for various behavioral reasons related to } \\
\text { migration, spawning or feeding }\end{array}$ \\
\hline Benthic & $\begin{array}{l}\text { Refers to animals and fish that live on or in the bottom water layer } \\
\text { or in the sediment }\end{array}$ \\
\hline Benthopelagic & $\begin{array}{l}\text { Living and feeding near the bottom as well as in midwaters or } \\
\text { near the surface. Feeding on benthic as well as free swimming organisms. }\end{array}$ \\
\hline Biomass (B) & $\begin{array}{l}\text { The total weight of a group or stock of living organisms or of some } \\
\text { defined fraction of it (e.g. spawners), in an area, at a particular time }\end{array}$ \\
\hline By-catch & $\begin{array}{l}\text { Part of a catch of a fishing unit taken incidentally in addition to the target } \\
\text { species towards which fishing effort is directed }\end{array}$ \\
\hline Carrying Capacity (K) & $\begin{array}{l}\text { The maximum population of a species that a specific ecosystem can } \\
\text { support indefinitely without deterioration of the character and quality } \\
\text { of the resource }\end{array}$ \\
\hline Catch $(\mathrm{C})$ & The total weight of fish caught by fishing operations, estimated on board \\
\hline Catch-per-unit-effort & CPUE: The amount of catch that is taken per unit of fishing effort \\
\hline Catchability (q) & The extent to which a stock is susceptible to fishing \\
\hline Demersal & Living in close relation with the bottom and depending on it \\
\hline Discard & $\begin{array}{l}\text { To release or return fish to the sea, dead or alive, whether or not such fish } \\
\text { are brought fully on board a fishing vessel }\end{array}$ \\
\hline Ecosystem & $\begin{array}{l}\text { A spatio-temporal system of the biosphere, including its living } \\
\text { components and the non-living components of their environment }\end{array}$ \\
\hline Feature & $\begin{array}{l}\text { A location of different topography: hills, seamounts, knolls, canyons and } \\
\text { ridges }\end{array}$ \\
\hline Intrinsic growth rate $(r)$ & $\begin{array}{l}\text { A value that quantifies how much a population can grow between } \\
\text { successive time periods }\end{array}$ \\
\hline Landings & $\begin{array}{l}\text { The weight of what is landed at a landing site. May be different from } \\
\text { the catch }\end{array}$ \\
\hline Metapopulation & $\begin{array}{l}\text { A set of populations that can effectively be, separate, weakly } \\
\text { coupled, or globally interacting, through strongly coupled patches }\end{array}$ \\
\hline Fishing Effort (E) & $\begin{array}{l}\text { The amount of fishing gear of a specific type used on the fishing } \\
\text { grounds over a given unit of time }\end{array}$ \\
\hline Pelagic & $\begin{array}{l}\text { Organisms that spend most of their life in the water column, experience } \\
\text { little contact with, or dependency on, the bottom. }\end{array}$ \\
\hline Population & $\begin{array}{l}\text { A group of interbreeding organisms that represent the level of } \\
\text { organization at which speciation begins }\end{array}$ \\
\hline Population Size $(\mathrm{N})$ & The number of individuals in a population \\
\hline Seamount & $\begin{array}{l}\text { Any geographically isolated topographical feature on the seafloor } \\
\text { taller than } 100 \mathrm{~m}\end{array}$ \\
\hline Stock & $\begin{array}{l}\text { The part of a fish population which is under consideration from the } \\
\text { point of view of actual or potential utilization }\end{array}$ \\
\hline Stock Assessment & $\begin{array}{l}\text { Collecting and analysing biological and statistical information to } \\
\text { determine the changes in the abundance of fishery stocks in response } \\
\text { to fishing }\end{array}$ \\
\hline Stakeholder & $\begin{array}{l}\text { An individual or group of individuals with an interest or claim } \\
\text { who could potentially be impacted by, or have an impact on, a } \\
\text { given project and its objectives }\end{array}$ \\
\hline Sub-populations & $\begin{array}{l}\text { Geographically or otherwise distinct groups in the population } \\
\text { between which there is little demographic exchange }\end{array}$ \\
\hline
\end{tabular}


Table 2: Fisheries Abbreviations

\begin{tabular}{ll} 
Term & Definition \\
\hline AIC & Akaikes Information Criteria \\
BOE & Black Oreo Dory \\
CPUE & Catch Per Unit Effort \\
CR & Chatham Rise \\
ECR & East Chatham Rise \\
EEZ & Exclusive Economic Zone \\
GLM & General Linear Model \\
MPI & Ministry for Primary Industries \\
MSY & Maximum Sustainable Yield \\
NIWA & National Institute for Water and Atmospheric Research \\
ORH & Orange Roughy \\
QMS & Quota Management System \\
SSO & Smooth Oreo dory
\end{tabular}




\section{Chapter 1}

\section{Introduction}

Deep-sea fisheries have been occurring around the world since the 1950's (Roberts, 2002). Deep-sea fisheries refer to any fisheries $500 \mathrm{~m}$ or deeper, below the upper continental shelf (Moore, 1999; Koslow, 2000). The movement of fishers to target deeper fisheries and species was catalysed by the depletion of inshore fish stocks, the development of technology to enable fishing this depth, and the dense commercially valuable fish aggregations available (Koslow, 2000).

The species targeted by deep-sea fisheries are generally slow growing, have very low natural mortality, extreme longevity, and late maturation (Clark, 2009; Norse et al., 2012). These life history characteristics provide a challenge for successful conservation and management (Koslow, 2000). Deep-sea fisheries are notorious for their rapid development and equally rapid resource depletion (Koslow, 2000; Francis and Clark, 2005; Rowden et al., 2010). Early failings of deep-sea fisheries can be attributed to overly optimistic expectations of sustainable yields, poor regulation, the tragedy of the commons and a slow response to scientific advice (Sissenwine and 
Mace, 2007). Deep-sea fisheries are difficult to manage, as a consequence of their nature and the focal organisms are difficult to access (Francis and Clark, 2005).

Stock assessments provide a scientific evaluation of the status of a fish stock and its potential yield (Sissenwine and Mace, 2007). Stock assessments generally collate information about stock structure, population demographics, fisheries-dependent information and fisheries independent information into a model that is used to set fisheries benchmarks (Sissenwine and Mace, 2007). Fisheries benchmarks are stock abundance, and long term sustainable yields. The idea of fisheries stock assessments is to gauge the current population status of a stock, relative to management benchmarks, and adjust catches accordingly to ensure sustainable management of the stock. Deep-sea species management regimes typically have to operate on a low level of knowledge and management action should be cautious as a consequence (Clark and Rowden, 2009). This lack of scientific information leads to many deep-sea fisheries stock assessments being based primarily or solely on commercial catch-per-uniteffort data. Catch-per-unit-effort is used to construct fisheries abundance indices on the assumption that CPUE is proportional to population abundance (Dunn et al., 2000). This assumption of a proportional relationship is highly criticised, especially its poor application in deep-sea and spatially structured fisheries (Maunder et al., 2006; Walters, 2003; Dunn et al., 2000; Campbell, 2015; Clark et al., 2010). Alternate non-linear relationships are thought to occur, but the drivers of these relationships are poorly understood (Hicks, 2013). This thesis uses historical catch-per-unit-effort (CPUE) data of a New Zealand orange roughy fishery, obtained from the 
New Zealand Ministry for Primary Industries (MPI), to investigate and model hypotheses about non-linear relationships between CPUE and population abundance.

\subsection{Catch Per Unit Effort}

Catch Per Unit Effort (CPUE) is the catch extracted from one unit of fishing effort. Catch refers to the estimated number or weight of fish. Units of effort can be variable, for example, per hook or per day. CPUE provides a standardised measure of the relative catch rates, with changes in CPUE assumed to correspond to proportional changes in the abundance of the population (Dunn et al., 2000). For close to a century CPUE has been used by fisheries scientists as an index of population abundance (Seber, 1982). This is a consequence of CPUE being the most readily available piece of information for fisheries stock assessments because it is gathered with all fishing activity. The catch $(C)$ is the product of: the catchability coefficient $(q)$, the fraction of abundance that is captured by one unit of effort; the effort expended by the fleet $(E)$; and the abundance of the fish population $(B)$.

$$
C=q E B
$$

The equation for catch, $C$ (eq. 1.1), can be rearranged for CPUE $C / E$, which is the product of the abundance of the population and the catchability coefficient (eq. 1.2) ( $q B$ ), and implies a proportional (linear) relation- 
ship between $C / E$ and $B$.

$$
\frac{C}{E}=q B
$$

It is important to recognise that CPUE is an index of the population vulnerable to fishing gear (Maunder et al., 2006). The use of CPUE as an index of abundance is often criticised, with many scientists preferentially using fisheries independent indices of abundance, especially when aggregations are targeted (Erisman et al., 2011). The information about abundance encompassed in CPUE is confounded by many other factors, for example, the variable efficiency of different vessels within the fleet (Hilborn, 1985). Confounding factors need to be removed to allow the formation of an unbiased index of abundance (Campbell, 2004). However, for many fisheries, CPUE is the only available index of abundance (Clark, 2010). CPUE is commonly standardised by utilizing a generalized linear model (GLM) or generalized additive model (GAM), to estimate the effect of a single index, usually year, on CPUE (Gavaris, 1980). Standardisation removes exogenous variation and allows for the retention of the proposed proportional relationship assumed between CPUE and relative abundance (Hilborn and Walters, 1992; Walters, 2003; Bordalo-Machado, 2006).

Another assumption of CPUE as an index of abundance is that fishing technology remains consistent (Hilborn, 1985). In long standing fisheries, technological changes are a reality as fishing vessels work to maximize their efficiency. In deep-sea fisheries, as technology has advanced, the fishing fleet has gained the ability to move off flat ground and target "hills" (Clark, 1999). The introduction of more advanced Global Positioning Sys- 
tem (GPS) and echo-sounder technology enabled the industry to locate seamounts and their fish aggregations (Doonan et al., 2009; Clark and Dunn, 2012). Deep-sea fisheries are progressively focusing on targeting seamounts. In 1984, only $30 \%$ of deep-sea fishing was conducted at seamounts but by the 1990's, this increased to 60-70\% (Clark and O'Driscoll, 2003). Coupled with the change in technology, is the learning of skippers, who become more efficient at capturing fish at a location with more experience (Hilborn, 1985). The change in CPUE resulting from this knowledge and technology creep needs consideration when forming a CPUE abundance index. It has been suggested that incorporating more knowledge about gear into model parameters could achieve this (Marchal et al., 2007), or alternatively, splitting the time series could allow for a major technological step change like the introduction of GPS (Doonan et al., 2009).

If fishing effort is spread over a large area it becomes hard to distinguish the populations being fished, especially when population structure is unknown (Cadrin et al., 2013). In contrast, if an area is small, for example, a cluster of seamounts, it may not contain a discrete closed population. A CPUE index formed from a sub-population may not reflect the wider population's demographics (Clark and Dunn, 2012), where the wider population and the sub-populations interact their respective index trends may be dissimilar (Cadrin et al., 2013). This problem is amplified when subpopulation CPUE is not proportional to sub-population abundance, and in this situation the index will poorly represent the wider population.

Fish distribution can be changed by factors other than removal in the form of catch. Bottom trawls endeavour to extract a target species, but they do not discriminate, and also impact the wider marine ecosystem 
(Clark, 2010). The bottom of the trawl is fitted with steel bobbins designed to roll over rough ground, and sweeps and tickler chains which run over the seabed, creating a sediment plume to enhance catch (Clark, 2010; Jones, 1992). Trawl tows focused on hills start at the summit and move down the flank, often repeatedly following the same trawl line (Clark, 2010). Seamount fisheries are typically focused on compact and persistent fish aggregations, which leads to a high concentration of tows in a small area (Clark, 1999). This fishing pressure degrades habitat complexity with bottom trawls flattening the benthos (Althaus et al., 2009). For example, a new fishery on three seamounts off southern Australia caught $1700 \mathrm{t}$ of coral by-catch in its initial year (one third of total catch) (Anderson and Clark, 2003). This illustrates a substantial biogenic habitat modification. Corals and other deep sea invertebrates are particularly vulnerable to trawling due to their large size, immobility, longevity and fragility (Etnoyer, 2009). These invertebrates are integral to the seamount ecosystem because they increase seamount structural complexity, creating opportunities for associated species and promoting biodiversity (Etnoyer, 2009). The interaction between seamount fish species and the benthos is not well described, though it is assumed that the substantial fish aggregations present on seamounts will interact with the benthos at some point within their life cycles (Probert et al., 1997). For example, in response to disturbance orange roughy increase tilt angle and dive toward the bottom (O'Driscoll and Joux, 2012). This response might be associated with refuge from predators gained amongst complex habitat including deep-sea corals. Bottom trawls impact fish distribution in both the short term from direct catch and disturbance, and the long term, through 
habitat modification. These changes in distribution may reduce my ability to make accurate population abundance estimates of exploited stocks (from CPUE).

An effect of bottom trawling is the re-suspension of benthic sediment; this plume may assist fish capture (Jones, 1992; Martín et al., 2014) but in the deep-sea also leads to a redistribution of sediment in an environment relatively free from major sediment disruption. In fact, trawling is thought to be the major contributor to suspended sediment in the deep sea and it is proposed that re-suspension smothers the benthos (Martín et al., 2014). Trawling induced suspended sediments move down topographical features, with effects felt far beyond the site of fishing (Martín et al., 2014; Jones, 1992). Another indirect effect of bottom trawling is the disturbance related to boat and gear noise. In fresh water lakes, Jacobsen et al. (2014) demonstrate a change in fish distribution in response to noise. Large factory trawlers generate substantially more noise, and deep-sea species like orange roughy have a highly sensitive lateral line that detects vibrations (noise) (Koslow et al., 1995). Anecdotally, commercial fishers believe that fishing disturbs orange roughy, so consequently they tow nets blind (use no echo-sounder) in an effort to increase catch (M. Dunn pers. comm.). Deep-sea ecosystems generally exist in very stable conditions, and as a consequence they may be relatively severely impacted by anthropogenic disturbance (Roberts, 2002; Althaus et al., 2009). Fishing disturbance influences fish distribution, habitat, and vulnerability to capture, consequently distorting orange roughy abundance estimates. 


\subsection{Spatial Structure of Fish Populations}

Spatially structured populations complicate the relationship between population abundance and CPUE. Non-linear relationships commonly form in fisheries that target aggregations, on features or for spawning, as fishing an aggregation will produce consistently higher CPUE than an area of sparsely distributed fish. Fishermen will selectively target aggregations to improve their catch rate (Hilborn, 1985; Campbell, 2004). Therefore, as a fishery develops, the spatial distribution of both fish and fishermen varies. How fish are distributed changes the CPUE, and this spatial bias can be addressed in analysis by allocating distinct spatial strata that allow for differences in fisheries performance between locations (Walters, 2003). This spatial allocation is especially important when fishing features, such as seamounts, where fish aggregations are known to occur. Although nearly all fish stocks exhibit some form of spatial structure (Hilborn and Walters, 1987), this structure is often ignored because of poor knowledge of the fishery (Cope and Punt, 2011).

There are two commonly described non-linear relationships between population abundance and CPUE (fig.1.1). When CPUE initially declines faster than population abundance, the fishery is described as hyperdepleted. Alternatively, when CPUE remains high despite population abundance declines, the fishery is described as hyperstable. These simple relationships are suspected to occur, to some extent, in many fish stocks, especially those of deep-sea species (tab. 1.1). 


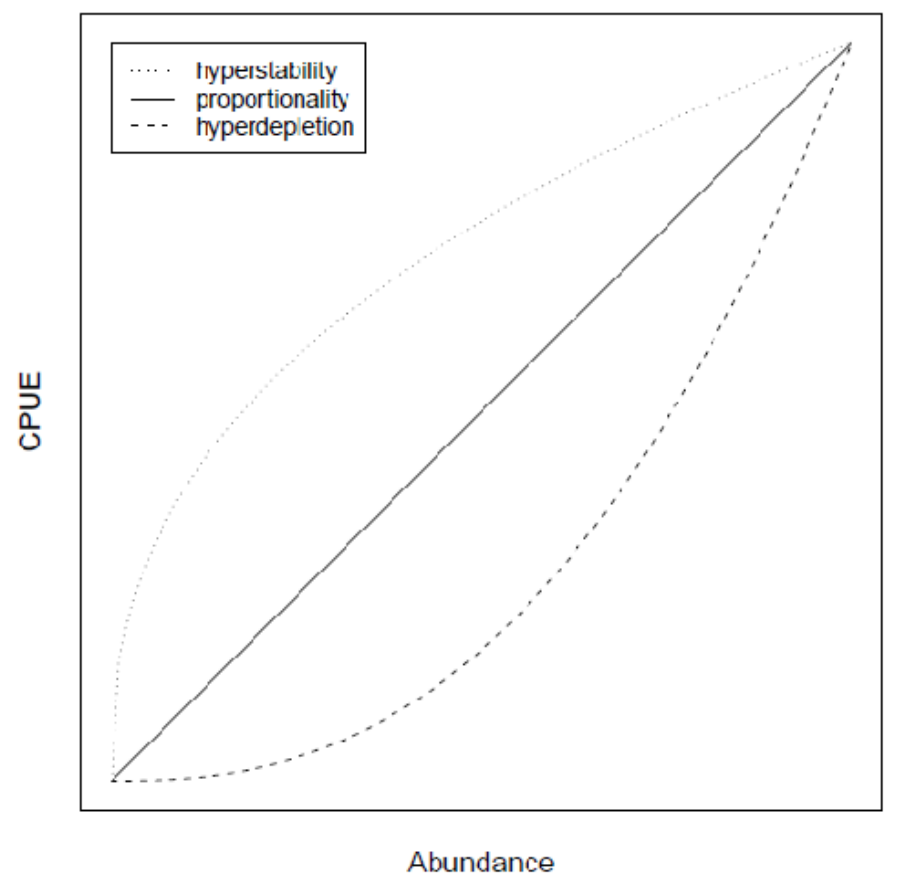

Figure 1.1: Three possible relationships between CPUE and abundance as described by Hilborn and Walters (1992)

Indicies of abundance derived from aggregations can be high, stable, and unrepresentative of the true biomass trend, which often provide the "illusion of plenty", where high levels of CPUE can be maintained while the population abundance declines (Erisman et al., 2011; Walters, 2003; Hilborn and Walters, 1992). A hyperstable relationship is thought to occur when an area is desirable. As individuals are removed from this area, they are replaced by fish from other sectors of the population, which leads to a perceived stability in population abundance, if a proportional relationship between the local CPUE and abundance is assumed. The most extreme example is where fishers target the centre of an aggregation so CPUE re- 
mains stable despite the population contracting and declining. The dangers of misinterpretation of a hyperstable relationship are illustrated in the collapse of the Northern Atlantic Cod (Gadus morhua) (Hutchings, 1996; Rose and Kulka, 1999; Rindorf and Andersen, 2008; Erisman et al., 2011). A proportional relationship between commercial CPUE and abundance was assumed for the cod fishery. Fisheries managers misinterpreted stable CPUE indices to mean a stable population abundance. While CPUE remained stable, population abundance and the area occupied by the population contracted, with a smaller area being fished each year. In a period of two years, the fishery moved from boom to bust, and reported landings dropped over 150, 000 tonnes (Canadian Department of Fisheries, 2013). Deep-sea fisheries, especially those associated with seamounts, often display what is thought to be a hyperdepletion relationship between CPUE and population abundance (fig. 1.1). Hyperdepletion is thought to be a consequence of spatially distinct fisheries where a decline in CPUE in a small spatial area is assumed to not reflect the wider population. Without proving this relationship, it is perilous to assume a local decline in CPUE does not represent the population (i.e. hyperdepletion exists), because if it does not, it will quickly become overfished and depleted. 


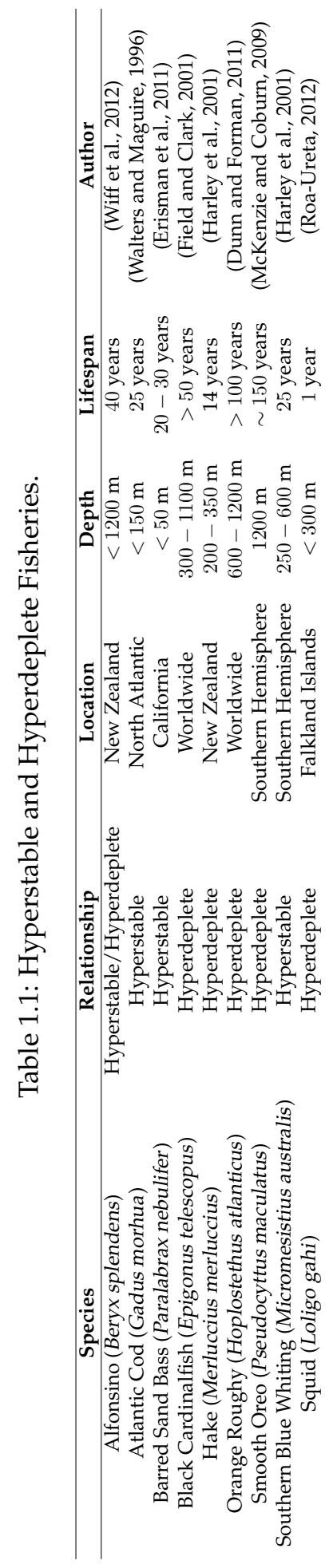


Seamounts are prominent features of the seafloor throughout the world (Clark and Rowden, 2009; Clark, 2010). They are an important component to New Zealand marine ecosystem with $\sim 800$ known features within the Exclusive Economic Zone (EEZ) (O'Driscoll and Clark, 2005). Here I define a seamount as a geographically isolated topographic feature on the seafloor taller than $100 \mathrm{~m}$, in line with Staudigel et al. (2010). Seamounts are particularly important to the fishing industry, as substantial aggregations of commercially valuable species commonly occur around and atop these loci (Tracey et al., 2004). These aggregations allow fishers to utilise short accurate tows that yield a considerable volume of catch. In New Zealand, there are major fisheries focused on seamounts that target orange roughy (Hoplostethus atlanticus), smooth oreo (Pseudocyttus maculatus), black oreo (Allocytus niger), black cardinalfish (Epigonus telescopus), bluenose (Hyperoglyphe antarctica), rubyfish (Plagiogeneion rubiginosum) and alfonsino (Beryx splendens) (Clark, 1996; Doonan et al., 2009; Clark et al., 2010; Ministry for Primary Industries, 2014). The large aggregations seen on seamounts have led to a paradigm of high productivity at these locations, which promotes these aggregations (Rowden et al., 2010). Historically, high productivity was attributed to elevated primary production above and around the seamount, and consequently elevated secondary productivity, resulting from upwelling of nutrient rich waters (Hubbs, 1958). Seamount current-topography interactions create novel flows, such as Taylor Cones, which hold the enhanced production atop the seamount (Rowden et al., 2010). This paradigm is now considered outdated, because observed seamount aggregations cannot be sustained by autochthonously derived food sources alone (Rowden et al., 2010). Most seamounts do not 
reach the photic zone, preventing any primary production (Rowden et al., 2010). Recent evidence suggests fish aggregations on seamounts are supported by horizontally and vertically advecting zooplankton, as opposed to locally enhanced production (Rowden et al., 2010; Priede et al., 2013). Fish are thought to aggregate at seamounts because of the zooplankton rich waters which provide a constant food source that can be exploited without substantial vertical or horizontal movement, which is a stark contrast to the surrounding deeper ocean.

\subsection{Orange Roughy}

Orange roughy have a worldwide distribution, with past and present fisheries off Australia, New Zealand, Namibia, Chile and Ireland (Francis and Clark, 2005), where their depth distribution is around $800-1100 \mathrm{~m}$ (Branch, 2001). The fishery in New Zealand has been by far the largest, and is the only substantial extant fishery (Dunn and Forman, 2011). Orange roughy are the dominant deep-sea species over the 20 years of Quota Management System (QMS) in New Zealand (Ministry for Primary Industries, 2014). The commercial catch of orange roughy is generally made up of individuals measuring around $35 \mathrm{~cm}$ and weighing around $2 \mathrm{~kg}$ (Ministry for Primary Industries, 2014). Orange roughy are extremely long lived, with age at maturity estimated between $25-40$ years, and a life span in excess of 100 years (Ministry for Primary Industries, 2014). As natural mortality is thought to be very low, this inherently means low productivity and leads to roughy being highly vulnerable to fishing exploitation (Clark, 
2001; Ministry for Primary Industries, 2014). Overestimated optimistic estimates of orange roughy productivity are heralded as the cause of major declines in fisheries in New Zealand and around the world (Sissenwine and Mace, 2007).

Orange roughy are a highly aggregating species, and in New Zealand they form spawning aggregations during the winter months, usually July to August (Coburn and Doonan, 1994). Some stocks are thought to undergo substantial migrations (>100km) to reach these aggregations (Pankhurst, 1988; Francis and Clark, 1998). Outside of the spawning period, orange roughy also form substantial aggregations, which are thought to be for feeding, atop of seamounts (Ministry for Primary Industries, 2014). The aggregating behaviour of orange roughy contributes to their exploitation, as high CPUE can be maintained by fishing an aggregation and seamount aggregations are fished preferentially to the surrounding areas to attain high CPUE (Clark and Dunn, 2012). There is thought to be a sequential depletion of these aggregations (Roberts, 2002), which occurs when the fleet shift fishing location to maintain a high level of CPUE (Clark et al., 2000). Sequential depletion is problematic because of a perceived stability of the CPUE index, as a consequence of the moving fleet rather than a stable fish population.

The spatial problems in orange roughy CPUE data lead to indices of abundance that may not reflect overall stock trends, although they may reflect local population trends (Clark et al., 2010). Orange roughy fisheries are characterised by steep initial declines in CPUE, which are thought to occur too quickly to reflect the wider population (hyperdepletion) (Harley et al., 2001; Francis and Clark, 2005). Therefore, the use of CPUE as an 
index of abundance is considered undesirable for orange roughy, both because of their spatially structured populations and the fishing effort targeting aggregations (Clark et al., 2010). Nevertheless, CPUE has been and is still used where fisheries independent information is unavailable or considered unreliable, for example, outside the New Zealand Exclusive Economic Zone (EEZ), and better understanding of the mechanism behind apparent CPUE hyperdepletion would greatly help management and assessment of these fisheries (Clark et al., 2010).

\subsection{Objective and Approach}

This thesis addresses the problem of apparent non-linear relationships between commercial CPUE and orange roughy population abundance. This thesis tests a range of hypotheses about orange roughy population structure in an effort to elucidate drivers of this relationship.

This project uses as a case study the largest New Zealand non-spawning orange roughy fishery, located on the Andes Complex, a collection of seamounts on the eastern Chatham Rise. This case study was chosen for the modeling component due to its long time series of CPUE data. An exploratory analysis of the Andes orange roughy fishery was undertaken, to gain a full understanding of the fishery. The CPUE is then standardised using a Generalised Linear Model (GLM), the results of which are used as a biomass index and form the basis of the next stage of the analysis. Hypotheses addressing the hyperdepletion patterns in CPUE data are developed. These hypotheses are informed from the wider literature 
and knowledge of the Andes fishery gained from the exploratory analysis. Modified Schaefer surplus production models are formed for each of the hypotheses. The hypotheses are then accepted or rejected based on comparison between their simulated output and their fit to the real historical CPUE data.

\subsection{Hypotheses for Stock Structure}

Here I describe the hypotheses considered and the rationale behind each. These hypotheses are parameterised in Chapter 2.

1. Closed Population: this hypothesis assumes there is a closed orange roughy population at the Andes Complex. The Andes population is distinct and isolated from any other areas of the Chatham Rise. As a consequence, this population is only influenced by fishing and growth at the Andes Complex with no external considerations (i.e. immigration, emigration). This is the null hypothesis and is how this fishery and many other orange roughy fisheries around the Chatham Rise have been assumed to behave (Dunn, 2006; Ministry for Primary Industries, 2014).

2. Changing Growth: this hypothesis allowed the population growth of the Andes Complex orange roughy population to change at one point over the course of the fishery. This hypothesis is motivated by concerns that large changes in population growth in New Zealand orange roughy populations may have occurred (Doonan et al., 2015). 
3. Altered Environment: This hypothesis examined the effect of the environment on two population demographics; the carrying capacity and the population growth rate. Sea surface temperature was selected as the environmental parameter because the sub-tropical convergence occurs just to the west of the Andes Complex (Dunn et al., 2009). The change in temperature over the sub-tropical convergence is around $2^{\circ} \mathrm{c}$, with the cooler temperatures of sub-Antarctic water are associated with fewer juvenile orange roughy (Dunn et al., 2009). The location of the convergence may move, and because its general location is close to the Andes, and orange roughy are thought to be influenced by temperature, there is potential for it to alter orange roughy distribution.

4. Fishing Disturbance: disturbance is hypothesised to modify orange roughy behaviour, making them less available to capture (reducing catchability). The effect of disturbance may become reduced as time passes after a disturbance event. Bottom trawling is not subtle, as nets that target roughy are large, and they create substantial vibrations, a sediment plume and remove a portion of the fish aggregation as catch. Orange roughy are known to be sensitive to disturbance, with evidence of a school moving away from a steel bar dropped over the side of a vessel (Koslow et al., 1995) and another study incidentally showed a school parting away from a submerged light (O'Driscoll and Joux, 2012). Anecdotal evidence for disturbance is provided by commercial fishers, who avoid an area after fishing it and turn off echo-sounders when setting their net, as this is also 
thought to frighten the fish (M. Dunn pers. comm.). The Namibian orange roughy fishery provides the most compelling evidence of the potential effect of disturbance, with substantial aggregations vanishing after the commencement of the commercial fishery, and this observed population change could not be accounted for by catch removals alone (McAllister and Kirchner, 2002).

5. Habitat Degradation: this hypothesis alters the carrying capacity of the Andes Complex as a consequence of changes in habitat quality, caused by bottom trawls that target orange roughy. The Andes Seamounts support an array of invertebrates which provide biogenic habitat complexity (Etnoyer, 2009). This habitat is vulnerable to the effects of bottom trawling, with as few as ten tows observed to create a significant habitat degradation (Clark, 2010), and fished seamounts observed to have half the benthic diversity of unfished mounts nearby (Koslow et al., 2001). Changes in habitat quality are long-term, with studies observing no improvement to damaged habitat over $5-10$ years (Althaus et al., 2009).

The rest of the thesis is set out as follows: In Chapter 2 the model structure and estimation methods are outlined, Chapter 3 describes an exploratory data analysis of the Andes Seamount Complex, in Chapter 4 my surplus production models are fitted and assessed, and in Chapter 5 the model results are discussed. 


\section{Chapter 2}

\section{Model Structure and Estimation Methods}

In Chapter 1, I proposed various hypotheses about the structure of the orange roughy population at the Andes. In this chapter, I give a detailed description and mathematical equations that represent the hypothesised structures, along with an explanation of the model parameters. The methodology of the generalised linear model applied in my exploratory analysis (Chapter 3) is also described.

\subsection{Surplus Production Model}

My models are based upon a Surplus Production Model (SPM). In general, a SPM considers a stock as one unit of biomass and is used to model the biomass dynamics, taking into account observed catches. The SPM has two components: the process model which describes population biomass dynamics, generating biomass estimates; and the observation model which 
relates the biomass estimates from the process model to the observed abundance index. Here I describe both models, their parameters, error structures and applications.

The process model (Eq. 2.1) and observation model (Eq. 2.2) are presented in a deterministic form, though it is possible to include a random error term in each. They combine to form the surplus production model, given by

$$
\begin{gathered}
B_{t+1}=B_{t}+f\left(B_{t}\right)-C_{t} \\
I_{t}=q B_{t}
\end{gathered}
$$

where $B_{t}$ is the biomass (tonnes) at the start of time period $t, f\left(B_{t}\right)$ is the population growth function and $C_{t}$ the catch in time $t . I_{t}$ is the estimated abundance index for time $t$, it is the product of the biomass $\left(B_{t}\right)$ for time $t$ and the catchability coefficient $q$, a constant.

The population growth function $\left(f\left(B_{t}\right)\right)$ pools recruitment, mortality and growth processes of a population into a single growth function (Eq. 2.3).

population growth $=$ new biomass $_{t}+$ biomass growth $_{t}-$ natural mortality $t$

In doing so, I are assuming the population is an undifferentiated mass, with homogenous recruitment, growth rate and natural mortality. There 
is no consideration for any population structures, e.g. age and size. The population growth function is density dependent, whereby growth is not constant but depends on the biomass;

$$
\frac{d B}{d t}=f\left(B_{t}\right)
$$

where $f^{\prime}(B) \leq 0$. Populations grow and with the absence of fishing, removals are only as a result of natural mortality. The population is part of a larger interacting ecosystem; it does not have a monopoly over the resources it requires. There are limited resources e.g. food and habitat, therefore, only a finite number of individuals can be supported. As the population approaches its maximum, the carrying capacity, there is negative feedback from the limited resources and consequently, a reduction in population growth rate. That is $f^{\prime}(B)$ tends to 0 as a population reaches carrying capacity.

\subsubsection{Schaefer Population Growth Model}

Here I utilise a Schaefer Population Growth Model that assumes logistic population growth, in the absence of fishing (Fig.2.1), for the form of $f^{\prime}(B)$; this is the simplest and most widely used of a few alternatives, for example, the Pella Tomlinson model (Hilborn and Mangel, 1997; Bolker, 2008; Haddon, 2010). The Schaefer population growth rate is given by

$$
f\left(B_{t}\right)=r B_{t}\left(1-\frac{B_{t}}{K}\right)
$$


where $r$ is the intrinsic growth rate of the population, and $K$ is the population's carrying capacity (Schaefer, 1954).

The Schaefer growth rate is density dependent and is determined by the proportion of biomass to carrying capacity $\left(B_{t} / K\right)$. The growth rate is symmetrical around $K / 2$, the biomass that gives the maximum population growth rate (Fig. 2.1). Minimum growth rates are reached at the asymptotes 0 (no population) and $K$ (carrying capacity), evident in figure 2.1.

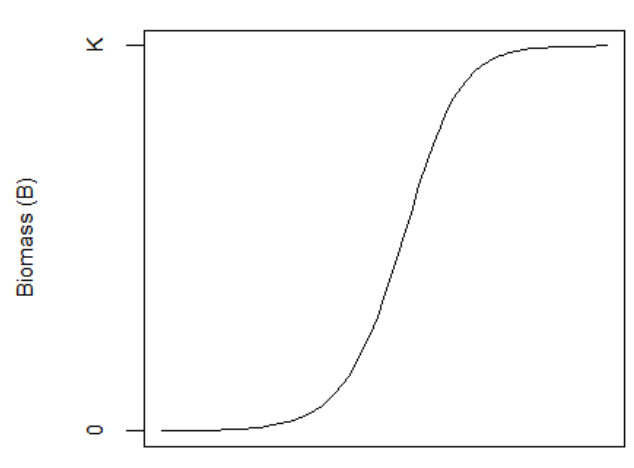

Time (t)

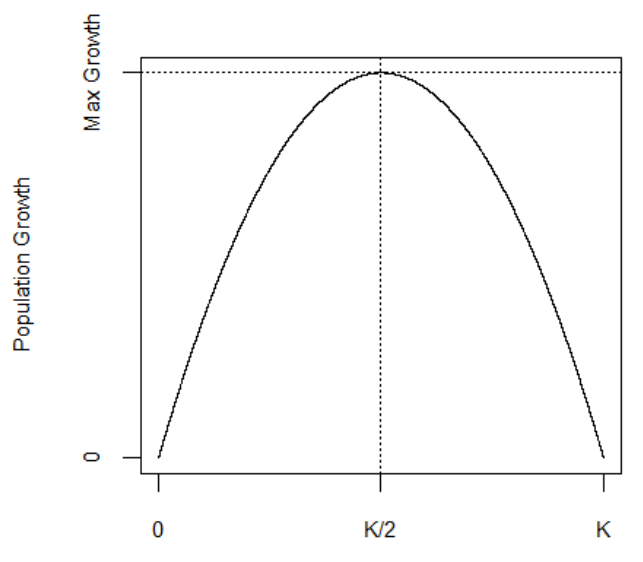

Biomass (B)

Figure 2.1: Logistic growth curve of the Schaefer model (left); and population growth $\left(r B_{t}\left(1-\frac{B_{t}}{K}\right)\right)$ as biomass increases (right).

Surplus production is the annual growth in biomass $r B_{t}\left(1-\frac{B_{t}}{K}\right)$, which can be removed by catch whilst the biomass $B_{t}$, stays constant. Fisheries managers endeavour to maximise surplus production to enable maximum catch. Surplus production occurs when the biomass is lower than carrying capacity $(B<K)$. Because of the symmetry in the logistic growth, 
curve maximum production is $r K / 4$, at $B_{t}=K / 2$. This point is known as the Maximum Sustainable Yield (MSY) and is defined as the highest theoretical yield that can be continuously taken from a stock under existing environmental conditions without significantly affecting the reproduction process (Tab. 1).

The Schaefer SPM has been extensively described and its application is the subject of various fisheries texts (Hilborn and Walters, 1992; Hilborn and Mangel, 1997; Haddon, 2010). SPM's are widely used for catch-perunit-effort data, especially when there are no other populations characteristics available, for example, age structure (Punt, 2003). The underlying assumption in such applications is that catch-per-unit-effort $\left(C_{t} / E_{t}\right)$ is proportional to the population abundance index $\left(I_{t}\right.$, Eq. 2.2).

The process model with a Schaefer growth function (Eq. 2.6) simplifies the population demographics into two parameters, $r$ and $K$, that determine annual population change. In this thesis, I assume that the Andes Complex fishery was pristine, unfished, prior to the start of my dataset and therefore the population was at carrying capacity at the start of the fishery $\left(B_{1991}=K\right)$. The basic Schaefer model assumes that $r$ and $K$ are constants and form a single closed population with no emigration or immigration. The process model is given by,

$$
B_{t+1}=B_{t}+r B_{t}\left(1-\frac{B_{t}}{K}\right)-C_{t}
$$

My time series is annual, so biomass next year is determined by the 
biomass this year plus population growth, and less removals from catch this year. Population growth is composed of annual growth of the biomass, recruitment and less natural mortality. Population growth is density dependent based on the fraction of the carrying capacity made up by the current biomass.

In summary the assumptions of my Surplus Production Model are:

- parameters $r, K, q$ are constant;

- $r$ is constant irrespective of age composition and size composition of the population;

- the Andes Complex orange roughy population is a closed single stock (no emigration or immigration);

- the catch data for the Andes Complex orange roughy fishery are accurate;

- fishing mortality and growth take place simultaneously;

- catch-per-unit-effort is proportional to biomass;

The Schaefer SPM is simple, free of vigorous data needs of alternate models (e.g. age structures). A SPM is appropriate here, given the use of CPUE data and the lack of other population information available. There has been similar applications with rattails (Large, 2013). A SPM provides a simple base model to build on or restructure as required for my model hypotheses. 


\subsubsection{Model Equations}

The hypotheses behind each of these models have been introduced in Chapter 1, and here I specify their form and any salient parameters.

\section{Closed Population Model}

My first model is a closed population of orange roughy at the Andes Complex. This model is a standard Schaefer model

$$
B_{t+1}=B_{t}+r B_{t}\left(1-\frac{B_{t}}{K}\right)-C_{t}
$$

\section{Changing Growth Model}

This model allows the intrinsic growth rate of the population to change at one point in the fisheries time series (Eq. 2.8).

$$
\begin{gathered}
B_{t+1}=B_{t}+r B_{t}\left(1-\frac{B_{t}}{K}\right)-C_{t} \\
r=\left\{\begin{array}{cl}
r_{1} & \left(t \leq t^{*}\right) \\
r_{2} & \left(t>t^{*}\right)
\end{array}\right.
\end{gathered}
$$

where $t^{*}$ is the transition point between the intrinsic growth rates $\left(r_{1}, r_{2}\right)$

\section{The Altered Environment Model $(r)$}

The Altered Environment Model ( $r$ ) allows for the population growth rate to change annually based on an observed change in environmental conditions (Eq. 2.9). I chose Sea Surface Temperature (SST) for the Andes region 
$\left(43.5-44.5^{\circ} S, 175.5-174.5^{\circ} \mathrm{W}\right)$ as an indicator of environmental conditions. SST was obtained by averaging monthly mean data, from 1991 2013, to form annual SST values. The data were obtained from the National Oceanic and Atmosphere Administration (NOAA) (http: / / www . esrl.noaa.gov/psd/data/gridded/data.noaa.oisst.v2.html).

$$
\begin{aligned}
& B_{t+1}=B_{t}+r_{t} B_{t}\left(1-\frac{B_{t}}{K}\right)-C_{t} \\
& r_{t}=m S S T_{t}+c
\end{aligned}
$$

where the growth rate $r_{t}$ in time $t$ is assumed to be linearly related to annual mean SST. This relationship is dictated by $m$ the slope and $c$ the intercept.

\section{The Altered Environment Model $(K)$}

The Altered Environment Model $(K)$ allows for the population carrying capacity to change annually, based on an observed change in environmental conditions (Eq. 2.10). This is equivalent to Altered Environment Model $(r)$ but the environmental vector is applied to $K$ instead of $r$.

$$
\begin{aligned}
& B_{t+1}=B_{t}+r B_{t}+r B_{t}\left(1-\frac{B_{t}}{K_{t}}\right)-C_{t} \\
& K_{t}=m S S T_{t}+c
\end{aligned}
$$

where the growth rate $K_{t}$ in time $t$ is assumed to be linearly related to annual mean $S S T$. This relationship is dictated by $m$ the slope and $c$ the intercept. 


\section{The Disturbance Model}

Disturbance alters the catchability $q$, of orange roughy. The Disturbance Model incorporates information about the number of tows $n_{t}$, as a metric for the level of disturbance (eq. 2.11).

$$
\begin{aligned}
B_{t+1} & =B_{t}+r B_{t}\left(1-\frac{B_{t}}{K}\right)-C_{t} \\
I_{t} & =q_{t} B_{t} \\
q_{t} & =\frac{q}{\left(n_{t-2} / 4\right)+n_{t-1} / 2+n_{t}}
\end{aligned}
$$

where the population catchability $q_{t}$ in time $t$ is scaled by the cumulative number of tows in the last three years $(t-2, t-1, t)$. There is a decay in the disturbance of tows over time, with tows in the past having an exponentially decaying contribution to disturbance.

\section{The Habitat Degradation Model}

The Habitat Degradation Model takes into account the area of habitat affected by fishing activity $(H D$, Eq. 2.12). A reduction in habitat quality scales the carrying capacity, reducing the biomass of fish supported.

$$
B_{t+1}=B_{t}+r B_{t}\left(1-\frac{B_{t}}{K / H D_{t}}\right)-C_{t}
$$

where $H D_{t}$ is the cumulative habitat damage in time $t$

The Habitat Degradation Model takes into account the area of the Andes Complex affected by fishing activity ( $H D$, Eq. 2.12). The Andes Complex was overlaid with a grid, as shown in figure 2.2. Clark and Tittensor (2010) showed that 10 tows was the threshold for a significant reduction in 
coral cover. Grid cells were allocated a 0 for habitat damage if they were unfished, a 1 if they sustained $<10$ tows and a 2 if they sustained $\geq 10$ tows. The values of habitat damage were assessed annually for each grid cell, and the effect of habitat damage was considered cumulative, as once the habitat was degraded it did not recover in the timescale of the fishery. Therefore, once a grid cell attained a score of 2 (degraded) it retained this value.

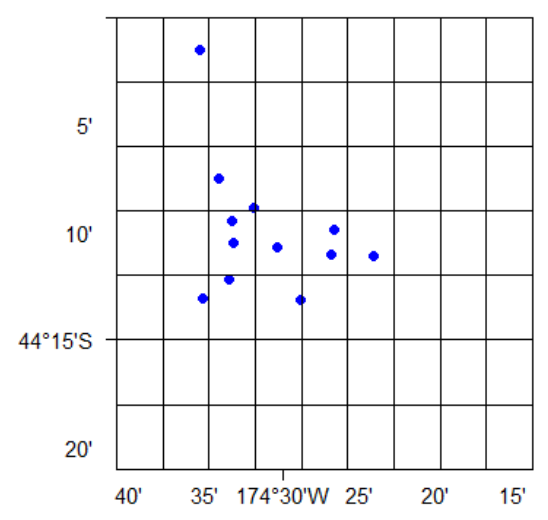

Figure 2.2: The grid for the Andes Complex used to create the index of habitat damaged $(H D)$; each grid cell is $0.05 \times 0.05$ degree latitude longitude bins. Tows were allocated to grid cells based on the tow starting co-ordinates. The dots represent the peak of the each of the Andes Complex seamounts.

Three habitat models were considered; the first model was an Unweighted Habitat Degradation Model, where the value of the habitat was considered homogenous over the the Andes area. The second model was a Transformed Habitat Degradation Model, which is the same as the first model, but the habitat damage vector was log-transformed. The final 
model was the Weighted Habitat Degradation Model, which gave grid cells that included seamounts an order of magnitude higher weighting, as orange roughy have been observed at much higher densities on seamounts than the surrounding area (Dunn and Forman, 2011).

\subsubsection{Model Errors}

Errors are associated with both the process $\left(e^{\epsilon_{t}^{\nu}}\right.$ (eq. 2.13)) and observational models ( $e^{\epsilon_{t}^{u}}$ (Eq. 2.14)). It is commonly assumed that these errors follow a Normal Distribution (Bolker, 2008; Haddon, 2010). When errors are incorporated the SPM can be written as

$$
\begin{aligned}
B_{t+1} & =\left(B_{t}+r B_{t}\left(1-\frac{B_{t}}{K}-C_{t}\right) e^{\epsilon_{t}^{\nu}}\right. \\
I_{t} & =q B_{t} e^{\epsilon_{t}^{u}}
\end{aligned}
$$

where

$$
\epsilon_{t}^{\nu} \stackrel{\mathrm{iid}}{\sim} N\left(0, \sigma_{\nu}^{2}\right), \quad \epsilon_{t}^{u} \stackrel{\mathrm{iid}}{\sim} N\left(0, \sigma_{u}^{2}\right)
$$

Considering the observational errors first, $\epsilon_{t}^{u}$ has the density function:

$$
f\left(\epsilon_{t}^{u}\right)=\frac{1}{\sqrt{2 \pi \sigma_{u}^{2}}} \exp \left(-\frac{1}{2 \sigma_{u}^{2}}\left(\epsilon_{t}^{u}\right)^{2}\right)
$$


To determine the density function for $I_{t}$, I consider $I_{t}$ as a function of $h($.$) of \epsilon_{t}^{u}$. That is, I let

$$
I_{t}=h\left(\epsilon_{t}^{u}\right), \quad \text { where } \quad h\left(\epsilon_{t}^{u}\right)=q B_{t} e^{\epsilon_{t}^{u}}
$$

The inverse of $h($.$) is given by$

$$
h^{-1}\left(I_{t}\right)=\epsilon_{t}^{u}
$$

where from equation 2.14 I have

$$
h^{-1}\left(I_{t}\right)=\epsilon_{t}^{u}=\log \left(I_{t}\right)-\log \left(q B_{t}\right)
$$

Then, using the change of variable theorem, the probability density function $g\left(I_{t}\right)$ is specified by the relation

$$
\begin{aligned}
g\left(I_{t}\right) & =f\left[h^{-1}\left(I_{t}\right)\right]\left|\frac{d h^{-1}\left(I_{t}\right)}{d I_{t}}\right| \\
& =\frac{1}{\sqrt{2 \pi \sigma_{u}^{2}}} \exp \left(-\frac{1}{2 \sigma_{u}^{2}}\left[\log \left(I_{t}\right)-\log \left(q B_{t}\right)\right]^{2}\right)\left|\frac{1}{I_{t}}\right| \\
& =\frac{1}{I_{t} \sqrt{2 \pi \sigma_{u}^{2}}} \exp \left(-\frac{1}{2 \sigma_{u}^{2}}\left[\log \left(I_{t}\right)-\log \left(q B_{t}\right)\right]^{2}\right)
\end{aligned}
$$

This is the density function for a log-Normal random variable, therefore

$$
\log \left(I_{t}\right) \sim N\left(\log \left[q B_{t}\right], \sigma_{u}^{2}\right)
$$


The process errors, $\epsilon_{t}^{\nu}$ have the density function;

$$
f\left(\epsilon_{t}^{\nu}\right)=\frac{1}{\sqrt{2 \pi \sigma_{\nu}^{2}}} \exp \left(-\frac{1}{2 \sigma_{\nu}^{2}}\left(\epsilon_{t}^{\nu}\right)^{2}\right)
$$

and the probability density function $g($.$) for the biological process is$ generated in the same manner which gives:

$$
g\left(B_{t+1}\right)=\frac{1}{B_{t+1} \sqrt{2 \pi \sigma_{\nu}^{2}}} \exp \left(-\frac{1}{2 \sigma_{\nu}^{2}}\left[\log \left(\frac{B_{t+1}}{B_{t}+r B_{t}\left(1-\frac{B_{t}}{K}\right)-C_{t}}\right)\right]^{2}\right)
$$

and, therefore

$$
\log \left(B_{t+1}\right) \sim N\left(\log \left[B_{t}+r B_{t}\left(1-\frac{B_{t}}{K}\right)-C_{t}\right], \sigma_{\nu}^{2}\right)
$$

There will be observational error as a result of the catch data being visually estimated on board. Each skipper will have a individual bias in these catch estimates. Observational errors can also occur, due to varied catchability $q$, of the orange roughy. Catchability is likely to vary, as a consequence of altered fish or fisher behavior (Wilberg et al., 2009). Process errors can occur, for example, as a consequence of age structure; something my SPM does not consider. Alternatively, if any of the demographics influencing population growth vary through time, then further process error will be incurred (Punt, 2003). Process error models generally lack precision in their parameter estimates, and they also can create a bias in observation error models (Punt, 2003), so consequently I will utilise an observation error only model. Both process and observational errors should ideally be considered when fitting the SPM. However, fitting both errors 
in the model can be problematic (Haddon, 2010) and many studies opt to only include observational errors (Hilborn and Walters, 1992; Haddon, 2010).

The unknown parameters in my SPM require estimation. I utilize maximum likelihood estimation, including the error term in the observational model, but assuming a deterministic process model.

\subsection{Maximum Likelihood Estimation}

Each of my hypothesised surplus production models has a set of unknown parameters that requires estimation. Maximum likelihood estimation determines the best estimates for the values of the parameters that maximise the likelihood of the observed data. I are using an observation error only model, so process errors are assumed to be deterministic $\left(\sigma_{\nu}^{2}=0\right)$ and observational errors account for all the variation in observed abundance index. The likelihood of the observed abundance index, $I_{t}$, from equation 2.2 given the biomass, $B_{t}$, is:

$$
\begin{aligned}
L\left(q, B_{1}, r, K, \sigma_{u} \mid I_{t}\right) & =g\left(I_{t} ; q, B_{1}, r, K, \sigma_{u}\right) \\
& =\frac{1}{I_{t} \sqrt{2 \pi \sigma_{u}^{2}}} \exp \left(-\frac{1}{2 \sigma_{u}^{2}}\left[\log \left(I_{t}\right)-\log \left(q B_{t}\right)\right]^{2}\right)
\end{aligned}
$$

The probability of seeing my data given the parameter estimates, $P\left(I_{t} \mid\right.$ $\left.q, r, K, B_{t}, \sigma_{u}^{2}\right)$, is equivalent to the likelihood of the parameter estimates given the data $L\left(q, B_{1}, r, K, \sigma_{u} \mid I_{t}\right)$. I utilised maximum likelihood estimation, that is, were looking for a set of parameter values that maximise the likelihood. The maximum likelihood is defined as 


$$
\arg \max _{q, B_{1}, r, K, \sigma_{u}} L\left(q, B_{1}, r, K, \sigma_{u} \mid I_{t}\right)
$$

or equivalently the minimum log-likelihood

$$
\arg \min _{q, B_{1}, r, K, \sigma_{u}}-\log L\left(q, B_{1}, r, K, \sigma_{u} \mid I_{t}\right)
$$

The joint density of the sample makes the implicit assumption of conditional independence between observations from different years. This assumption is likely to be violated, based on the surplus production model biomass $B_{t+1}$ in time $t+1$, is dependent on the biomass $B_{t}$ in time $t$ (eq. 2.1). The full likelihood function for the observational model is:

$$
L\left(K, r, q, B_{1}, \sigma_{u} \mid I_{t}, C_{t}\right)=\prod_{t=1}^{n} \frac{1}{I_{t} \sqrt{2 \pi \sigma_{u}^{2}}} \exp \left(\frac{\left[\log \left(I_{t}\right)-\log \left(q B_{t}\right)\right]^{2}}{2 \sigma_{u}^{2}}\right)
$$

where $n$ is the total number of years and, for the basic Schaeffer logistic growth model,

$$
B_{t+1}=B_{t}+r B_{t}\left(1-\frac{B_{t}}{K}\right)-C_{t}
$$

The negative log-likelihood equals 


$$
\begin{aligned}
& -l\left(K, r, q, B_{1}, \sigma_{u}^{2} \mid C_{t}, I_{t}\right) \\
= & -\log \left[\prod_{t=1}^{n} \frac{1}{I_{t} \sqrt{2 \pi \sigma_{u}^{2}}} \exp \left(\frac{\left[\log \left(I_{t}\right)-\log \left(q B_{t}\right)\right]^{2}}{2 \sigma_{u}^{2}}\right)\right] \\
= & -\sum_{t=1}^{n} \log \left[\prod_{t=1}^{n} \frac{1}{I_{t} \sqrt{2 \pi \sigma_{u}^{2}}} \exp \left(\frac{\left[\log \left(I_{t}\right)-\log \left(q B_{t}\right)\right]^{2}}{2 \sigma_{u}^{2}}\right)\right] \\
= & \sum_{t=1}^{n} \log \left(I_{t}\right)+\frac{n}{2} \log (2 \pi)+\frac{n}{2} \log \left(\sigma_{u}^{2}\right)+\sum_{t=1}^{n} \frac{\left[\log \left(I_{t}\right)-\log \left(q B_{t}\right)\right]^{2}}{2 \sigma_{u}^{2}} \\
= & \frac{n}{2} \log \left(\sigma_{u}^{2}\right)+\sum_{t=1}^{n} \frac{\left[\log \left(I_{t}\right)-\log \left(q B_{t}\right)\right]^{2}}{2 \sigma_{u}^{2}}
\end{aligned}
$$

As the first terms of 2.30 are constant, they can be removed for simplification to give equation 2.31 .

\subsection{Parameter Estimation}

I assume that $\left(B_{1}=K\right)$ which leaves the unknown parameters $\left(r, K, q, \sigma_{u}\right)$ to estimate using Maximum Likelihood Estimation (MLE). I use optim function in the statistical software R (R Core Team, 2015)(Example R code for this process is in Appendix 6.1). I have four unknown parameters for optim to estimate. With four unknown parameters $(k=4)$, the loglikelihood surface is in 4 dimensional space. The MLE is the vector of values at the minimum of the negative log-likelihood surface.

I used optim to search for this minimum. Optim has a range of optimisation methods available. Here I used the "L-BFGS-B" method, which is a quasi-Newton method. Quasi-Newton methods calculate derivatives to 
find the slope of the log-likelihood surface; where the slope is zero, a minimum has been reached (R Core Team, 2015). The "L-BFGS-B" method allows constraints to be placed on the upper and lower limits of parameter values. This feature was used to keep the algorithm in realistic parameter space. The other optimisation method used in this analysis was simulated annealing "SANN" (a Metropolis algorithm), which is a stochastic global optimizer that randomly selects a candidate point to move to, then accepts or rejects this position based on an acceptance probability that depends on the likelihood (Bolker, 2008). Simulated annealing was used when it was suspected the algorithm had converged to a local minimum, as opposed to the desired global minimum (Bolker, 2008). Opt im is widely acknowledged to be a temperamental function (Nash and Dalzell, 2013; Bolker, 2008), especially when working within constraints like the "L-BFGS-B" method. It provides warnings related to flat gradients and maximum iterations, but more subtle problems are difficult to detect. Optimx is a new reworked version of optim (Nash and Varadhan, 2011). The main improvement relevant to this work is that optimx provides an explanation of convergence failures. Optimx was used when suspected convergence problems were encountered, but no material difference in performance was detected between optim and optimx.

Starting values and parameter bounds are important considerations when applying the optim function (Bolker, 2008). Starting values were selected based on prior studies of the fishery at the Andes Complex and biological knowledge about orange roughy. C++ Algorithmic Stock Assessment Laboratory (CASAL) model runs for the Andes Complex esti- 
mated $B_{0} \sim 28000$ tonnes (Dunn, 2006), which was used as my starting value for $K$. The initial bounds around $K$ were set as the maximum catch for the lower bound and 56000 tonnes for the upper bound (double the CASAL estimate). Orange roughy are very slow growing (Francis and Clark, 2005), so $r$ was set at 0.15 with an initial lower bound at 0.01 and upper bound at 0.5 . The catchability coefficient is an unknown constant, so I set the starting value at 0.1 and allowed $q$ to range between 0.00001 and 1, the latter because it is unrealistic if more than $100 \%$ of the stock was be taken annually. The variance $\sigma_{u}^{2}$ was bounded between 0.00001 and 1 . When parameters in the SPM were adjusted based on my hypotheses, parameter bounds were modified as required to enable convergence. This part of the investigation was treated purely statistically, and the assessment of the biologically credibility of final values will be discussed in Chapter 4.

Therefore the MLE procedure is as follows:

1. Input the observed index of abundance $I_{t}$ and catch $C_{t}$

2. Input starting values for the unknown parameters $\left(K, r, q, \sigma_{u}\right)$

3. Compute estimates of $B_{t}$ using equation 2.1

4. Compute estimates of $I_{t}$ using equation 2.2

5. Calculate negative log-Likelihood using equation 2.31

6. Repeat steps $3-5$ for all $t$ and sum - $\log \mathrm{L}$ over $t$ to find the overall likelihood 
7. Repeat steps $2-6$ until confident algorithm has converged at a global minimum

\subsection{Generalised Linear Models}

The model analysis component of this thesis requires a standardised abundance index for the Andes Complex to compare SPM model fits to. I wanted to use CPUE as an index of abundance but the catch rate records from the commercial fleet were biased by exogenous information that had to be removed to form my final Andes Complex orange roughy abundance index. The catches were standardised to account for these biases. Generalised Linear Models (GLM) are the most commonly used methods to standardise catch and effort data (Maunder and Punt, 2004). The central assumption of a generalised linear model is that there is a linear relationship between the predictor variable(s) and a function of the mean of the response variable, given by:

$$
g\left(\mu_{i}\right)=x_{i}^{T} \beta
$$

where $g$ is a differentiable and monotonic link function, $\mu_{i}=E\left(Y_{i}\right), Y_{i}$ is the response variable for observation $i, x_{i}$ is a vector of predictor variables and $\beta$ is a vector of model coefficients (Maunder and Punt, 2004).

Here I apply a delta two-step GLM to incorporate the information about zero catches into my abundance estimates. The delta method is the best practice for catch and effort data (Campbell, 2015) because the distribution 
of the catch is generally log-normal, with an excess of zeros. That is, there is a skew to the right in the probability density, due to tows that fail to catch orange roughy (Fig. 2.3).

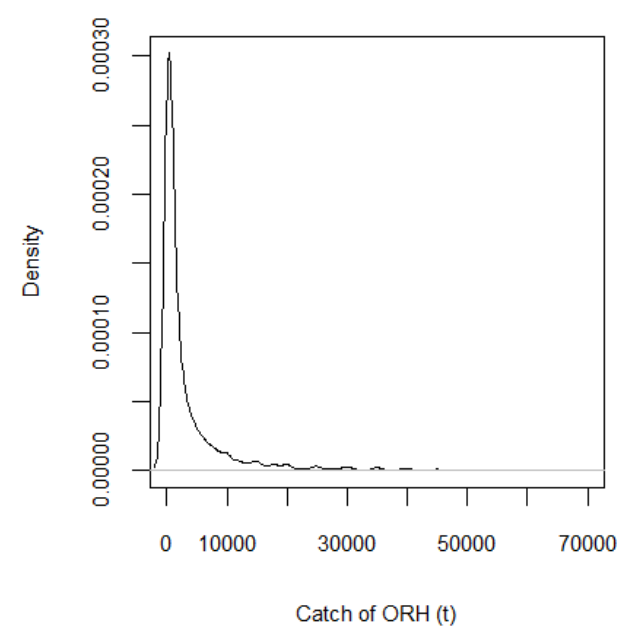

Figure 2.3: The probability density curve for the catch data.

$$
\operatorname{Pr}(Y=y)= \begin{cases}w, & \text { if } y=0 \\ (1-w) f(y), & \text { otherwise }\end{cases}
$$

where $y$ is an observed orange roughy catch rate, $w$ is the probability of a 0 observation, $1-w$ is the probability of capture $(\neq 0)$ and $f(y)$ the mean of the log-normal distribution of catch.

The delta method models this in two steps: a model of the probability of obtaining a zero catch rate and a model of the catch rate given that the catch is non zero (Eq. 2.33). The first model examines a binary random 
variable which is zero if no orange roughy were captured and unity otherwise. It has a Bernuolli distribution with a probability of a zero catch $w$, and I will model this using a binomial GLM. The second model has a catch (tons per tow) response variable given that catch is non-zero. I will model this by log-transforming the response variable and applying a Gaussian GLM. The delta method captures information about the distribution of orange roughy in the binomial GLM and abundance of orange roughy in the gaussian GLM. The final delta method abundance index is the product of the binomial and gaussian model predictions.

Confidence intervals of the final delta abundance index estimate were obtained using a non-parametric bootstrap approach. Bootstrap methods are used because there is no analytical solution for the precision of the abundance index formed from a delta approach. The assumptions about the error structures of these distributions are checked, an observed vs expected plot is used for the binomial GLM and a quantile-quantile plot for the lognormal GLM. 
42 CHAPTER 2. MODEL STRUCTURE AND ESTIMATION METHODS 


\section{Chapter 3}

\section{Exploratory Analysis of the Andes}

\section{Fishery}

The New Zealand orange roughy fishery is centered around the Chatham Rise (CR). The Chatham Rise is hypothesised to have two orange roughy stocks (Dunn and Devine, 2010); the larger is on the eastern and southern flank (fig. 3.1) and the smaller is west of $178^{\circ} \mathrm{W}$. This thesis focuses on one of the distinct sub-stock fisheries, located at the Andes Seamount Complex on the eastern CR. This is the most data rich of the seamount fisheries, within the larger of the CR orange roughy populations. Chatham Rise seamount fisheries are characterised by a initial sharp reduction in their orange roughy abundance indices, which is a poor fit to the index in traditional biomass models (Clark et al., 2010). It has been suggested that this poor fit is a consequence of a hyperdepletion relationship between CPUE and population abundance (Hicks, 2013). 


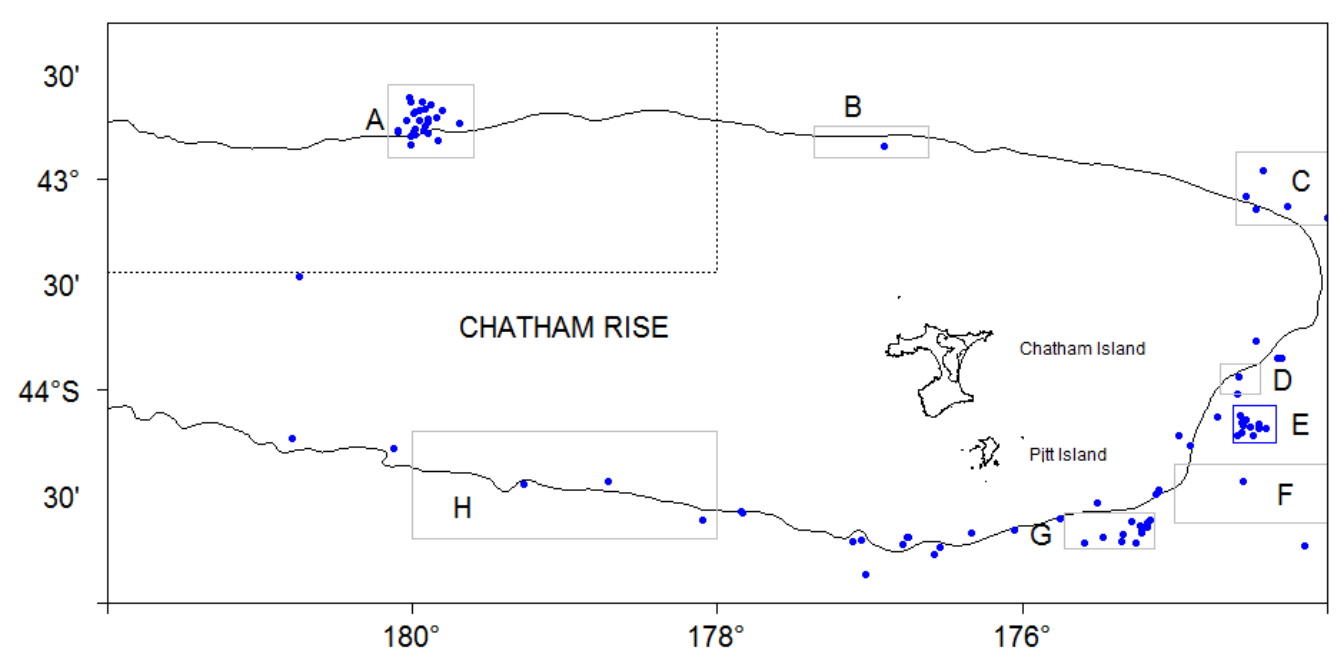

Figure 3.1: The Eastern Chatham Rise orange roughy fishery; composed of eight distinct areas, A: the Graveyard Complex B: The Spawning Box, C: The Northeast Hills, D: Kenwood, E: The Andes Complex, F: The Middleground, G: Chiefs \& Neighbours and H: Hedgerville as defined in Anderson \& Dunn 2012. Seamount features are plotted with blue dots marking their peaks, the proposed population boundary between the two Chatham orange roughy populations from Dunn \& Devine (2010) is shown by the dashed line, and the $1000 \mathrm{~m}$ depth contour has been added.

\subsection{Andes Complex}

The Andes Complex is a group of 11 hills, located within $185^{\circ} 32^{\prime}-185^{\circ} 77^{\prime}$ W $44^{\circ} 00^{\prime} 44^{\circ} 35^{\prime} \mathrm{S}$, on the south-east flank of the Chatham Rise. The hills are closely spaced, with a maximum distance between peaks of $25 \mathrm{~km}$. Seamount peaks range from 644 to $1008 \mathrm{~m}$ in depth, making them a viable option for a seamount trawl fishery. A significant feature of Andes Seamounts is a large continuous series of hills on the western fringe (Fig. 3.2). There is a single large solitary hill (Jimmy) to the west of this feature and the remainder of the complex is composed of a series of distinct smaller hills, all of which have been named by the fishing industry (Fig. 
3.2). The catch composition from the Andes Complex is spatially homogeneous, and orange roughy, smooth oreo and black oreo are the dominant species (Tracey et al., 2004). A commercial bottom-trawl fishery targeting orange roughy started on the Andes in 1989 and is still extant today (Ministry for Primary Industries, 2014). The Andes roughy fishery targets fish aggregations, which are thought to be feeding fish.

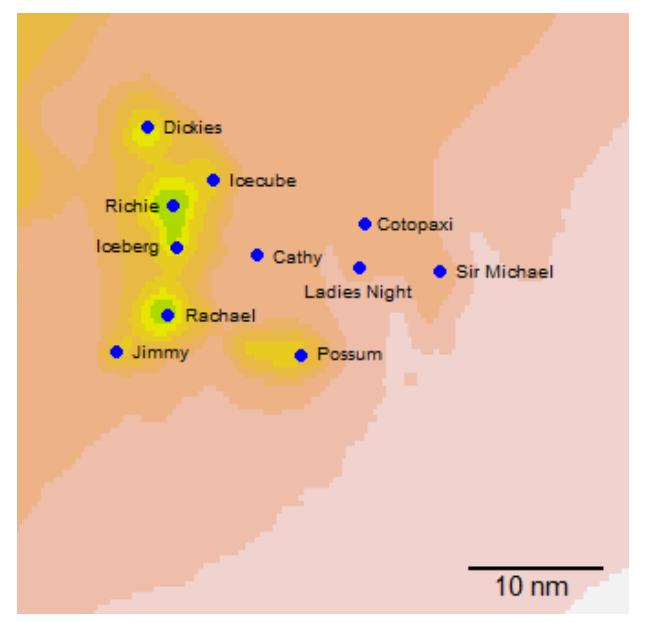

Figure 3.2: The Andes Complex; coloured bathymetry indicates depth (from $600 \mathrm{~m}$ to $2000 \mathrm{~m})$; hill peaks are indicated by blue dots, hill names are adjacent to peaks $(10 \mathrm{~nm}=$ 10 nautical miles).

\subsection{Analysis}

This chapter describes an exploratory analysis of the Andes orange roughy fishery up until 2013. The data utilised are catch records from commercial fishing vessels provided by the Ministry for Primary Industries (MPI). All commercial fishing vessels in New Zealand waters are required to fill out catch records, and these data are provided on commercial Trawl Catch Ef- 
fort Processing Return (TCEPR) forms. For each tow the following information was recorded: vessel id, vessel tonnage, kilowatts of vessel power, and for each tow the start date, start time, start position, target species, depth, speed, end date, end time, end position, and catch of orange roughy in kilograms. Most of the records were complete, with the exception of tows pre-dating 1990 which did not have an end position.

Visual displays of the location of historic tows were undertaken to elucidate any patterns to the fishing activity. The start and end positions of all tows in the Andes Complex were plotted using the program $\mathrm{R}$ ( $\mathrm{R}$ Core Team, 2015) and package nzPlot (Knowles et al., 2012). A bathymetric map of the seamount complex was obtained from NIWA's OS 2020-seismicand-multibeam project (MacKay et al., 2005) and added to the maps to provide some auxiliary information about feature orientation. Hill peaks were plotted to allow the interpretation of the feature targeted by each tow. Prior to 2004, tow start and end coordinates were generally rounded to the nearest 10 minutes. This resulted in tows being plotted over the top of each other.

\subsection{History of the Andes Fishery}

The first reported commercial fishing for orange roughy at the Andes Complex was in 1989. This commenced an exploratory phase lasting two years, with Possum, Cotopaxi and Sir Michael seamounts mostly targeted. Excluding Cathy, all the hills of the Andes have been subject to fishing pres- 
sure, with some evidence of sequential depletion. The north of the large Andes feature (Dickies, Icecube, Richie \& Iceberg) was not targeted by the fleet until 1992, and the southern end (Rachael \& Jimmy) was first fished in 1996 (fig. 3.2).

From 1991, a large scale orange roughy fishery was underway. The Andes, like many other seamount fisheries, was characterised by a distinct "boom" and "bust" cycle at the start of the fishery (Clark and Rowden, 2009). The boom commenced in 1990, with average CPUE peaking in 1992 at 10.12 tonnes per tow (fig. 3.3). The CPUE metric tonnes per tow was used because this seamount fishery utilised exclusively short tows, as a consequence, differences in tow durations are negligible. After 1992, there was a substantial decline in catch, as a consequence of a reduction in both CPUE and effort. However, the decline in CPUE halted in 1995, where it remained relatively stable at $2 \_3$ tonnes per tow, until 2002. Over the same time period, fishing effort remained constant, around 500 tows per year, until 2003, when there was a substantial increase. CPUE slowly declined, eventually plateauing in 2009 at less then one tonne per tow. Since 2007, there was a reduction in fishing effort in the Andes region and since 2009, CPUE has risen. The cumulative catch extracted from the Andes increased rapidly during the boom phase of the fishery, and the annual catch was relatively stable thereafter. In 2008 , the catch trajectory flattened due to annual catch declines, as a consequence of a decline in effort (Fig. 3.3). 

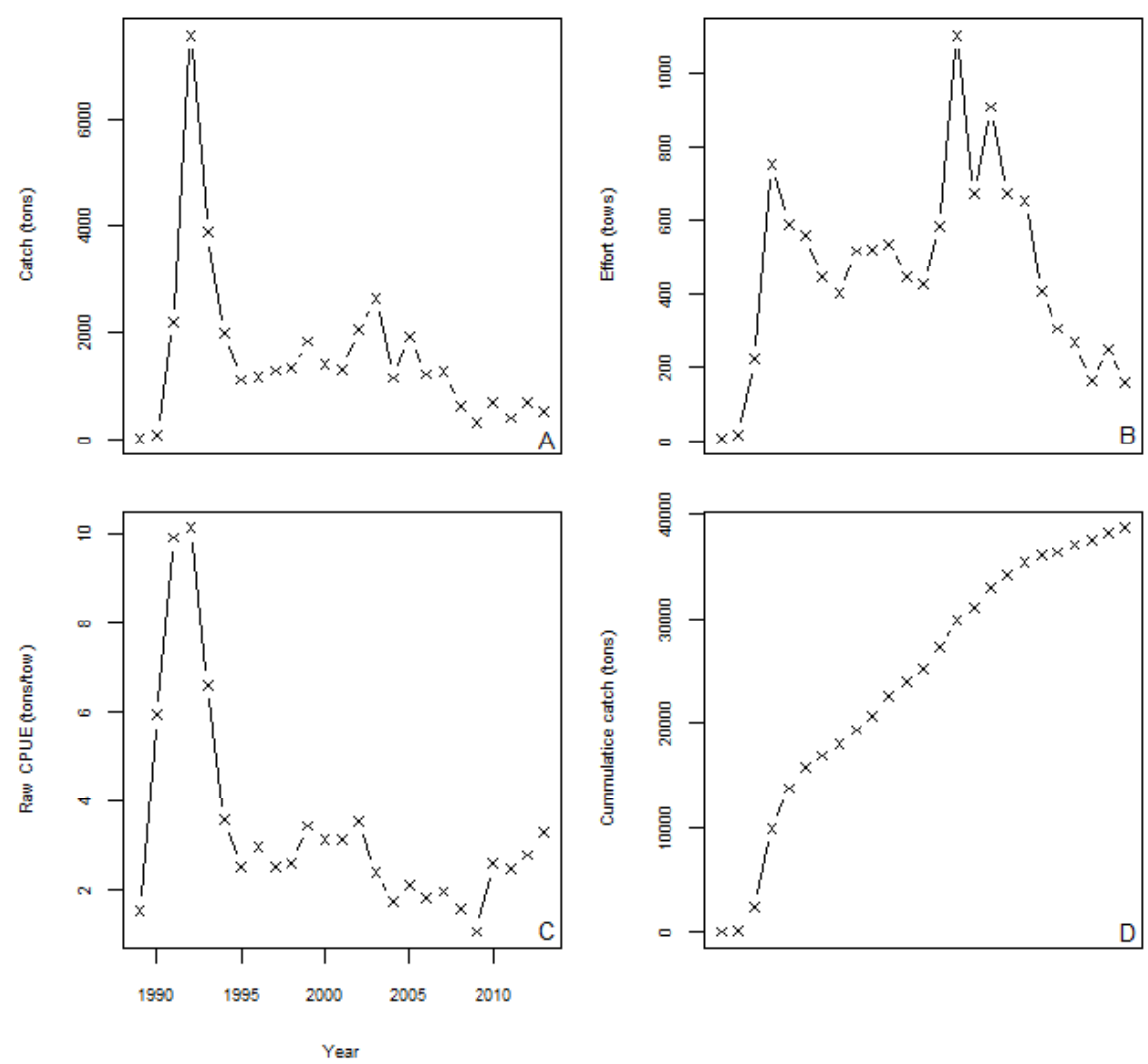

Figure 3.3: History of the Andes Seamount Complex orange roughy fishery from 1989 to 2013. A, Catch; B, Effort; C, Raw CPUE; D, Cumulative catch.

\subsection{Temporal Structure of the Andes Fishery}

Fishing on the Andes Complex was not conducted uniformly throughout the year. There was a disproportionate amount of effort in the area between October-March (Tab. 3.1) and a distinct absence of effort from JuneAugust. This was a consequence of the fleet moving to target spawning orange roughy aggregations to maximise their catches. There is a prespawning orange roughy fishery to the northwest of the North-East hills 
(Fig. 3.1, (Anderson and Dunn, 2012)). The fleet then moves to the Spawning Box, where they target the spawning aggregations in early July to early August (Coburn and Doonan, 1994). A post-spawning fishery used to follow the fish leaving the spawning aggregation east towards seamount features, including the Andes Complex (Coburn and Doonan, 1994). In the spawning period, the entire fleet concentrates on fishing spawning roughy, leaving non-spawning areas like the Andes Complex unfished. In 1993 and 1994, the Spawning Box was closed to fishing. This restriction led to the first year-round fishery at the Andes Complex (Tab. 3.1). The changes in fishing pressure throughout the year and the years when the spawning fishery was closed, led to month and month $\mathrm{x}$ year interaction to be offered as predictors in my GLM. 
50 CHAPTER 3. EXPLORATORY ANALYSIS OF THE ANDES FISHERY

Table 3.1: The number of tows in each month at the Andes Complex from 1989 - 2013

\begin{tabular}{ccccccccccccc}
\hline \hline Year & Jan & Feb & Mar & Apr & May & Jun & Jul & Aug & Sep & Oct & Nov & Dec \\
\hline 1989 & 0 & 0 & 0 & 0 & 0 & 1 & 0 & 0 & 0 & 0 & 5 & 0 \\
1990 & 0 & 0 & 0 & 0 & 9 & 0 & 0 & 0 & 0 & 0 & 5 & 0 \\
1991 & 3 & 0 & 3 & 4 & 0 & 0 & 0 & 0 & 2 & 73 & 28 & 102 \\
1992 & 105 & 96 & 29 & 141 & 70 & 46 & 45 & 66 & 42 & 50 & 26 & 34 \\
1993 & 56 & 6 & 41 & 27 & 44 & 5 & 7 & 53 & 0 & 131 & 74 & 146 \\
1994 & 96 & 69 & 37 & 13 & 20 & 0 & 0 & 10 & 22 & 70 & 149 & 74 \\
1995 & 35 & 69 & 57 & 27 & 21 & 51 & 0 & 0 & 37 & 84 & 44 & 21 \\
1996 & 117 & 49 & 34 & 66 & 23 & 0 & 1 & 0 & 1 & 84 & 6 & 18 \\
1997 & 31 & 31 & 35 & 9 & 44 & 12 & 0 & 0 & 0 & 61 & 147 & 146 \\
1998 & 75 & 72 & 30 & 19 & 7 & 0 & 0 & 0 & 0 & 148 & 106 & 62 \\
1999 & 37 & 55 & 20 & 14 & 26 & 8 & 1 & 1 & 0 & 165 & 140 & 68 \\
2000 & 40 & 28 & 50 & 44 & 10 & 0 & 26 & 6 & 11 & 95 & 105 & 31 \\
2001 & 47 & 52 & 36 & 5 & 30 & 3 & 0 & 0 & 11 & 90 & 67 & 83 \\
2002 & 135 & 75 & 112 & 31 & 22 & 0 & 0 & 0 & 0 & 82 & 33 & 92 \\
2003 & 211 & 219 & 144 & 148 & 103 & 0 & 0 & 0 & 0 & 96 & 40 & 142 \\
2004 & 208 & 114 & 53 & 96 & 45 & 0 & 1 & 0 & 3 & 56 & 71 & 24 \\
2005 & 125 & 105 & 104 & 61 & 59 & 2 & 0 & 0 & 0 & 47 & 231 & 173 \\
2006 & 159 & 33 & 104 & 88 & 9 & 3 & 0 & 0 & 0 & 162 & 39 & 74 \\
2007 & 96 & 84 & 93 & 86 & 20 & 11 & 0 & 0 & 5 & 86 & 78 & 94 \\
2008 & 70 & 43 & 55 & 13 & 8 & 21 & 0 & 26 & 0 & 89 & 31 & 49 \\
2009 & 54 & 43 & 72 & 35 & 0 & 0 & 4 & 0 & 0 & 56 & 31 & 9 \\
2010 & 51 & 55 & 37 & 36 & 0 & 0 & 0 & 0 & 0 & 44 & 44 & 0 \\
2011 & 5 & 13 & 41 & 1 & 16 & 0 & 0 & 0 & 0 & 46 & 17 & 24 \\
2012 & 58 & 5 & 0 & 0 & 16 & 0 & 0 & 0 & 9 & 47 & 57 & 57 \\
2013 & 17 & 3 & 0 & 0 & 0 & 0 & 0 & 0 & 0 & 56 & 50 & 33 \\
\hline
\end{tabular}

\subsection{Vessels}

There have been 31 different vessels fishing for orange roughy over the 24 years the fishery has been active on the Andes Complex. However, the majority of the fishing has been conducted by a small number of these vessels. When the Andes Complex was first visited, there was a relatively high proportion of tows failing to capture orange roughy (Fig. 3.4). This can be attributed to skippers learning how to effectively fish the Andes Complex. Once the fishery was established, roughy were consistently cap- 
tured.
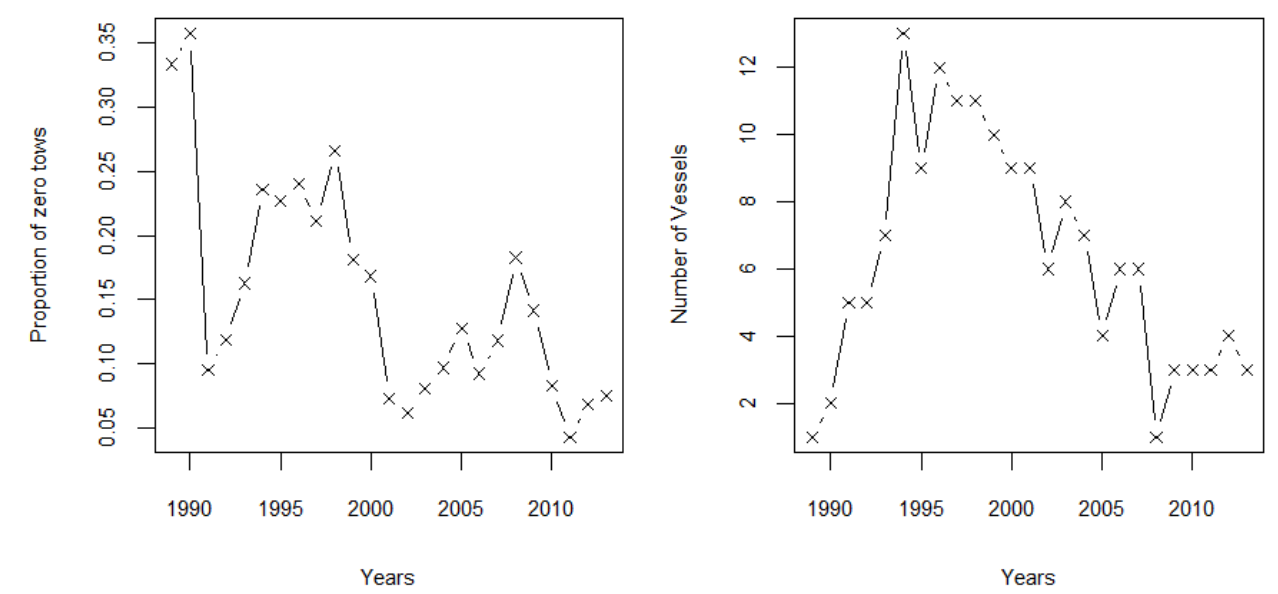

Figure 3.4: Left: The proportion of zero tows (not capturing roughy) from the fishery and right The number of vessels fishing in the Andes Complex between 1989 and 2013

The Andes Complex fleet is unlikely to fish consistently. Skippers will probably favour some locations and fish these sites at different times. This discrepancy needs to be removed from the CPUE index of abundance (Hilborn and Walters, 1992). Figure 3.5 illustrates the differences between individual vessel fishing patterns within the fleet in 1992. This only provides a snapshot in time, but it is evident that each vessel operates uniquely, targeting different features. The discrepancy between fishing patterns provides motivation to use vessel as a predictor to my generalised linear model for the Andes Complex. Prior to its addition to my model, a selection was undertaken to remove vessels which possessed insufficient data for the model to estimate an effect for. The criterion to provide a fair index of population abundance was vessels that had fished the Andes Complex 
52 CHAPTER 3. EXPLORATORY ANALYSIS OF THE ANDES FISHERY

for three years, with at least 20 tows per year. 


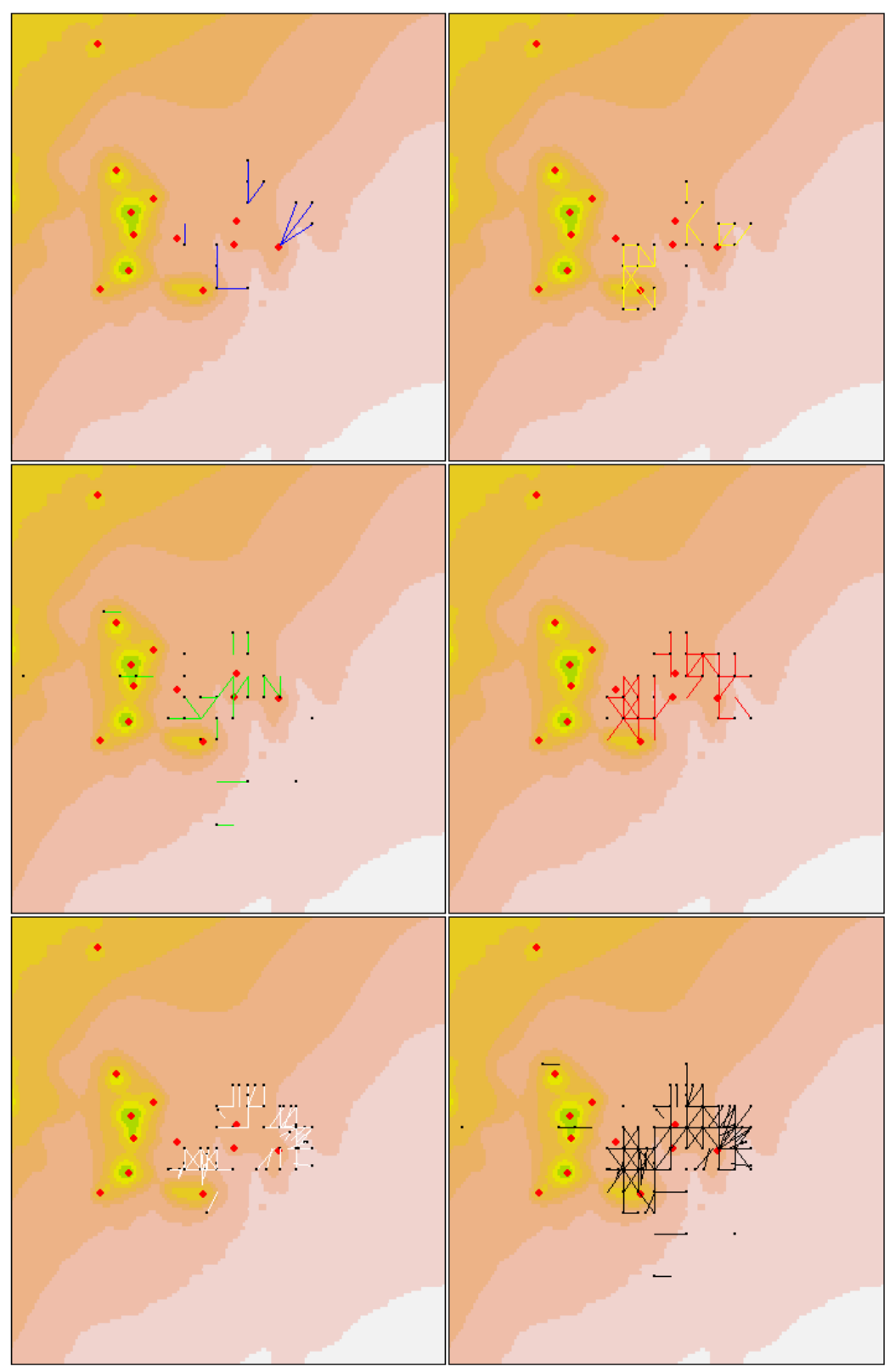

Figure 3.5: The fishing patterns of the six vessels targeting orange roughy at the Andes Complex in 1992. Each vessel has an individual plot with a unique colour for its towlines. Every tow conducted is plotted with black dots indicating the end position of each tow. 


\subsection{Subareas}

The fishery at the Andes Complex shows a relationship between CPUE and abundance that is characteristic of hyperdepletion (Dunn, 2011). The catalyst of this relationship is unclear, but there is evidence of fishers not homogeneously spreading their effort over the Andes and moving around areas within the complex. To investigate if this sequential depletion of features is driving a hyperdepleted index of abundance, the Andes complex was sub-divided. Initially, most of the fishing was conducted on either Possum or the four small seamounts on the eastern side of the Andes Complex (Fig. 3.2). Following this, the northern hills on the western Andes were fished and finally, the fleet started visiting the southern hills of the western side of the complex (Tab. 4.10).

Therefore, for analysis, the east and south Andes were separated due to the different topographical size of these features and the majority of the catch and effort was undertaken on the south Andes. The areas were subdivided by firstly making a distinction of the area that can be considered the geographical Andes Complex, which makes up the outer bounds of the subareas. Next, all tows were plotted out and lines were drawn to discern the areas. The lines were placed so tows would be grouped with the seamount that they were assumed to target (Fig. 3.6). There were tows in the overall dataset not within the geographical Andes region; an index of abundance from these tows was considered. These "other" tows originated from three distinct areas, the Aloha hill north of the Andes, a suspected canyon feature to the south of the Andes and the remainder from flat ground around the Andes. The other region tows were few in number 
and there was only one period from 2007 - 2009, where an index of abundance for this area was plausible (sufficient tows to form an abundance index (Tab. 3.3)), and therefore these tows were removed from the dataset. 


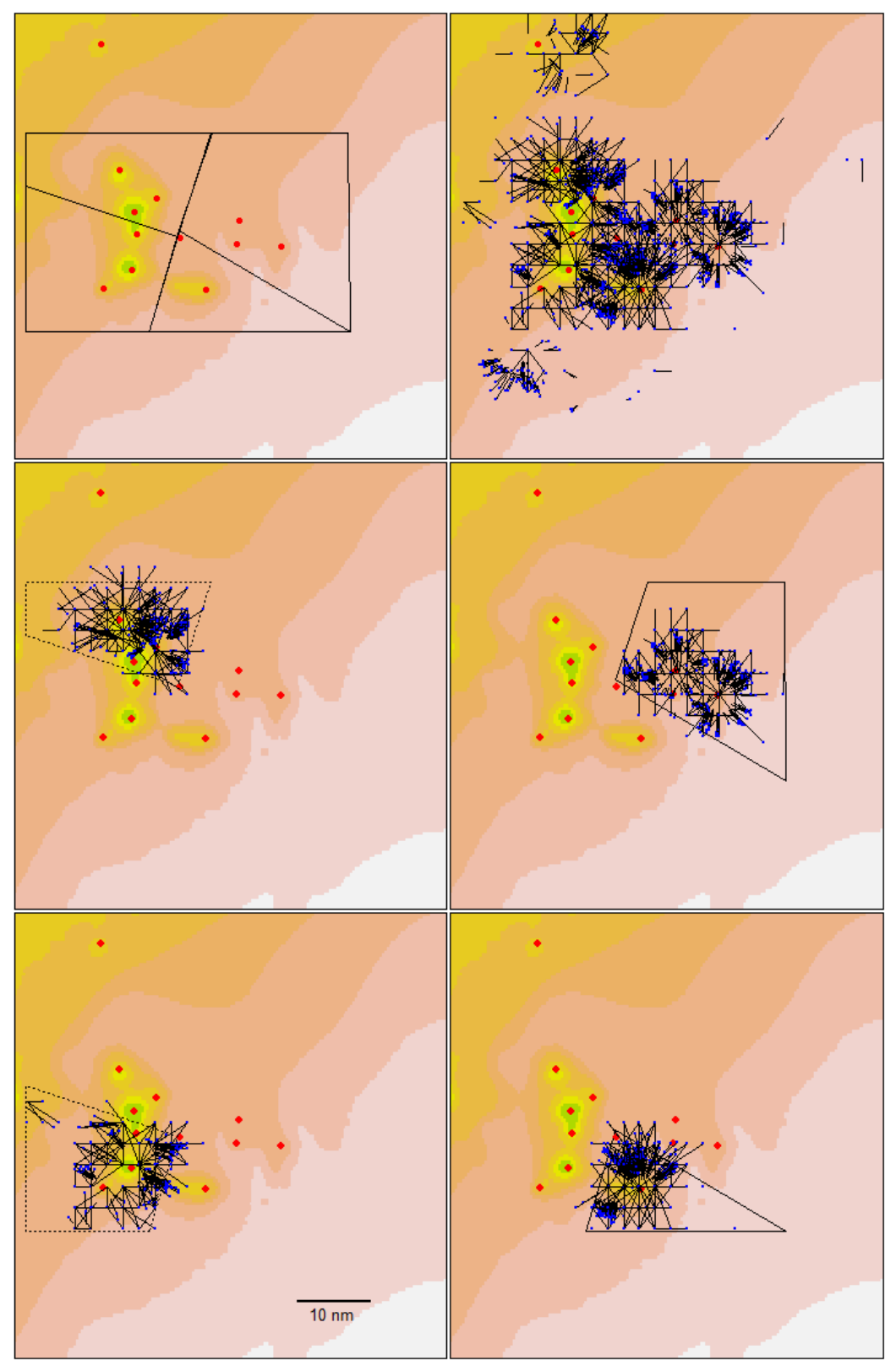

Figure 3.6: The geographical Andes Complex is identified and segregated into four subareas (top left). Each tow recorded in the wider Andes area from 1991 to 2013 has been plotted with start and end positions, with blue dots to show tow direction (top right). Each of the four subareas: northwest (middle left), east (middle right), southwest (bottom left) and south (bottom right), are plotted with all of their tows. A tow was allocated to an area based on its starting position. 
The temporal difference in fishing activity within each of the four Andes sub-areas is illustrated in Figure 3.7. A steep decline in raw CPUE is still evident in three subareas, indicating sequential fishing is not causing the apparent hyperdepletion relationship in CPUE. The south, east and northwest Andes subareas all showed sharp declines in CPUE in their initial years, whereas the southwest Andes fishery started in 1997 and generally followed the CPUE trends of the other subareas. The east, south and northwest subareas all sustained similar sharp declines in raw CPUE until 1995 and were remarkably similar by 1997 (fig. 3.7). Between 1995 and 2002, all the fisheries CPUE fluctuated around 3 tons per tow. From 2002 to 2009, all the regions displayed similar declines in CPUE and from 2009 to 2013, all the regions increased in CPUE. The cumulative catch illustrates that the South Andes region has been the source of the majority of the orange roughy removed from the Andes region. Since 2005, the annual catch across the regions has declined. This trend is evident in the flattening of the cumulative catch curves. The increase in CPUE from 2009 is not evident in the cumulative catch, indicating the increase in CPUE is associated with a reduction in effort. 

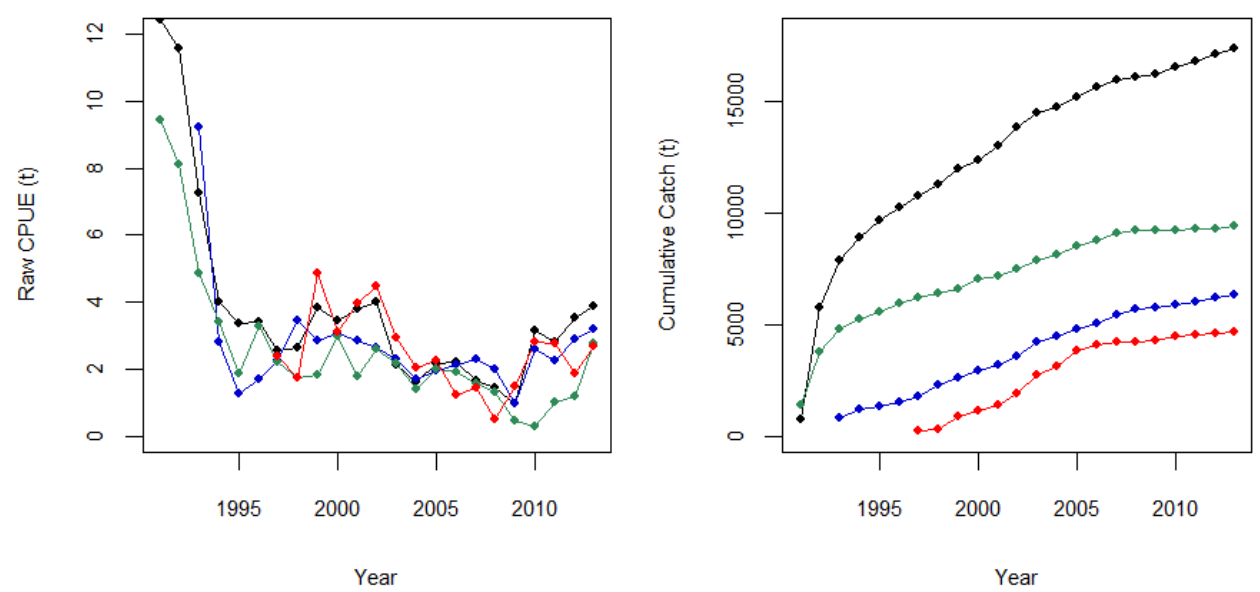

Figure 3.7: Left: Raw CPUE (tonnes/tow) of the four areas of the Andes and Right: the cumulative catch of these regions. The South Andes is plotted black, the East Andes green, the Northwest Andes blue and the Southwest Andes red.

The four subarea analysis is the area allocation which gave the best visual description for the observed changes in fishing pattern. However, alternate subarea permutations were considered and these models will be offered to the generalised linear model for selection. The alternate area splits considered are illustrated in Figure 3.8. A north-south split was included to account for the similarities evident in the raw CPUE trends between the south Andes and the southwest Andes. A west-east split considers that the west Andes is a single continuous feature, separating this feature from the rest of the Complex, whilst also taking into account the temporal differences between these locations. The final area split distinguishes between the size of the hills on the Andes Complex. The south, southwest and northwest hills have been amalgamated, as they are large hills (evident on the bathymetric map) and are distinct from the smaller 
topographical features of the east Andes.
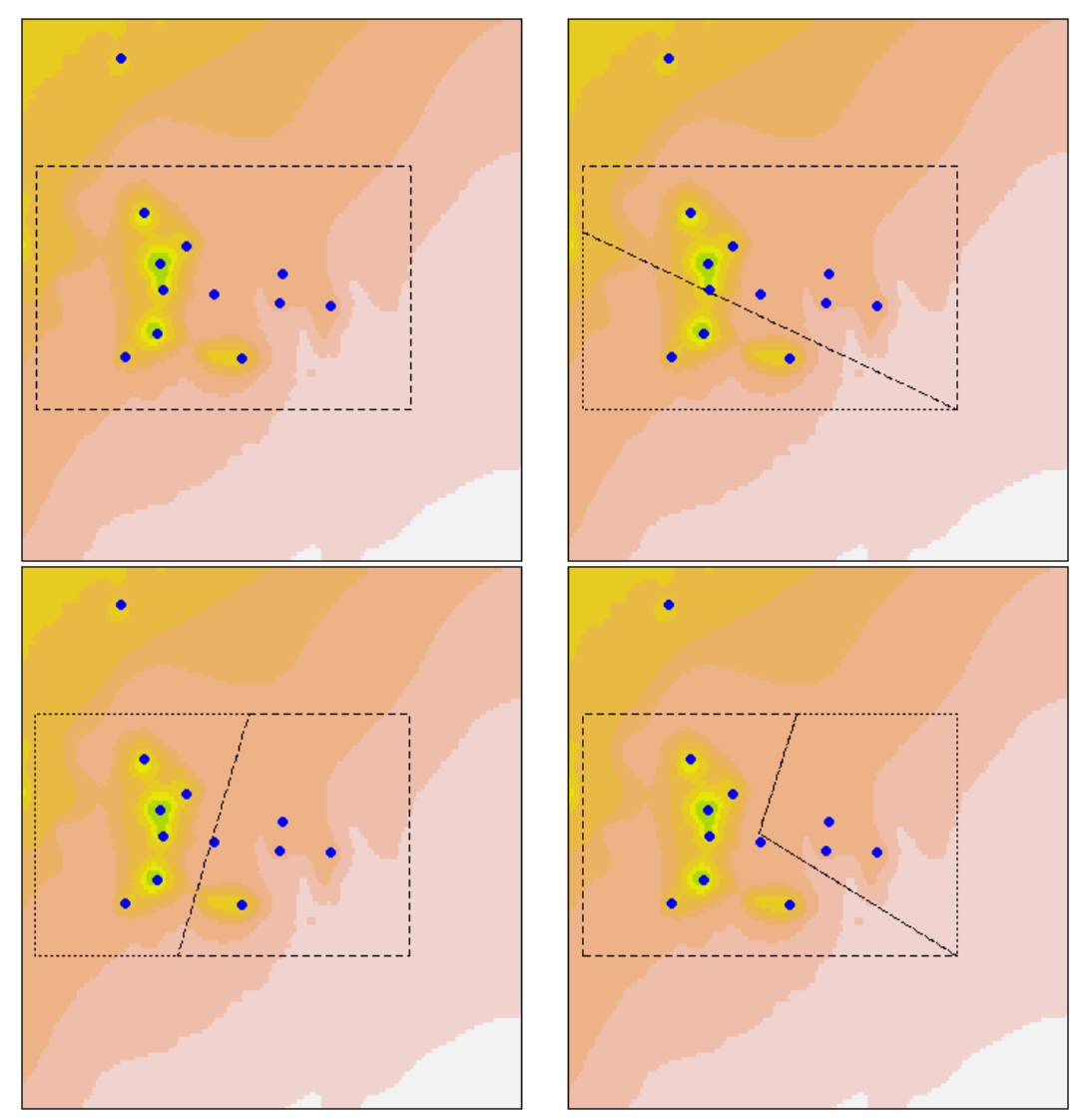

Figure 3.8: Four alternate area splits offered to the model for selection: no split (top left), north vs south (top right), east vs west (bottom left), and big seamounts vs small seamounts (bottom right).

\subsection{Generalised Linear Modeling}

I will use the delta method to create an overall abundance index or indices for the Andes Complex orange roughy fishery. The delta method consists of fitting two GLMs, a binomial GLM of the probability of captur- 
ing orange roughy at the Andes and a log-normal GLM of the catch, given that orange roughy were captured. The final delta abundance index is the product of the indices predicted by these models.

The models will be fitted with plausible explanatory variables, and then Akaike's Information Criterion (AIC) stepwise selection will be used to select the appropriate explanatory variables. For an explanatory variable to be accepted into a model, it must satisfy the following criteria: there must be a reduction in AIC or BIC, at least $1 \%$ additional deviance must be explained by the variable and it must contribute a plausible effect (Dunn, 2006). Bayes Information Criterion (BIC) will be utilised in scenarios where the aforementioned criteria do not clearly determine if an explanatory variable should be added or not. BIC should favour models with less explanatory variables, i.e. a parsimonious fit. Continuous explanatory variables were fitted as third order polynomials, to allow sufficient flexibility for their model fit. Categorical explanatory variables were fitted as factors and the response variable was catch per tow. Year was forced into both models, as a factor, then further predictors (Tab. 3.2) were added, based on the aforementioned criteria. The binomial and log-normal models for the Andes Complex were both offered the same potential predictors. 
Table 3.2: The potential predictors offered to the Generalised Linear Models

$\begin{array}{ll}\begin{array}{l}\text { Predictor } \\ \text { Year }\end{array} & \begin{array}{l}\text { Type } \\ \text { Categorical }\end{array} \\ \text { Month } & \text { Categorical } \\ \text { Day of year } & \text { 3rd order polynomial } \\ \text { Depth } & \text { 3rd order polynomial } \\ \text { Vessel ID } & \text { Categorical } \\ \text { Tow Duration } & \text { 3rd order polynomial } \\ \text { Subarea } & \text { Categorical } \\ \text { Subarea North/South } & \text { Categorical } \\ \text { Subarea East/West } & \text { Categorical } \\ \text { Subarea hill size } & \text { Categorical } \\ \text { Year * Month } & \text { Interaction } \\ \text { Year* Day of Year } & \text { Interaction } \\ \text { Year * Subarea } & \text { Interaction } \\ \text { Vessel ID * Subarea } & \text { Interaction }\end{array}$

$\begin{array}{ll}\text { Comment } & \text { df } \\ \text { Forced in } & 23 \\ & 12 \\ & 3 \\ \text { Vessels with } \geq 20 \text { tows for } \geq 3 \text { years } & 3 \\ & 12 \\ & 3 \\ \text { Distinguishes between North and South Andes } & 4 \\ \text { Distinguishes between East and West Andes } & 2 \\ \text { Distinguishes between Andes hills by size } & 2 \\ \text { Seasonal effect } & 276 \\ & 4380 \\ & 92 \\ & 48\end{array}$

\subsection{Andes Model}

The purpose of the exploratory analysis in this chapter was to get a thorough understanding of the Andes fishery sufficient to determine potential predictors for the GLM. Variables were offered for inclusion in the GLM if the explanatory analysis indicated a indicated a dependence of CPUE in the variable. Table 3.3 summarises the data that were used for the modeling process. 
Table 3.3: A summary of the data that goes into my GLM; the data is separated by subarea. For each subarea (EA east Andes, NWA northwest Andes, SA south Andes, SWA southwest Andes) the annual number of tows $(n)$, number of tows that capture ORH $(n(o r h))$ and median weight of catch $(\mathrm{kg})$ are displayed. Tows from outside the Andes (Other) are shown to illustrate the lack of data available to form an abundance index.

\begin{tabular}{|c|c|c|c|c|c|c|c|c|c|c|c|c|c|}
\hline & \multicolumn{3}{|c|}{ EA } & \multicolumn{3}{|c|}{ NWA } & \multicolumn{3}{|c|}{ SA } & \multicolumn{3}{|c|}{ SWA } & \multirow{2}{*}{$\begin{array}{c}\text { Other } \\
\mathrm{n}\end{array}$} \\
\hline Year & $\mathrm{n}$ & n(orh) & Med (kg) & $\mathrm{n}$ & $\mathrm{n}$ (orh) & Med (kg) & $\mathrm{n}$ & $\mathrm{n}$ (orh) & Med (kg) & $\mathrm{n}$ & $\mathrm{n}$ (orh) & Med (kg) & \\
\hline 1991 & 152 & 138 & 6750 & & & & 56 & 51 & 9000 & & & & 11 \\
\hline 1992 & 284 & 238 & 5000 & 21 & 18 & 6500 & 428 & 393 & 8000 & & & & 4 \\
\hline 1993 & 214 & 168 & 3000 & 76 & 67 & 4142 & 281 & 245 & 4000 & & & & 4 \\
\hline 1994 & 133 & 99 & 2000 & 119 & 84 & 2000 & 241 & 199 & 2000 & & & & 5 \\
\hline 1995 & 139 & 100 & 1000 & 70 & 46 & 1000 & 218 & 185 & 1000 & & & & 5 \\
\hline 1996 & 125 & 97 & 1216 & 94 & 56 & 580 & 155 & 132 & 1500 & & & & 5 \\
\hline 1997 & 112 & 79 & 1000 & 121 & 97 & 1000 & 204 & 162 & 1000 & 29 & 27 & 1800 & 38 \\
\hline 1998 & 106 & 68 & 1050 & 140 & 94 & 1793 & 183 & 146 & 2000 & 38 & 31 & 800 & 12 \\
\hline 1999 & 79 & 59 & 1000 & 106 & 85 & 1470 & 170 & 145 & 1500 & 120 & 108 & 2000 & 38 \\
\hline 2000 & 137 & 111 & 1000 & 88 & 70 & 2502 & 114 & 98 & 1208 & 78 & 71 & 1596 & 9 \\
\hline 2001 & 90 & 84 & 1000 & 97 & 91 & 1482 & 145 & 132 & 2211 & 58 & 57 & 2622 & 6 \\
\hline 2002 & 103 & 92 & 1000 & 130 & 120 & 1227 & 219 & 209 & 1558 & 120 & 119 & 2200 & 7 \\
\hline 2003 & 188 & 165 & 646 & 264 & 244 & 1000 & 286 & 270 & 1000 & 285 & 269 & 1036 & 56 \\
\hline 2004 & 151 & 128 & 500 & 144 & 133 & 740 & 166 & 149 & 600 & 182 & 173 & 1184 & 19 \\
\hline 2005 & 200 & 158 & 500 & 171 & 146 & 492 & 199 & 180 & 800 & 304 & 279 & 1064 & 32 \\
\hline 2006 & 146 & 126 & 509 & 113 & 97 & 1000 & 200 & 189 & 684 & 195 & 184 & 604 & 16 \\
\hline 2007 & 170 & 154 & 380 & 154 & 133 & 950 & 170 & 157 & 684 & 88 & 80 & 500 & 55 \\
\hline 2008 & 91 & 68 & 405 & 114 & 99 & 300 & 124 & 102 & 500 & 39 & 30 & 400 & 37 \\
\hline 2009 & 65 & 51 & 100 & 68 & 60 & 235 & 89 & 82 & 328 & 46 & 41 & 500 & 36 \\
\hline 2010 & 22 & 17 & 108 & 48 & 45 & 1400 & 68 & 59 & 1000 & 47 & 43 & 2000 & 9 \\
\hline 2011 & 7 & 5 & 200 & 21 & 21 & 1500 & 33 & 32 & 1250 & 16 & 16 & 1750 & 2 \\
\hline 2012 & 23 & 17 & 200 & 69 & 65 & 1500 & 78 & 75 & 1000 & 42 & 41 & 1000 & 3 \\
\hline 2013 & 39 & 34 & 500 & 31 & 30 & 2000 & 60 & 56 & 1400 & 25 & 24 & 1250 & 1 \\
\hline
\end{tabular}

\subsubsection{Binomial Model}

Table 3.4: Model selection for the Binomial GLM

$\begin{array}{ccccc}\text { Predictor } & \text { df } & \text { AIC } & \text { Deviance Explained } & \text { Additional Deviance Explained } \\ \text { Year } & 23 & 8601 & 4.1 \% & 3.8 \% \\ \text { Vessel ID } & 12 & 8356 & 7.2 \% & 3.1 \% \\ \text { Subarea } & 4 & 8293 & 8.1 \% & 0.8 \%\end{array}$




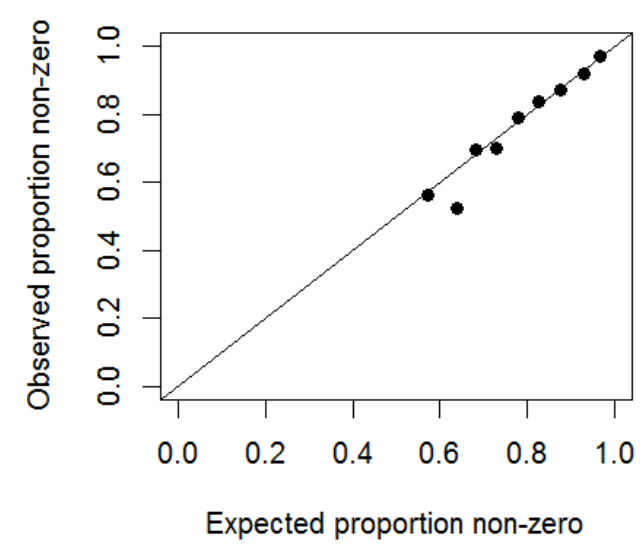

Figure 3.9: Observed and expected proportions of non-zero fits of the final Andes Binomial model.

The binomial GLM explained 7.7\% of the deviance in the data. Only year (forced in), vessel ID and subarea were selected into the model (Tab. 3.4). The small amount of deviance explained by the model suggests that instances of zero catch occur randomly, with few 0's in the data. Figure 3.9 shows an acceptable fit to the expected proportion on non-zero observations but is devoid of points below $p=0.5$, due to the lack of zero tows in the dataset. The binomial GLM prediction shows a slow decline in the probability of capturing roughy till 2000, then a relatively stable probability of capture at around $95 \%$ to date (Fig. 3.10). There was very little variation in this proportion between subareas, with all subareas displaying overlapping confidence intervals. The vessel effect shows one vessel with a substantially higher proportion of capture and another having a substantially lower proportion relative to the rest of the fleet. 
64 CHAPTER 3. EXPLORATORY ANALYSIS OF THE ANDES FISHERY
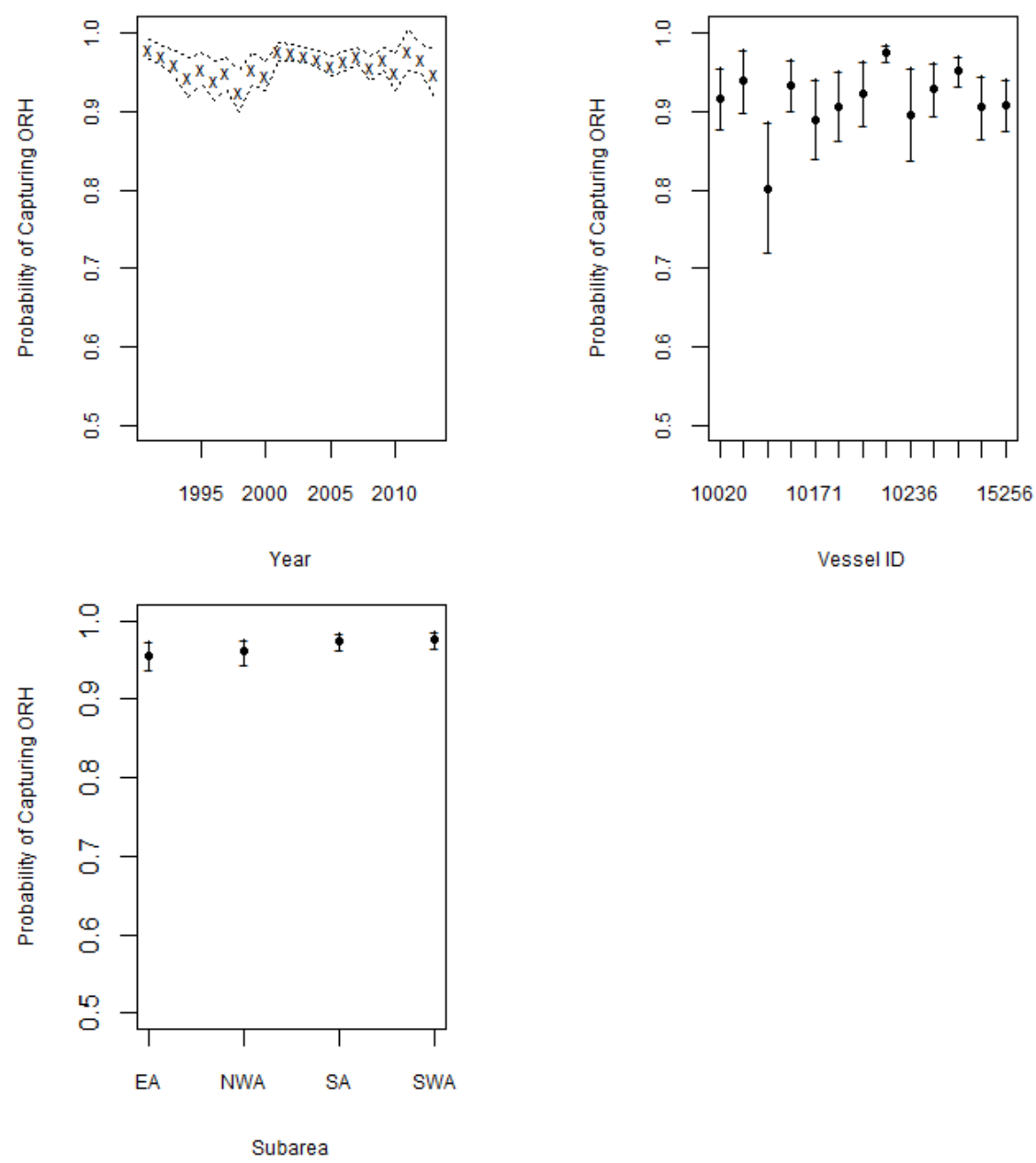

Figure 3.10: Binomial GLM predictions for the overall binomial index from 1991 to 2013 (top left), the vessel effect (top right) and the subarea effect (bottom left). Subareas are denoted: EA east Andes, NWA northwest Andes, SA south Andes and SWA southwest Andes. Each effect has been plotted with the other parameters set to their median value and with their corresponding $95 \%$ confidence intervals. 


\subsubsection{Normal Model}

Table 3.5: Model selection for Normal GLM

$\begin{array}{ccccc}\text { Predictor } & \text { df } & \text { AIC } & \text { Deviance Explained } & \text { Additional Deviance Explained } \\ \text { Year } & 23 & 36384 & 16.1 \% & 16.1 \% \\ \text { Vessel ID } & 12 & 36172 & 18.1 \% & 2.1 \% \\ \text { Subarea } & 4 & 36119 & 18.7 \% & 0.5 \% \\ \text { Year * Subarea } & 65 & 36116 & 20.5 \% & 1.6 \%\end{array}$

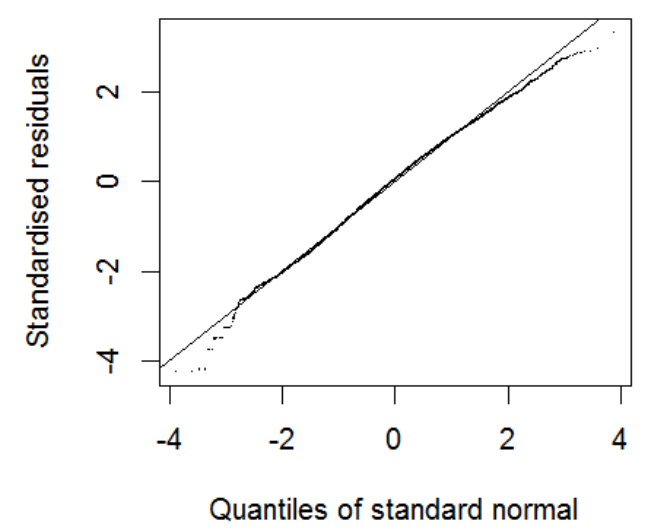

Figure 3.11: Normal quantile-quantile plot for the final Andes normal model fit.

The normal GLM explained $20.5 \%$ of the deviance with the predictor's year, vessel ID, subarea and year * subarea (Tab. 3.5). The q-q plot shows a reasonable fit of the residuals to the fitted line, only straying from normality outside two standard deviations (Fig. 3.11). The GLM predictions for the four Andes subareas show similar trends in abundance; initially a sharp decline lasting $4-5$ years, and stopping in the mid 1990s. The index then shows a very gradual decline for around 10 years. From 2005 to date, the index has stabilised and all the regions display a slight increase 
(Fig. 3.12). The difference in trend between the four Andes subareas is almost indecipherable; ignoring the difference in the start point of each area, they are significant yet very similar. The south Andes and northwest Andes abundance indicies both peak in their second year, which implies some skipper fishery learning of the area was required to attain a maximum index. The four subareas have similar overall effects, with their confidence intervals overlapping, although the east Andes is lower than the others. The individual vessel effects are broadly similar; three vessels attain a higher index than average and one vessel has a lower index. Overall, the vessel effects show little contrast, as the majority of confidence intervals overlap (Fig. 3.12). 

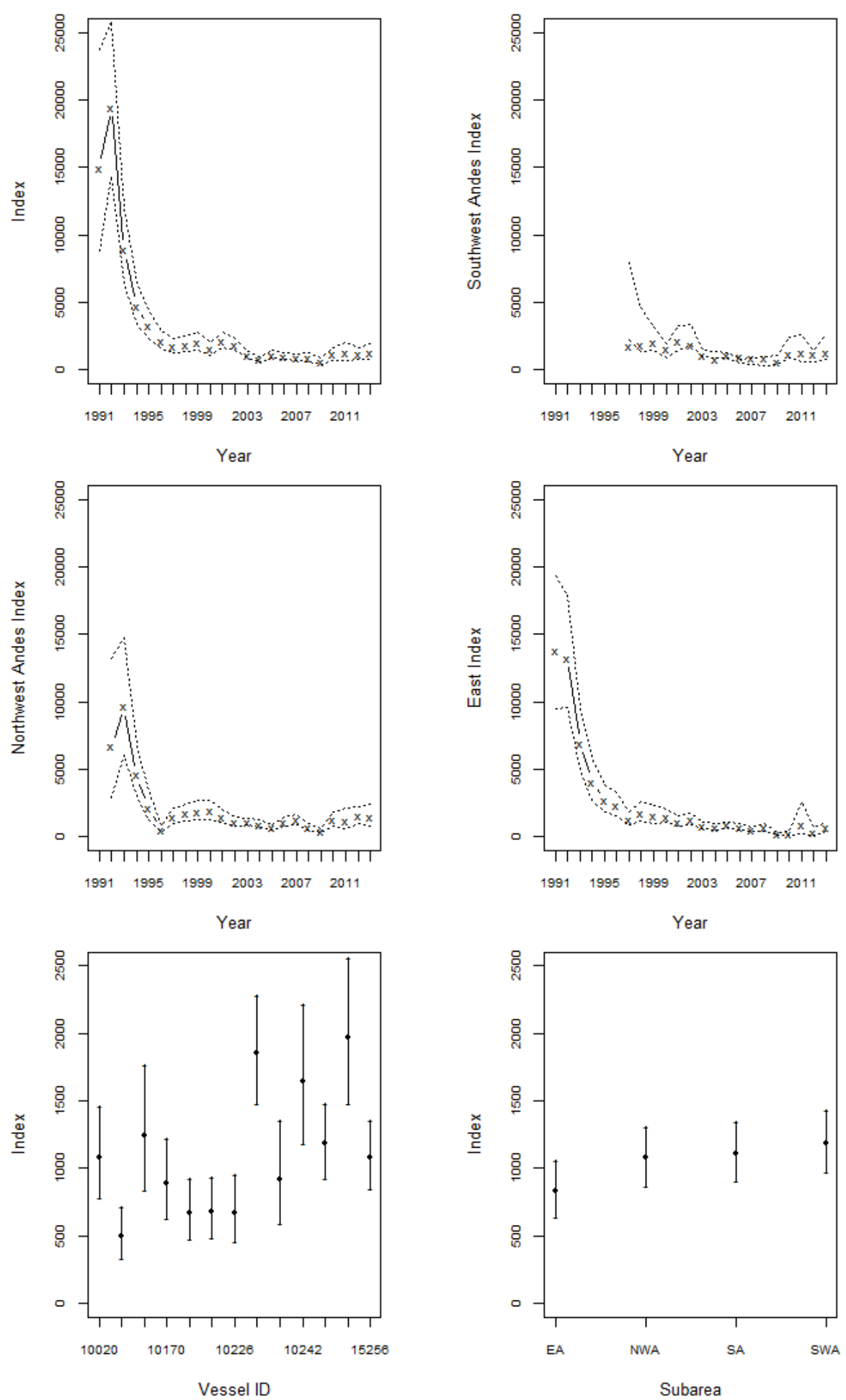

Figure 3.12: Normal Generalised Linear Model predictions; south Andes (top left), southwest Andes (top right), northwest Andes (middle left), east Andes (middle right) from 1991-2013; vessel effects (bottom left) and subarea effects (bottom right). Subareas are denoted: EA east Andes, NWA northwest Andes, SA south Andes and SWA southwest Andes. Each effect has been plotted with the other parameters set to their median value and with its corresponding $95 \%$ confidence intervals. 


\subsubsection{Combined Model}

The predictions from the combined model index show an initial large decline in the abundance index of the Andes orange roughy fishery, with the abundance index declining six-fold in the first five years (Fig. 3.16). The speed of this decline reduces after 1994, but a gradual decline continues until 2010 (Fig. 3.16). After 2010, there is a small uplift in the CPUE index, although this trend in the log-normal was reduced by the addition of the binomial index. The predicted values of Vessel ID show that the fleet has similar catches, with three vessels displaying a higher abundance index and one vessel a lower abundance index. The subarea index showed no difference between the northwest and east Andes, while the southwest and south Andes had a higher abundance indicies, the southwest was the highest. 

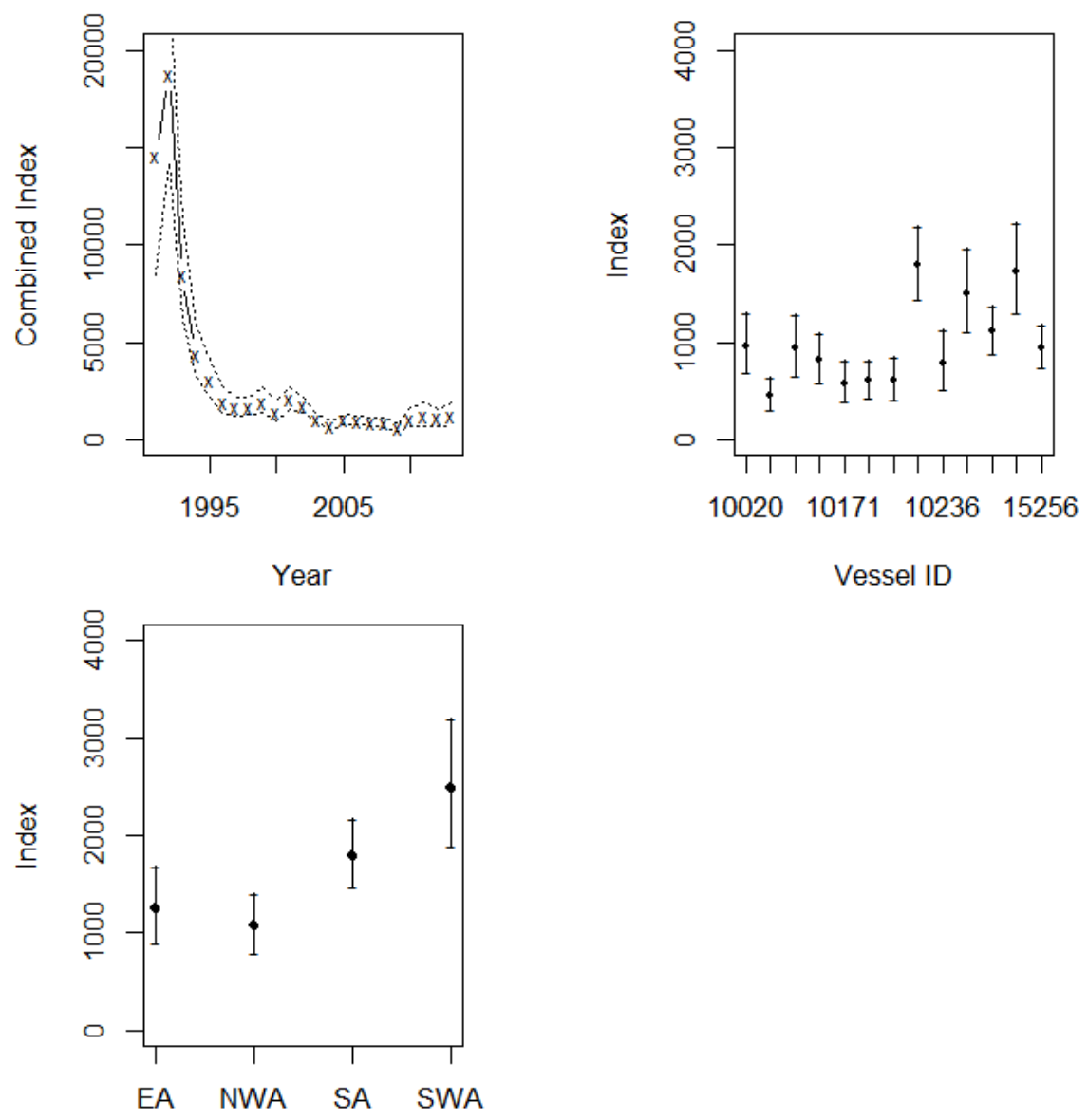

Subarea

Figure 3.13: Final abundance indicies for the combined model; year from 1991 to 2013 (top left), vessel (top right), and subarea (bottom). Each effect is plotted with the other parameters set at their median values and with its corresponding bootstrapped coefficients of variation.

The weighted mean of the raw unstandardised CPUE and untransformed GLM predictions are compared in figure 3.14. They show the effect of the standardisation process on the abundance index. Standardisation did not have a large effect on the northwest, east and south subareas. 
Generally its effect smoothed some of the sharp changes in abundance index. However, in the southwest Andes subarea there is a relatively large difference between the trajectories of the two indicies, with the raw CPUE under-predicting the standardised index for the first three years of the fishery, and then over-predicting the standardised index from 2002 to 2009.
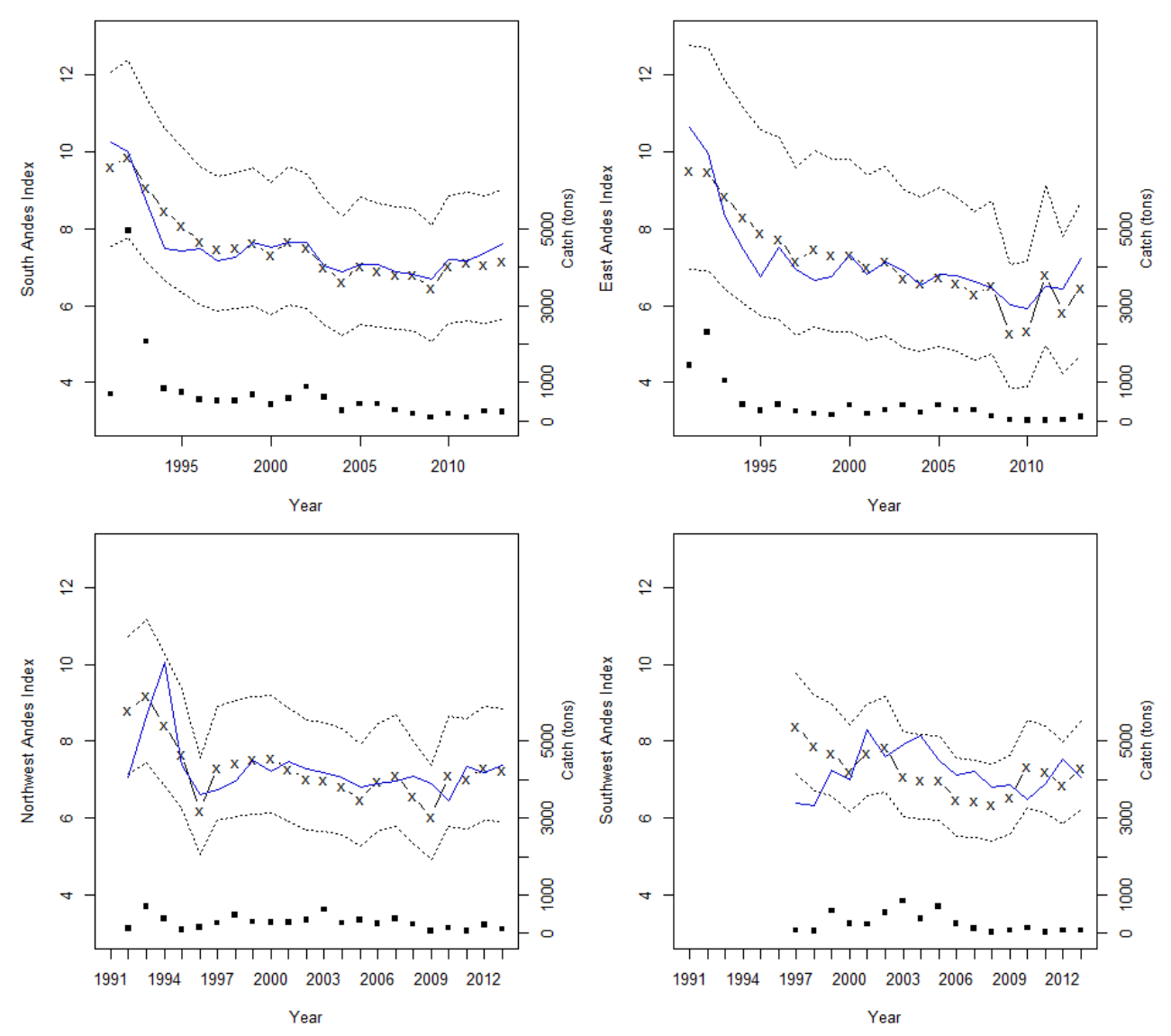

Figure 3.14: Comparison between raw unstandardised CPUE (blue) and the untransformed normal GLM predictions (x) with their corresponding 95\% confidence intervals (dotted lines) for the Andes four subareas; south (top left), east (top right), northwest (bottom left), and south west (bottom right), from 1991 to 2013. The third axis displays the catch of orange roughy (tons), which are plotted at squares as the bottom of the graph. 
Subarea explained only $0.8 \%$ additional deviance in the the binomial model (Tab. 3.4) and $0.5 \%$ in the normal model. The interaction was selected and explained $2.1 \%$ additional deviance but it has an additional 109 degrees of freedom (Tab. 3.5). The large number of degrees of freedom in the subarea $x$ year interaction indicate that if a different model selection criteria was utilised, it would be unlikely for this interaction to be selected. This is due to the interaction being penalised for its large number of degrees of freedom, that do not outweigh the deviance it explains. BIC applies a harsher penalty to the number of parameters in the model. Under BIC selection the interaction effect would not be included in the final model (Tab. 3.6). The year x subarea interaction visually display similar trends in the abundance index, apart from the different starting years in the northwest and southwest Andes (Fig. 3.12). The four subareas are plotted atop of each other in figure 3.15; there are no substantial differences in index trend between the four regions. The predicted individual effect of subarea is visually minor the binomial and normal models (Figs. $3.12,3.10)$ coupled with this subarea has a marginal significance in both models. Based on the marginal significance, $B I C$ rejection of the subarea $x$ year interaction, and the small visual difference in the abundance trends and the very large degrees of freedom when estimating the coefficients, subarea was removed from the final model. This favours a parsimonious final CPUE index. 
Table 3.6: Reanalysis of selection for Normal model

$\begin{array}{cccccc}\text { Predictor } & \text { df } & \text { AIC } & \text { Deviance Explained } & \text { Additional Deviance Explained } & \text { BIC } \\ \text { Year } & 23 & 36384 & 16.1 \% & 16.1 \% & 8150 \\ \text { Vessel ID } & 12 & 36172 & 18.1 \% & 2.1 \% & 7910 \\ \text { Subarea } & 4 & 36119 & 18.7 \% & 0.6 \% & 7845 \\ \text { Year * Subarea } & 65 & 36116 & 20.5 \% & 1.6 \% & 7871\end{array}$

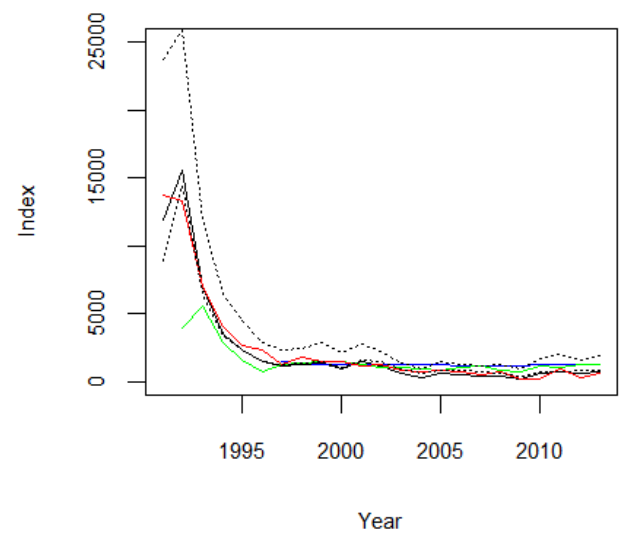

Figure 3.15: Comparison of the differences in abundance indices between the four Andes subareas from 1991 to 2013: South Andes (black), East Andes (red), Northwest Andes (green) and Southwest Andes (blue). The subareas have been standardised, then scaled to the geometric mean of the East Andes; the CV for the East Andes has been added to the plot.

\subsubsection{Final Model}

Table 3.7: The final Binomial Model

$\begin{array}{ccccc}\text { Predictor } & \text { df } & \text { AIC } & \text { Deviance Explained } & \text { Additional Deviance Explained } \\ \text { Year } & 23 & 8601 & 3.8 \% & 3.8 \% \\ \text { Vessel ID } & 12 & 8356 & 6.9 \% & 3 \%\end{array}$


Table 3.8: The final Normal Model

$\begin{array}{ccccc}\text { Predictor } & \text { df } & \text { AIC } & \text { Deviance Explained } & \text { Additional Deviance Explained } \\ \text { Year } & 23 & 36384 & 16.1 \% & 16.1 \% \\ \text { Vessel ID } & 12 & 36172 & 18.1 \% & 2.1 \%\end{array}$

The final binomial and normal models for orange roughy abundance at the Andes Complex show a slight reduction in deviance explained by removing the subarea effect and subarea $x$ year interaction (Tabs. 3.7, 3.8). In both models, fishing year explains the majority of the deviance, and the remainder is made by vessel. The binomial index shows little change in the probability of capture for orange roughy throughout the time series (Fig. 3.16). The normal index of abundance shows a high initial two years, with a peak in 1992; these two years have substantially larger confidence intervals than the rest of the time series (Fig. 3.16). After 1992, the index shows a steep decline until 1996. From 1996_2002, the abundance index remains stable, before another gradual decline until 2009. In 2009, there was a slight increase in the abundance index to date. The combination of the normal and binomial models is displayed in the combined index. The binomial index is stable, so the combined index reflects the normal index. The effect of vessel is minor, explaining little deviance and with most vessels having overlapping confidence intervals, although three vessels appear to be more successful than the majority (Fig. 3.16). 

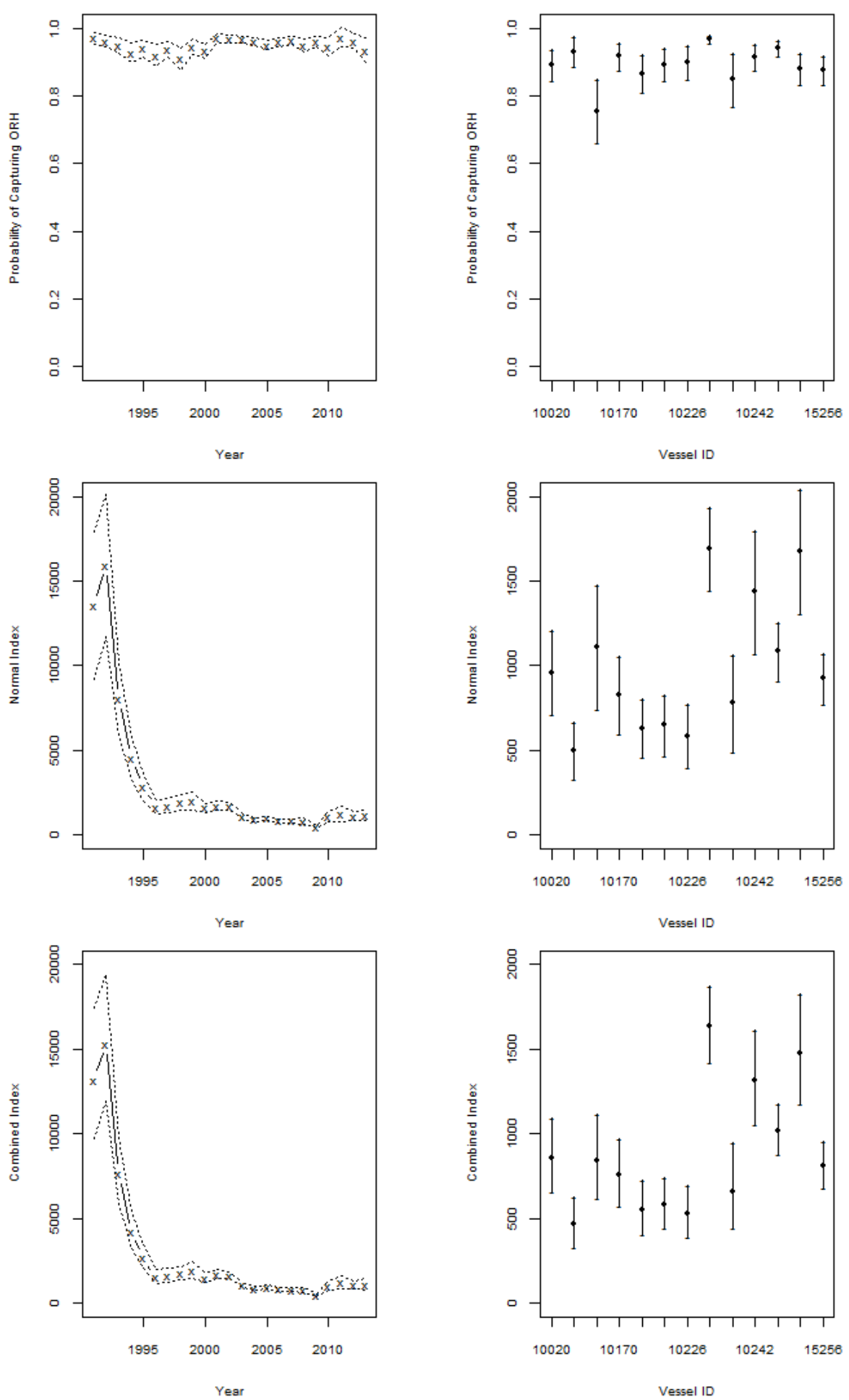

Figure 3.16: Final model output for the Andes Complex, binomial model, first row, normal model, second row and combined model bottom row. The model predictions are plotted with their corresponding CV's; these are bootstrapped for the combined model. Predictions are based on using the Vessel ID 10227 for Year and the Year 2002 for Vessel ID 
Table 3.9: Final GLM table; binomial, normal and combined models displayed with their Coefficients of Variation (CVs)

$\begin{array}{ccccccc}\text { Year } & \text { Binomial } & \text { CV } & \text { Normal } & \text { CV } & \text { Combined } & \text { CV } \\ 1991 & 0.858 & 6 \% & 6256.3 & 24 \% & 6099.4 & 24 \% \\ 1992 & 0.823 & 6 \% & 7344.2 & 22 \% & 7001.5 & 23 \% \\ 1993 & 0.772 & 7 \% & 3717.6 & 22 \% & 3418.8 & 23 \% \\ 1994 & 0.703 & 10 \% & 2082.0 & 22 \% & 1812.9 & 25 \% \\ 1995 & 0.747 & 8 \% & 1307.2 & 22 \% & 1179.5 & 24 \% \\ 1996 & 0.681 & 10 \% & 753.9 & 21 \% & 643.7 & 25 \% \\ 1997 & 0.735 & 8 \% & 797.0 & 21 \% & 712.1 & 24 \% \\ 1998 & 0.648 & 10 \% & 889.0 & 20 \% & 736.8 & 23 \% \\ 1999 & 0.764 & 8 \% & 936.8 & 22 \% & 856.2 & 24 \% \\ 2000 & 0.720 & 7 \% & 735.9 & 19 \% & 649.5 & 20 \% \\ 2001 & 0.859 & 4 \% & 796.5 & 18 \% & 777.3 & 19 \% \\ 2002 & 0.849 & 5 \% & 779.7 & 19 \% & 755.8 & 20 \% \\ 2003 & 0.842 & 4 \% & 503.8 & 18 \% & 486.2 & 20 \% \\ 2004 & 0.818 & 5 \% & 417.0 & 17 \% & 396.0 & 19 \% \\ 2005 & 0.778 & 6 \% & 453.4 & 18 \% & 418.8 & 20 \% \\ 2006 & 0.811 & 6 \% & 390.8 & 19 \% & 369.5 & 20 \% \\ 2007 & 0.831 & 5 \% & 383.8 & 19 \% & 367.7 & 20 \% \\ 2008 & 0.774 & 7 \% & 372.1 & 20 \% & 342.7 & 22 \% \\ 2009 & 0.823 & 6 \% & 219.6 & 20 \% & 209.2 & 22 \% \\ 2010 & 0.757 & 9 \% & 502.8 & 21 \% & 457.1 & 24 \% \\ 2011 & 0.873 & 8 \% & 567.7 & 25 \% & 558.9 & 24 \% \\ 2012 & 0.820 & 7 \% & 512.6 & 21 \% & 487.6 & 23 \% \\ 2013 & 0.727 & 11 \% & 535.1 & 22 \% & 475.1 & 25 \%\end{array}$

The final binomial index shows low coefficients of variation around its estimates and the normal abundance index has coefficients of variation around 20\% (Tab. 4.10). The combined index of abundance has coefficients of variation of $\tilde{2} 0 \%$. 
76 CHAPTER 3. EXPLORATORY ANALYSIS OF THE ANDES FISHERY 


\section{Chapter 4}

\section{Model Fitting and Comparison}

\subsection{Model Assessment}

Once each SPM was run through the maximum likelihood estimation process, the fit needed to be assessed. There is no single measure of fit that summarizes all aspects of model fit (Hilborn and Mangel, 1997), therefore it is important to have a range of criteria to determine the best canditate model or models.

\subsubsection{Information Criteria}

The principle of Occam's razor states that among competing hypotheses that predict equally well, the one with the fewest assumptions should be selected. Here I had a salient application. My desire to attain a better model fit led to the addition of parameters, but I could enter a scenario where my SPM might be overfitted. Overfitting is the use of models that violate parsimony (Hawkins, 2004); that is they add unnecessary complex- 
ity. If I keep adding parameters to my model, the deviance explained will increase but the additional parameters may not be useful or cannot be estimated with any precision. Information criteria use the value of the likelihood and the number of parameters in the model to determine a value for the candidate model: these values are then compared between models with the lowest value desirable. Here I use three different information criteria, with different penalties to assess my candidate models. Akaike's Information Criterion $(A I C)$ is the simplest; it penalises complexity by adding two times the number of parameters to the likelihood (Eq. 4.1). $A I C$ may perform poorly if there are too many estimated parameters relative to the sample size. A solution to this is to use small sample $A I C$ or $A I C_{c}$ (Burnham and Anderson, 2002). $A I C_{c}$ has a bias correction for the relationship between $k$, the number of parameters in the model and $n$, the amount of data in the model (Eq. 4.2). Bayes Information Criterion (BIC) was used as an alternate to $A I C$ and $A I C_{c}$ because it applies a harsher penalty to the number of parameters in a candidate model, which may be important in avoiding overfitting (Eq. 4.3). The three criteria are given by

$$
\begin{aligned}
& A I C=-2 \log (L)+2 k \\
& A I C_{c}=-2 \log (L)+2 k+\frac{2 k(k+1)}{n-k-1} \\
& B I C=-2 \log (L)+k \log (n)
\end{aligned}
$$

where $L$ is the likelihood of a candidate model, $k$ the number of parameters in the model and $n$ the number of data points in the model. 


\subsubsection{Confidence Intervals and Likelihood Profiles}

Confidence intervals for the SPM model parameters $(r, K, q, \sigma)$ and the estimated abundance index $\left(\hat{I}_{t}\right)$, were calculated using bootstrapping (Haddon, 2010). Confidence intervals were initially formed based on the square root of the diagonal elements of the inverse hessian matrix. These intervals were found to be excessively tight (e.g. for $K=20062$ an interval of (20061, 20063)). The hessian matrix intervals were thought to be problematic because the interval assumed a normal distribution, whereas the data were not normally distributed. The bootstrapping procedure re-sampled (with replacement) the residuals from the estimated index (Eq. 4.4), and these residuals were then applied to the estimated abundance index to form a new "observed" index, $\hat{I}_{t}^{*}$ (Eq. 4.5).

$$
\begin{gathered}
e_{t}=\log \left(\frac{I_{t}}{\hat{I}_{t}}\right) \\
\hat{I}_{t}^{*}=e_{t} \times \hat{I}_{t}
\end{gathered}
$$

where $e_{t}$ is the residual for time $t, I_{t}$ is the observed abundance in time

$t, \hat{I}_{t}$ is the estimated abundance index in time $t$ and $\hat{I}_{t}^{*}$ is the new bootstrapped observed index at time $t$.

The new observed index $\left(\hat{I}_{t}^{*}\right)$, replaced the observed index in the negative log-likelihood function; optim then carried out the MLE and the new parameter estimates $\left(r^{*}, K^{*}, q^{*}, \sigma^{*}\right)$ were stored. Confidence intervals were created by repeating the bootstrapping process 1000 times, the 95\% confidence interval are the 25 th and 975 th estimates from the sorted bootstrapped parameter estimates. The bootstrapped parameter estimates 
$\left(r^{*}, K^{*}, q^{*}, \sigma^{*}\right)$ were entered into the SPM equation for the hypothesis in question which produced intervals around the estimated abundance in$\operatorname{dex}\left(\hat{I}_{t}\right)$. The minimum log-likelihood parameter estimates were not positioned in the centre of the bootstrapped confidence intervals because asymmetric nature of their distribution. They sat closer to the lower confidence interval, as a consequence of the log-normal distribution having a skew toward higher values.

Likelihood profiles also provide a means of reconstructing a more robust confidence interval when sample sizes were small or parameters of interest did not follow a normal distribution. A profile was created by fixing the parameter at a specified value, then optimising the likelihood with respect to all other parameters. Here I profile the biological parameters $r$ and $K$ over their respective plausible ranges primarily to provide a visual confirmation that my model has converged at a minima and after some initial problems with convergence in the base model. A minimum shown on a likelihood profile may not be equal to that found through global minimisation, since a profile is a single transect that does not represent the overall multi-dimensional parameter space. Profiling variables was problematic when their plausible range was unknown. This resulted in convergence issues attributed to extreme parameter values and poor choice of starting values. Problems were also encountered with parameters which possessed likelihood surfaces with minima adjacent to large likelihood increases ("walls"). In this situation, a penalty function was added to the likelihood to ensure a minimum was reached prior to the likelihood wall (Eq. 4.6). 


$$
P_{t}=0.1 \times \frac{C_{t}}{\left(B_{t}-C_{t}\right)}
$$

where $P_{t}$ is the penalty in time $t, C_{t}$ is the catch in time $t$ and $B_{t}$ is the biomass in time $t$.

A wall in the likelihood profile is caused by the SPM estimating biomass as low as possible to fit the abundance index. If the biomass gets too low, the entire biomass can be removed by catches in subsequent years. As a consequence, the biomass becomes negative and the likelihood jumps, creating a wall. The penalty function penalises the negative log-likelihood value as biomass gets reduced relative to catch. The penalty function ensured profiles reached a minima but it also will create a bias in the loglikelihood transects and move the position of minima away from walls. With some profiles, the optimisation performed so poorly that manual profiling was required to ensure a minima was achieved. Manual profiling was conducted by the same means, but for each value in the profile, starting values were randomly varied 15 times and the minimum likelihood achieved from these runs was the value profiled.

\subsubsection{Visual Assessment}

The estimated index of abundance from the observation model is equivalent to the observed index of abundance from my Andes Complex orange roughy catch-per-unit-effort analysis (Chapter 3)). Candidate models were assessed visually by comparing the the predicted abundance index and the observed abundance index. Standardised residuals $\left(e_{t}=\right.$ 
$\left.\log \left(I_{t} / \widehat{I}_{t}\right)\right)$ were plotted alongside the abundance indicies to show any consistent patterns in under- or over-estimation. The plots of standardised residuals had horizontal lines at 2 standard deviations of the residuals; 2 standard deviations captures $95 \%$ of the expected distribution of the residuals. Confidence intervals were plotted around the expected abundance indicies. More precise model estimates are indicated by tighter confidence intervals.

\subsection{Hypothesised Models}

In section 4.2 I present the results of fitting models corresponding to my hypotheses. Comparison of these models is made in section 4.3.

\subsubsection{Closed Population Model}

The first model to test is the null model, a closed population of orange roughy at the Andes Complex. This model is a standard Schaefer model and was the simplest model to be examined.

The parameter estimates for the closed population model are displayed in table 4.1 with their $95 \%$ confidence intervals. A penalty function was applied to the Closed Population Model because of observed walls in the negative log-likelihood profiles and to assist convergence (Fig. 4.1). 
Table 4.1: Parameter estimates and their corresponding 95\% confidence intervals for Closed Population Model, estimates with the subscript $p$ have a penalty added to their likelihood. (* parameter has hit a bound)

\begin{tabular}{llll} 
Parameter & Estimate & Lower & Upper \\
\hline$K$ & 15569 & 14138 & 27674 \\
$r$ & 0.4252 & 0.1211 & 0.5228 \\
$q$ & 0.2043 & 0.0748 & 0.2601 \\
$\sigma$ & 0.4379 & 0.2709 & 0.5144 \\
\hline$K_{p}$ & 20045 & 15828 & 49887 \\
$r_{p}$ & 0.2478 & $0.0000^{*}$ & 0.3996 \\
$q_{p}$ & 0.1238 & 0.0308 & 0.2044 \\
$\sigma_{p}$ & 0.4262 & 0.2564 & 0.5530
\end{tabular}

Likelihood profiles for parameters $r$ and $K$ were estimated (Fig. 4.1); both profiles showed log-likelihood walls adjacent to their minimum loglikelihood values. The position of the log-likelihood walls made convergence difficult. Their position indicated a biomass limitation problem that was addressed by the addition of penalty function to the log-likelihood (Eq. 4.6; Fig. 4.1). The penalty function improved the estimation of the profiles for both $r$ and $K$, although it added a small bias to the estimates. The position of the minimum for $K$ was biased slightly higher, whereas the position of the minimum for $r$ was biased lower (Fig. 4.1). The estimates for all parameters had wider confidence intervals when the penalty function was applied. 

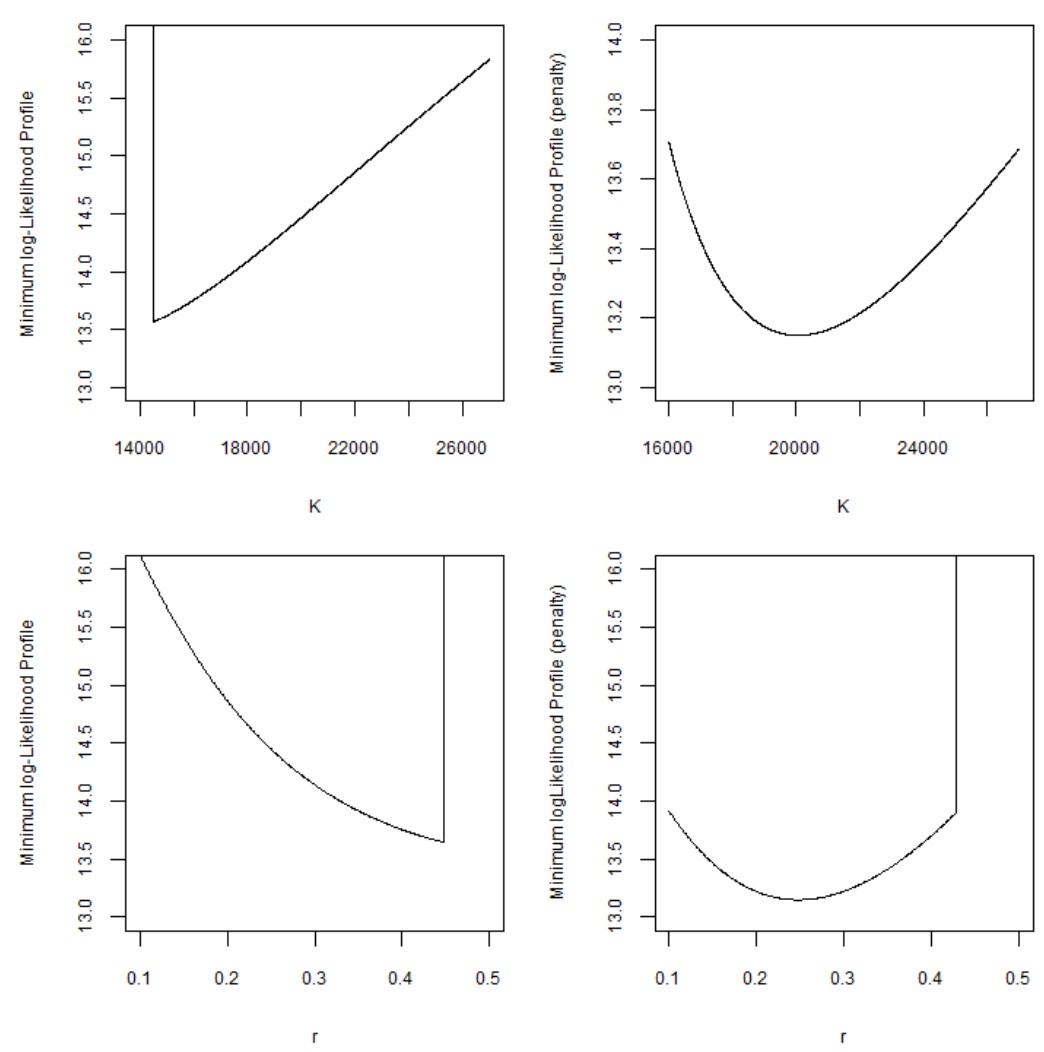

Figure 4.1: Minimum log-likelihood profiles of $\mathrm{r}$ from $0-0.5$ (bottom); and K from 1400026000 tons. The left hand panels are standard profiles whereas the right hand panels have a penalty added to the likelihood function.

The Closed Population Model displayed a poor fit to the CPUE index, especially from 1991 to 1995 , where it underestimated the decline in the index (Fig. 4.2). The difference was clear in the residual plot, where there was a constant underestimation of biomass in the early years of the fishery. From 1995 to 2010, the model overestimates the index. Influential points were evident in 1992 and 1993 with standardised residuals outside 2 standard deviations (Fig. 4.2). There was a more satisfactory fit from 1995 onwards but the poor fit in the early years is a classic feature of hy- 
perdepletion (Dunn and Devine, 2010).
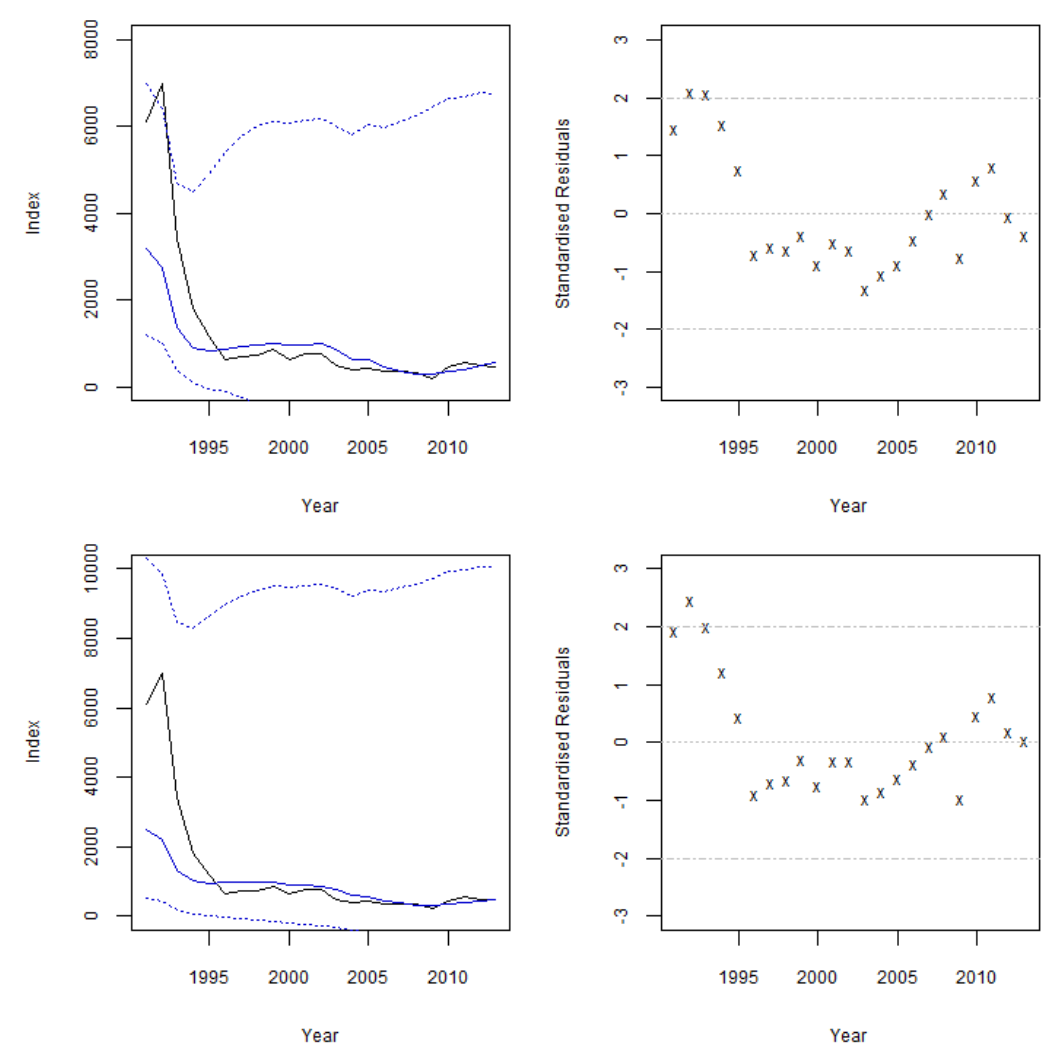

Figure 4.2: Estimated Andes orange roughy population index (blue) with corresponding $95 \%$ confidence intervals for the Closed Population Model and the observed population index (black) from 1991 - 2013 (left). The residual plot shows the difference between the estimated and observed index; the central dashed line indicates no difference (zero) and the periphery dashed lines indicate $+/-2$ standard deviations of the residuals. The top panels show the unmodified abundance index, whereas the bottom panels have a penalty added to the likelihood function.

The Andes Complex fishery had very high initial catches that dropped drastically. This was followed by relatively stable lower catch levels to date (Fig. 4.2). For this pattern to occur, the biomass initially needs to decline rapidly due to removals from catch. This indicated a small biomass 
for the Andes Complex but the continued flat biomass trend to date required a higher biomass; the two trajectories therefore conflicted. To investigate this suspected characteristic, a retrospective analysis was performed, where data were removed from the end of the time series, and the model was re-estimated with the shortened time series (Fig. 4.3).

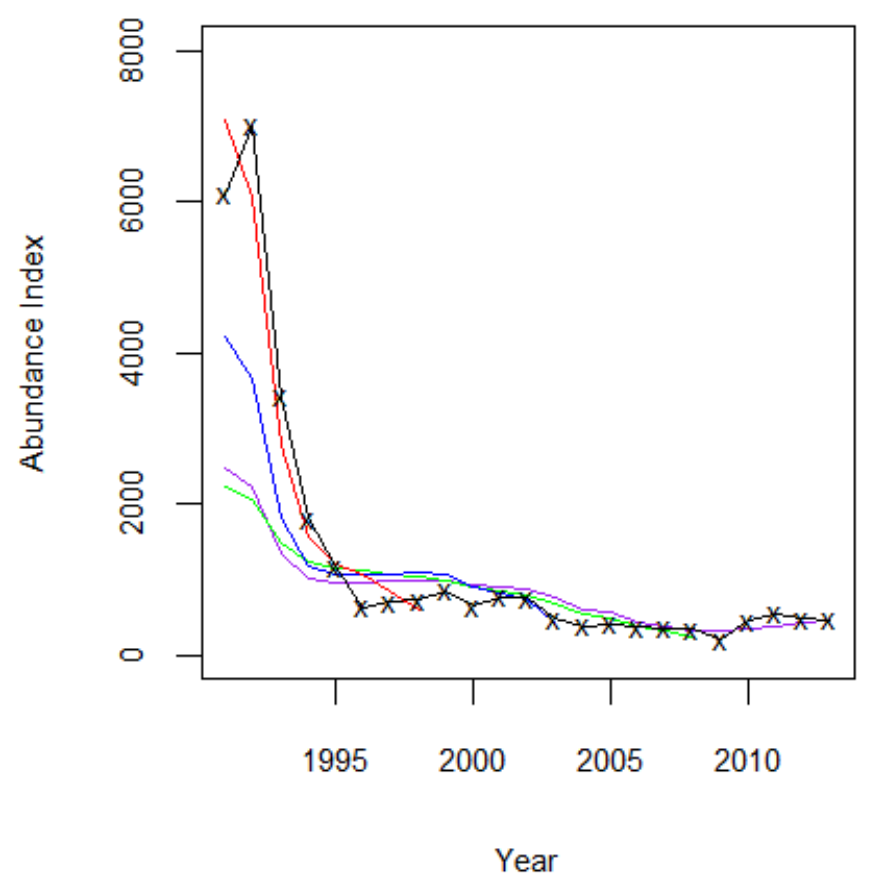

Figure 4.3: Retrospective fit of the Closed Population Model of the Andes Complex orange roughy fishery. The observed abundance index (black) is compared to the closed population model with four different lengths: the full dataset (purple); 5 years removed (green); 10 years removed (blue); and 15 years removed (red).

The retrospective fit showed an improved fit to the start of the fishery when the length of the time series was reduced, with the $K$ being the smallest possible that still allows the catch to be taken (Clark and Dunn, 
2012) (Fig. 4.3).

\subsubsection{The Changing Growth Model}

This hypothesis allowed the population growth rate of the Andes Complex fishery to change at one point in the time series (Eq. 2.8). The transition year $t^{*}$ between growth rates was selected by offering the model a range of possible years and selecting the one with the smallest negative log-likelihood. The transition point between growth rates that minimised the log-likelihood was at $t^{*}=6$ years (Tab. 4.2). The first population growth rate $r 1$ was estimated at approximately zero and the second growth rate $r 2$ had a reasonably high estimate (Tab. 4.3), relative to the Closed Population Model.

Table 4.2: The likelihood values for a range of transition years between growth rates

\begin{tabular}{cc}
$t^{*}$ & Likelihood \\
\hline 4 & 8.31 \\
5 & 4.58 \\
6 & 2.73 \\
7 & 8.22 \\
8 & 5.32 \\
9 & 7.31 \\
10 & 9.17
\end{tabular}

The parameter estimate for $\mathrm{K}$ from the Changing Growth Model (Tab. 4.3) was similar to the estimate from the Closed Population Model, although the confidence interval was more precise. The estimate for $r 1$ means the population did not grow until the transition point $t^{*}$, then the growth rate was higher than the estimate of $r$ from the Closed Population Model. 
Table 4.3: Parameter estimates and their corresponding 95\% confidence intervals for the Changing Growth Model

\begin{tabular}{llll} 
Parameter & Estimate & Lower & Upper \\
\hline$K$ & 20062 & 18834 & 21029 \\
$r 1$ & 0 & 0.0000 & 0.1026 \\
$r 2$ & 0.5911 & 0.4545 & 0.6878 \\
$q$ & 0.2451 & 0.1891 & 0.2923 \\
$\sigma$ & 0.2654 & 0.1842 & 0.3079
\end{tabular}

The Changing Growth Model struggled to fit the initial decline in the fishery, but after 1994 it provided a good visual fit to the observed Andes orange roughy abundance index (Fig. 4.4). The confidence intervals around $\hat{I}_{t}$ widened considerably after $t^{*}$ as the population started to grow. The standardised residuals showed a relatively random scatter after 1994, although they were consistently below the 0 line from 2003-2006 (Fig. 4.4). 

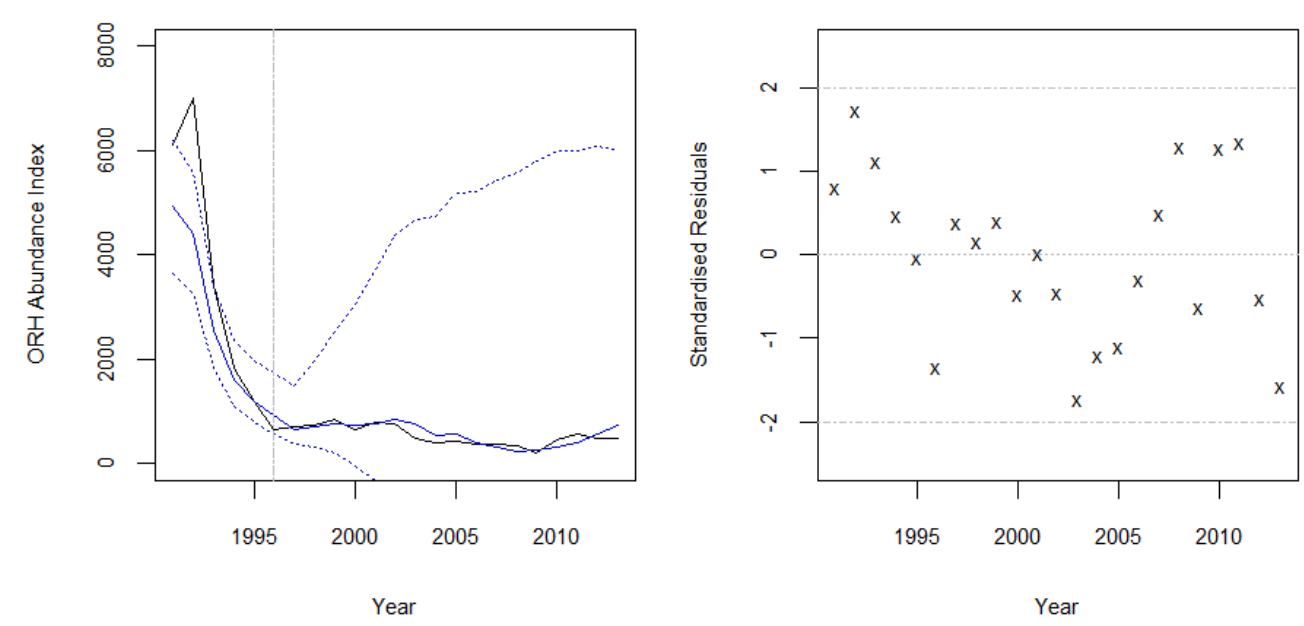

Figure 4.4: Estimated Andes orange roughy population index (blue) with corresponding $95 \%$ confidence intervals for the Changing Growth Model and the observed population index (black) from 1991 - 2013. The vertical dashed line indicates the transition point between growth rates. The residual plot shows the difference between the estimated and observed index, the central dashed line indicates no difference (zero) and the periphery dashed lines indicate $+/-2$ standard deviations of the residuals.

\subsubsection{The Altered Environment Model}

\section{The Altered Environment Model ( $r$ )}

The Altered Environment Model $(r)$ allowed for the population growth rate to change annually, based on an observed change in environmental conditions. The parameter estimate for $K$ (Tab. 4.4) was similar to the Closed Population Model (Fig. 4.6). 

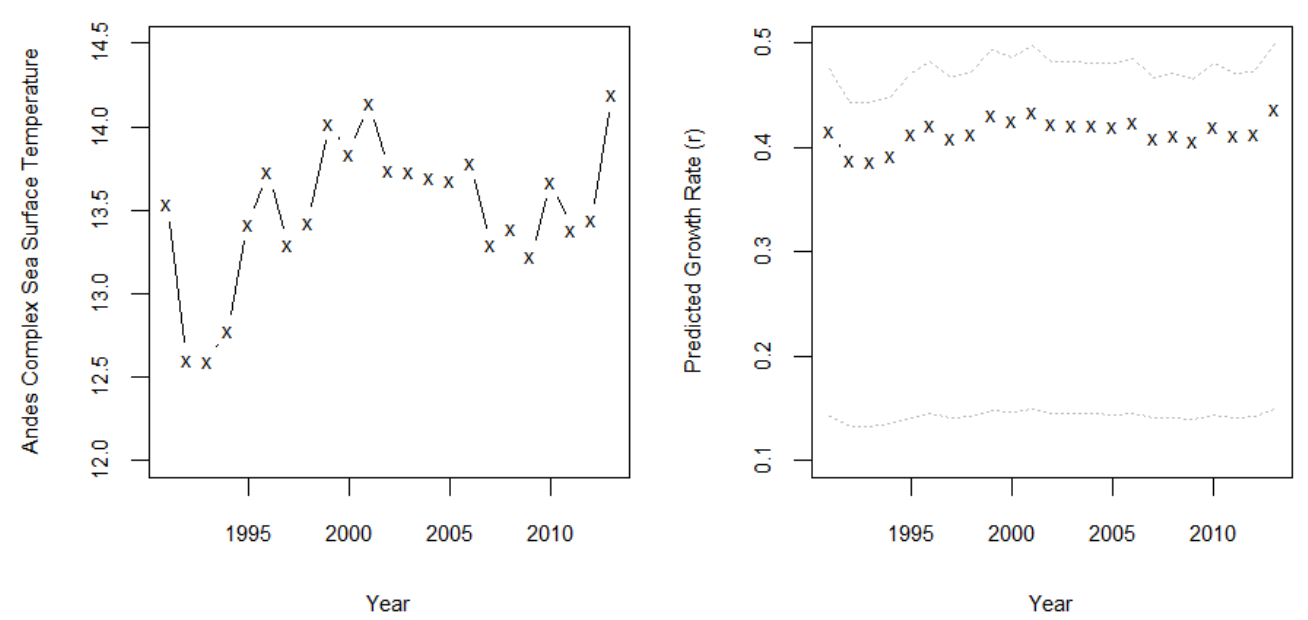

Figure 4.5: The mean annual sea surface temperature values for the Andes Complex and the corresponding values of growth rate $r$ and $95 \%$ confidence intervals generated by using the parameter estimates of $m$ and $c$ from the Altered Environment Model $(r)$.

SST showed a slight increase over the time series, with the majority of values around $13.5^{\circ} \mathrm{C}$ (Fig. 4.7). The variability in the temperature was much lower than that of the fishery. The estimate of $r$ (Fig. 4.7) was $\approx 0.4$, which was also similar to the estimate from the null model. The additional information conveyed by my environmental vector has not influenced my results. The parameter estimate for $c(0)$ made a negligible contribution to $r$.

Table 4.4: Parameter estimates and their corresponding 95\% confidence intervals for the Altered Environment Model $(r)$

\begin{tabular}{llll} 
Parameter & Estimate & Lower & Upper \\
\hline$K$ & 15864 & 15009 & 24977 \\
$m$ & 0.0307 & 0.0106 & 0.0350 \\
$c$ & 0.0000 & 0.0000 & 0.0026 \\
$q$ & 0.204 & 0.0844 & 0.2394 \\
$\sigma$ & 0.432 & 0.2844 & 0.5120
\end{tabular}

This model could not fit the initial rapid decline of the time series (Fig. 
4.6). From 1996 untill 2005, the Altered Environment Model ( $r$ ) overestimated the biomass and from 2006 until 2010, the model fitted the observed index well. This model struggled to fit the finer movements of the observed abundance index $I_{t}$. This was evident as the residual pattern moved above and below 0 sequentially (Fig. 4.6). The residuals for the 1992 and 1993 were outside 2 standard deviations.
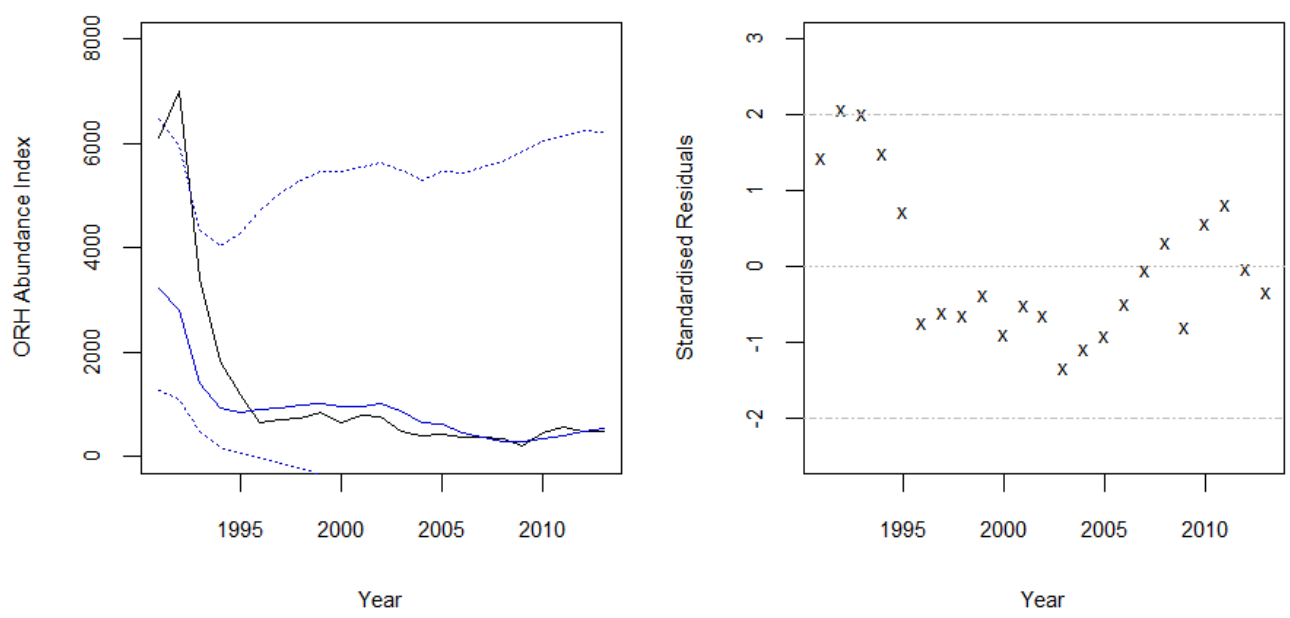

Figure 4.6: Estimated Andes orange roughy population index (blue) with corresponding 95\% confidence intervals for the Altered Environment Model $(r)$ and the observed population index (black) from 1991 - 2013. The residual plot shows the difference between the estimated and observed index; the central dashed line indicates no difference (zero) and the periphery dashed lines indicate $+/-3$ standard deviations of the residuals.

\section{The Altered Environment Model (K)}

The vector of SST gradually increased until 2000, then stabilised around $13.5^{\circ} \mathrm{C}$ for the rest of the time series (Fig. 4.7). This trend was in contrast to the abundance index, which had the opposite trajectory. Considering this, 
the poor fit of the Altered Environment Model $(r)$ was unsurprising. The SST vector was applied to $K$ because it provided more leverage.
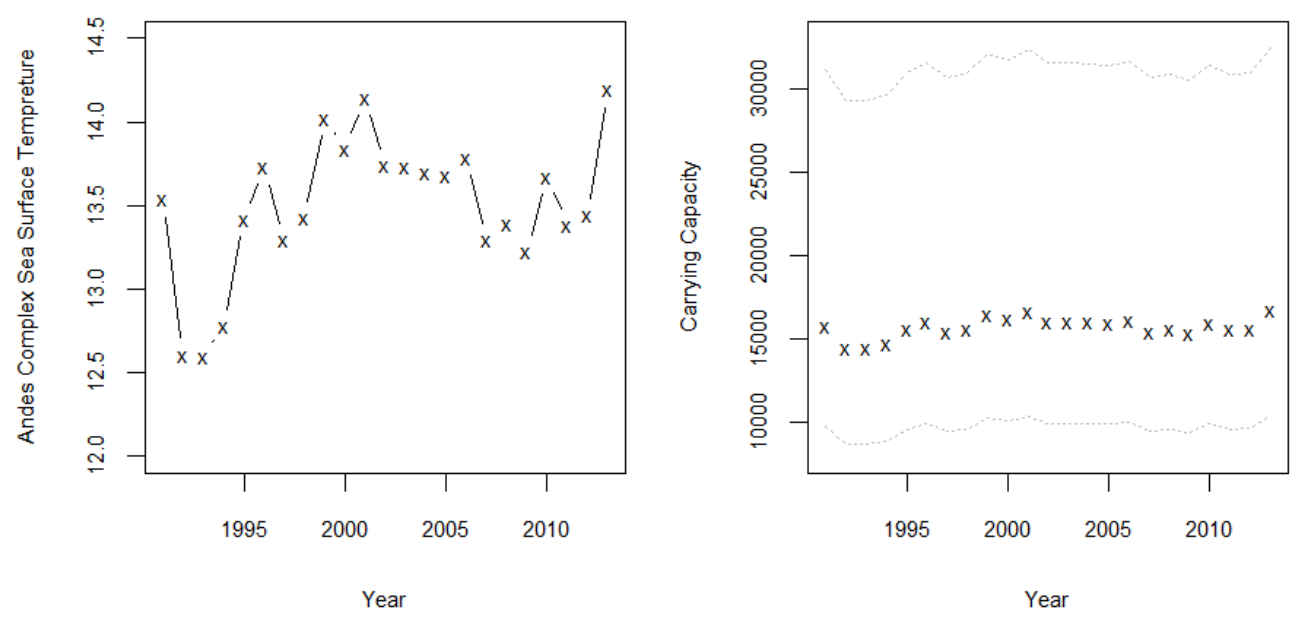

Figure 4.7: The mean annual sea surface temperature values for the Andes Complex and the corresponding values of carrying capacity $K$ and its $95 \%$ confidence interval, generated by using the parameter estimates of $m$ and $c$ from the Altered Environment Model $(K)$.

The parameter estimates for $r$ and the $K$ were again similar to the Closed Population Model (Tab. 4.5). As the intervals around $m$ and $c$ were imprecise, this indicated that the vector of SST values was not informative for predicting the Andes Complex carrying capacity $K$.

Table 4.5: Parameter estimates and their corresponding 95\% confidence intervals for the Altered Environment Model $(K)$

\begin{tabular}{llll} 
Parameter & Estimate & Lower & Upper \\
\hline$m$ & 1345 & 987 & 1936 \\
$c$ & -2000 & -5000 & 10000 \\
$r$ & 0.4229 & 0.1732 & 0.4673 \\
$q$ & 0.0692 & 0.0961 & 0.2818 \\
$\sigma$ & 0.2583 & 0.2793 & 0.5168
\end{tabular}


The imprecise parameter estimates had corresponding large confidence intervals (Fig. 4.8). The fit of the estimated index was similar to the Altered Environment Model $(r)$ but the precision of the estimates has increased drastically. The Altered Environment Model $(K)$ struggled to fit the time series from 1991 to 1995, then overestimated the abundance till 2007 and provided a good fit thereafter (Fig. 4.8). The distinct pattern in the residuals was similar to the Altered Environment Model $(r)$ and indicated a poor model fit.
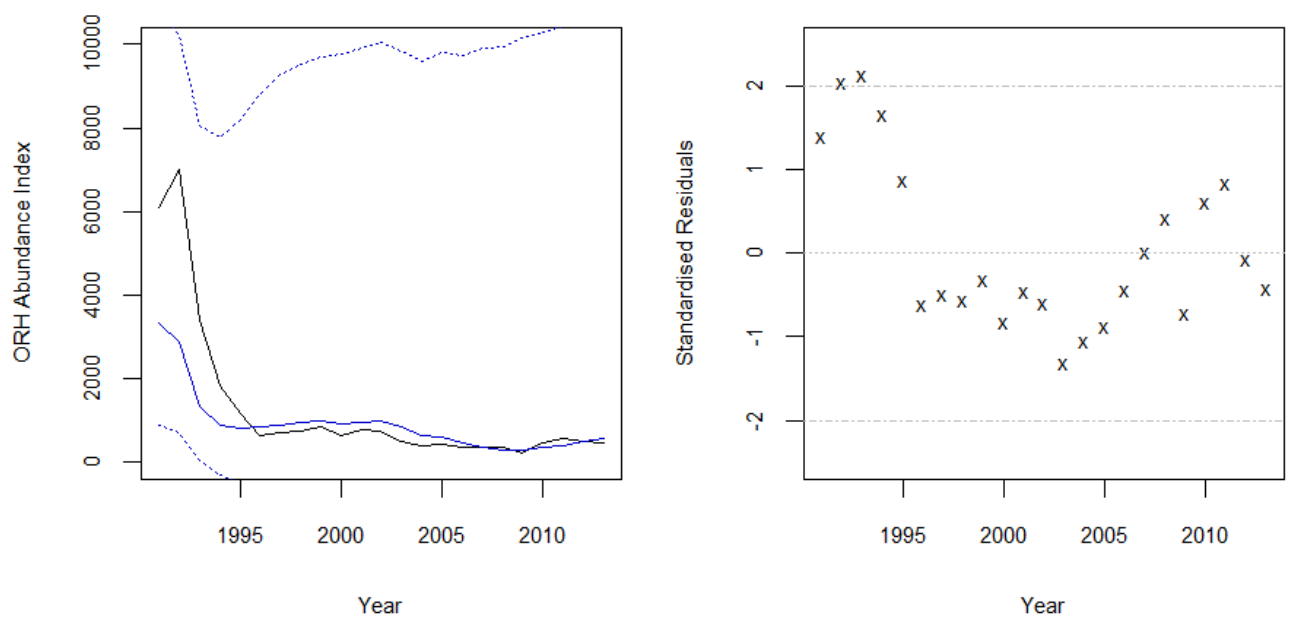

Figure 4.8: Estimated Andes orange roughy population index (blue) with corresponding 95\% confidence intervals for the Altered Environment Model $(K)$ and the observed population index (black) from 1991 - 2013. The residual plot shows the difference between the estimated and observed index, the central dashed line indicates no difference (zero) and the periphery dashed lines indicate $+/-3$ standard deviations of the residuals. 


\subsubsection{The Disturbance Model}

The Disturbance Model parameter estimates were close to starting values for both $r$ and $K$ (Tab. 4.6). The estimate of $K$ was higher than the Closed Population Model and the estimate of $r$ was lower. The confidence intervals around $r$ were imprecise, this led to the inflated confidence intervals evident in figure 4.9 .

Table 4.6: Parameter estimates and their corresponding 95\% confidence intervals for the Disturbance Model, $*$ indicates the estimate has hit a bound.

\begin{tabular}{llll} 
Parameter & Estimate & Lower & Upper \\
\hline$K$ & 25380 & 15970 & 42144 \\
$r$ & 0.1325 & 0.0001 & 0.3988 \\
$q$ & 88.76 & 38.10 & 197.94 \\
$\sigma$ & 0.4470 & 0.4146 & 0.7380
\end{tabular}

The Disturbance Model went some way to fitting the initial decline in the fishery, with a steep decline evident from 1991 to 1994 (Fig. 4.9). However, the estimated decline in $\hat{I}_{t}$ occured earlier and faster than in $I_{t}$. From 1995 to 2004, the Disturbance Model overestimated the index and from 2004 onwards, it provided a good fit (Fig. 4.9). 

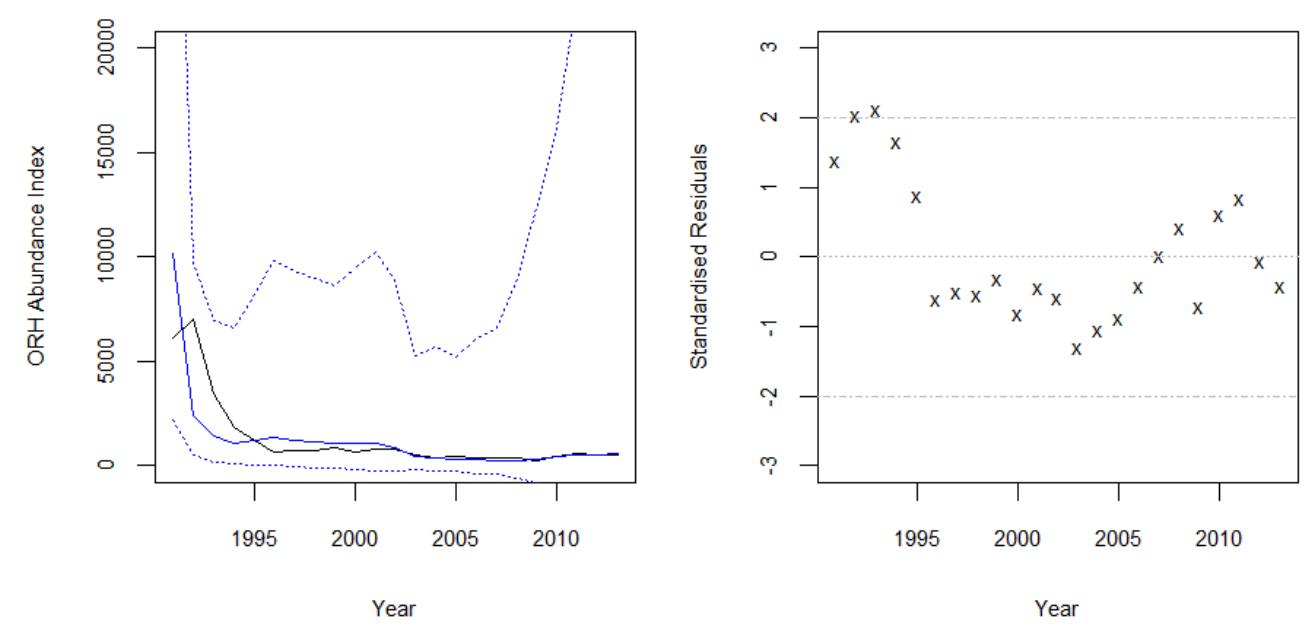

Figure 4.9: Estimated Andes orange roughy population index (blue) with corresponding $95 \%$ confidence intervals for the Disturbance Model and the observed population index (black) from 1991 - 2013. The residual plot shows the difference between the estimated and observed index; the central dashed line indicates no difference (zero) and the periphery dashed lines indicate $+/-3$ standard deviations of the residuals.

The number of tows on the Andes Complex fluctuated until 1995, then $n$ steadily increased to a peak in 2002 (Fig. 4.10). Since 2007, there has been a substantial reduction in fishing effort at the Andes Complex. The largest fluctuations in fishing effort occurred when the observed abundance index was relatively stable. This uncoupling in trends led to the inflation in confidence intervals around $\hat{I}_{t}$ from 2007. The disturbance index followed a similar trajectory to the number of tows at the Andes Complex but because the tows are cumulative in the disturbance index, it has a three year lag. 

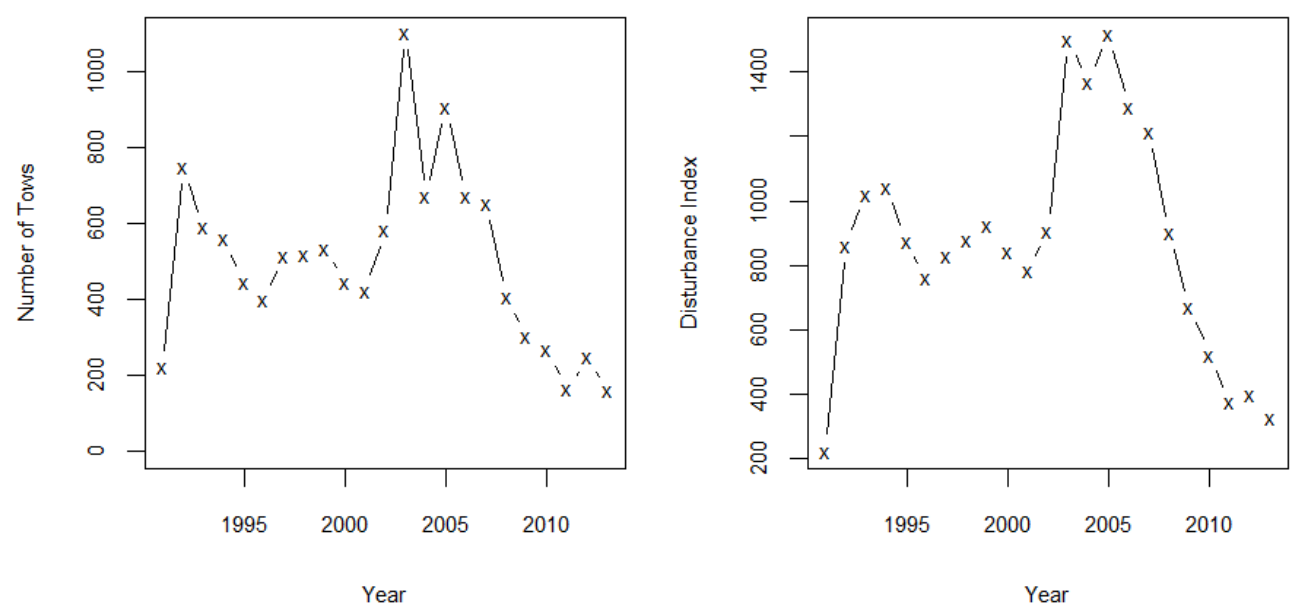

Figure 4.10: The annual number of tows at the Andes Complex (left) and the disturbance index that was applied to the carrying capacity (right), from 1991 to 2013.

\subsubsection{The Habitat Degradation Model}

The Habitat Degradation Model scaled the carrying capacity $K$ by a vector of habitat damage. Both vectors of habitat damage increased quickly then stabilised around their respective maxima (Fig. 4.11). This indicates most of the habitat damage was done in the first $\sim 10$ years of the fishery, with little or none thereafter. The weighted habitat damage vector reached its asymptote quicker, although the overall damage attained was less than the unweighted habitat damage vector (Fig. 4.11). 

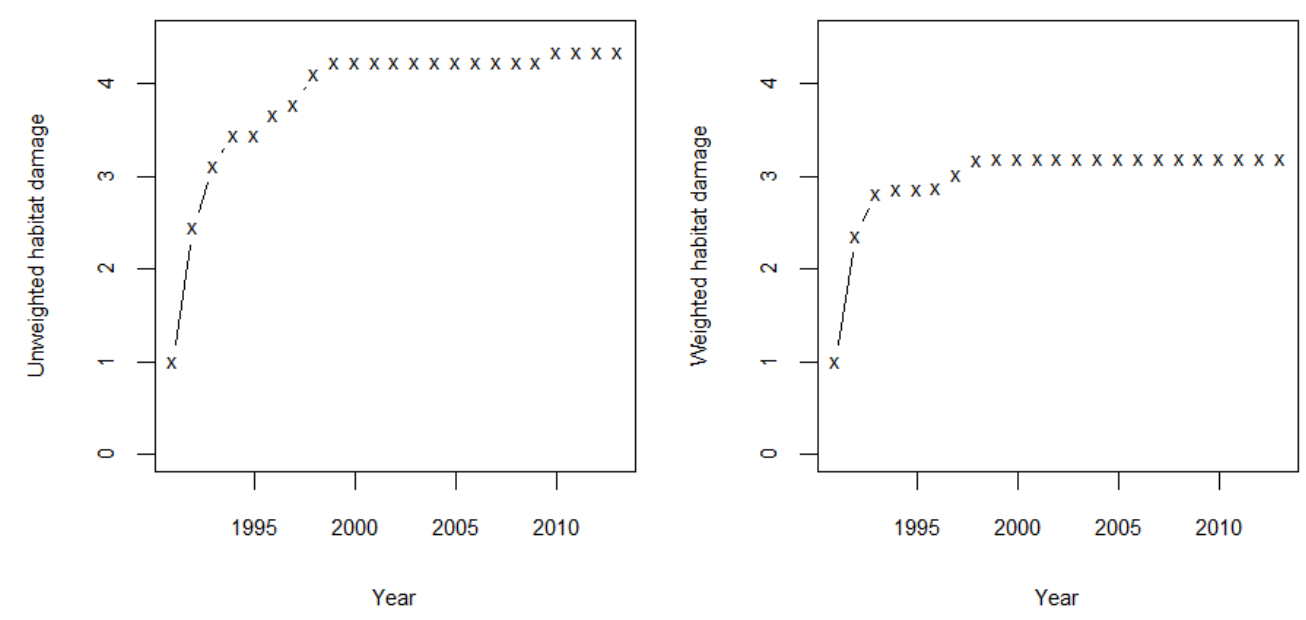

Figure 4.11: Vectors of unweighted and weighted habitat damage for the Andes Complex. Unweighted habitat damage assumes all the grid has equal value, whereas the weighted habitat gives grid cells with seamounts an order of magnitude more value.

\section{Unweighted Habitat Degradation Model}

The Unweighted Habitat Degradation Model parameter estimates for $K$ and $r$ were larger than the Closed Population Model (Tab. 4.8). The precision in the estimates around parameter values were similar to the Closed Population Model.

Table 4.7: Parameter estimates and their corresponding 95\% confidence intervals for the Unweighted Habitat Model

\begin{tabular}{llll} 
Parameter & Estimate & Lower & Upper \\
\hline$K$ & 51513 & 44222 & 70130 \\
$r$ & 0.3085 & 0.1498 & 0.4163 \\
$q$ & 0.0871 & 0.0447 & 0.1308 \\
$\sigma$ & 0.2835 & 0.1799 & 0.3331
\end{tabular}

The model provided a good fit to the observed abundance index. It went some way to fitting the initial decline (Fig. 4.12). A point of differ- 
ence for this model was that the rapid decline of the observed index was within the confidence intervals for the predicted index, for 1991 and 1992 at least. From 1995 to 2000, the Unweighted Habitat Degradation Model underestimated the observed index but after 1999, it provided an excellent fit, with the standardised residuals scattered around zero (Fig. 4.12). The observed abundance index was within the confidence intervals of the Unweighted Habitat Model. Although other models achieved this, their confidence intervals were considerably less precise.
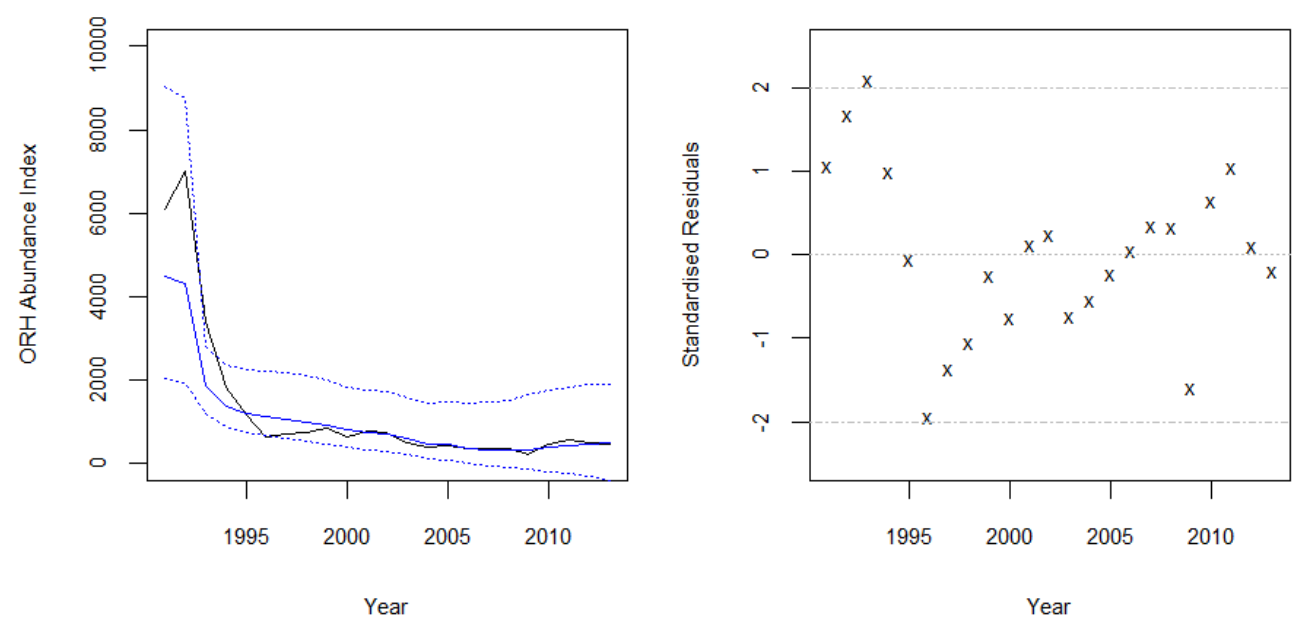

Figure 4.12: Estimated Andes orange roughy population index (blue) with corresponding 95\% confidence intervals for the Unweighted Habitat Degradation Model and the observed population index (black) from 1991 - 2013. The residual plot shows the difference between the estimated and observed index; the central dashed line indicates no difference (zero) and the periphery dashed lines indicate $+/-2$ standard deviations of the residuals. 


\section{Transformed Habitat Degradation Model}

The parameter estimates for the Transformed Habitat Degradation Model are displayed in table 4.8. The estimates for $K$ for the Habitat Degradation Models were substantially higher then the other models, as $K$ was inflated by the habitat degradation $H D$ vector (Eq. 2.12). The parameter estimate for $r$ in the Transformed Habitat Degradation Model was lower than the Unweighted Habitat Degradation Model and comparable to the Closed Population Model.

Table 4.8: Parameter estimates and their corresponding $95 \%$ confidence intervals for the Transformed Habitat Degradation Model

\begin{tabular}{llll} 
Parameter & Estimate & Lower & Upper \\
\hline$K$ & 52863 & 43245 & 66440 \\
$r$ & 0.2434 & 0.0995 & 0.4073 \\
$q$ & 0.0463 & 0.0470 & 0.1654 \\
$\sigma$ & 0.3312 & 0.2006 & 0.3962
\end{tabular}

The log-transformation of the habitat degradation vector was undertaken in an effort to help the model fit the initial decline of the index, after a non-linear relationship between habitat degradation and time was suspected (Fig. 4.11). The Transformed Habitat Degradation Model struggled to fit the initial decline in the index. The decline was estimated to be earlier than in the Unweighted Transformed Habitat Degradation Model (Fig. 4.13). The standardised residuals illustrate the model underestimated the orange roughy abundance until 1994, then overestimated abundance until 1999, thereafter it provided an acceptable fit (Fig. 4.13). The key difference between this model and the Unweighted Habitat Degradation Model was that the initial decline in $I_{t}$ was just not within the $95 \%$ confidence interval (Fig. 4.13). 

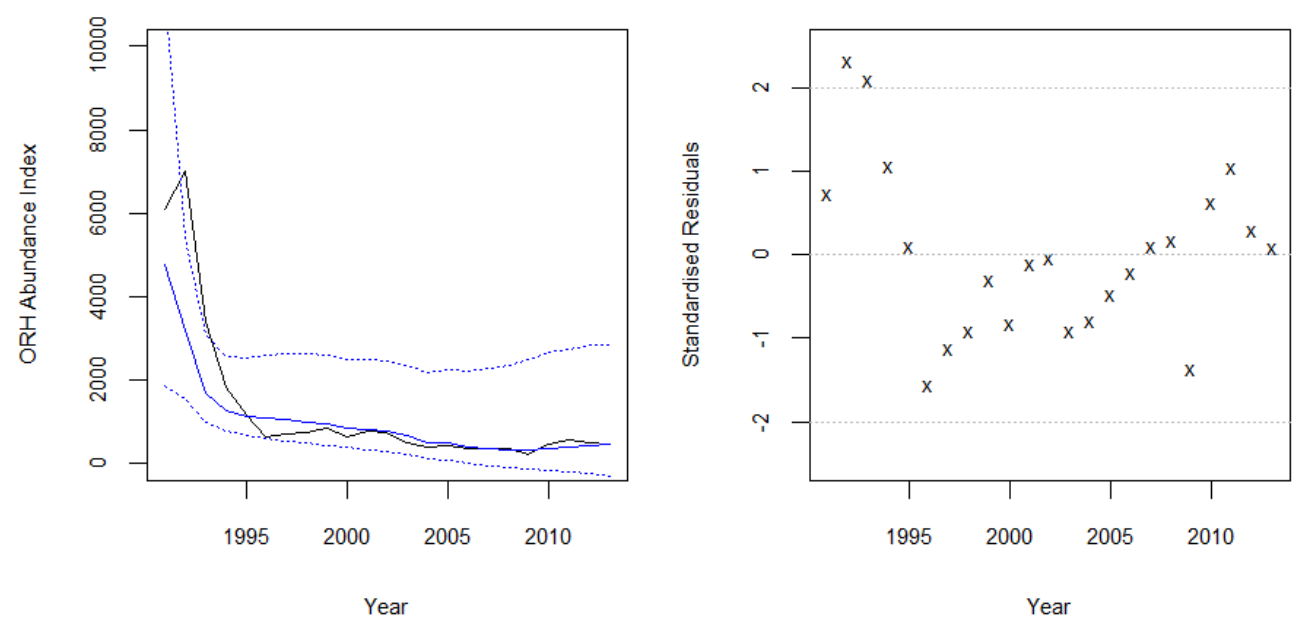

Figure 4.13: Estimated Andes orange roughy population index (blue) with corresponding 95\% confidence intervals for the Transformed Habitat Degradation Model and the observed population index (black) from $1991-2013$. The residual plot shows the difference between the estimated and observed index; the central dashed line indicates no difference (zero) and the periphery dashed lines indicate $+/-2$ standard deviations of the residuals.

\section{Weighted Habitat Degradation}

The Weighted Habitat Degradation Model put a higher weight on cells of the Andes Complex that have seamounts within them. This model predicted a lower carrying capacity than the other Habitat Degradation Models (Tab. 4.9).

Table 4.9: Parameter estimates and their corresponding 95\% confidence intervals for the Weighted Habitat Degradation Model

\begin{tabular}{llll} 
Parameter & Estimate & Lower & Upper \\
\hline$K$ & 40302 & 36167 & 52502 \\
$r$ & 0.3091 & 0.1512 & 0.4137 \\
$q$ & 0.1041 & 0.0576 & 0.1520 \\
$\sigma$ & 0.3141 & 0.2079 & 0.3728
\end{tabular}


The Weighted Habitat Degradation Model did not fit the initial decline in the observed index (Fig. 4.14). The residual plot showed that from 1991 - 1994 the Weighted Habitat Degradation Model underestimated the index, and from 1995 - 2006 it overestimated the index (Fig. 4.14). The 95\% confidence intervals did not include the observed initial decline in the orange roughy fishery, although they came close.
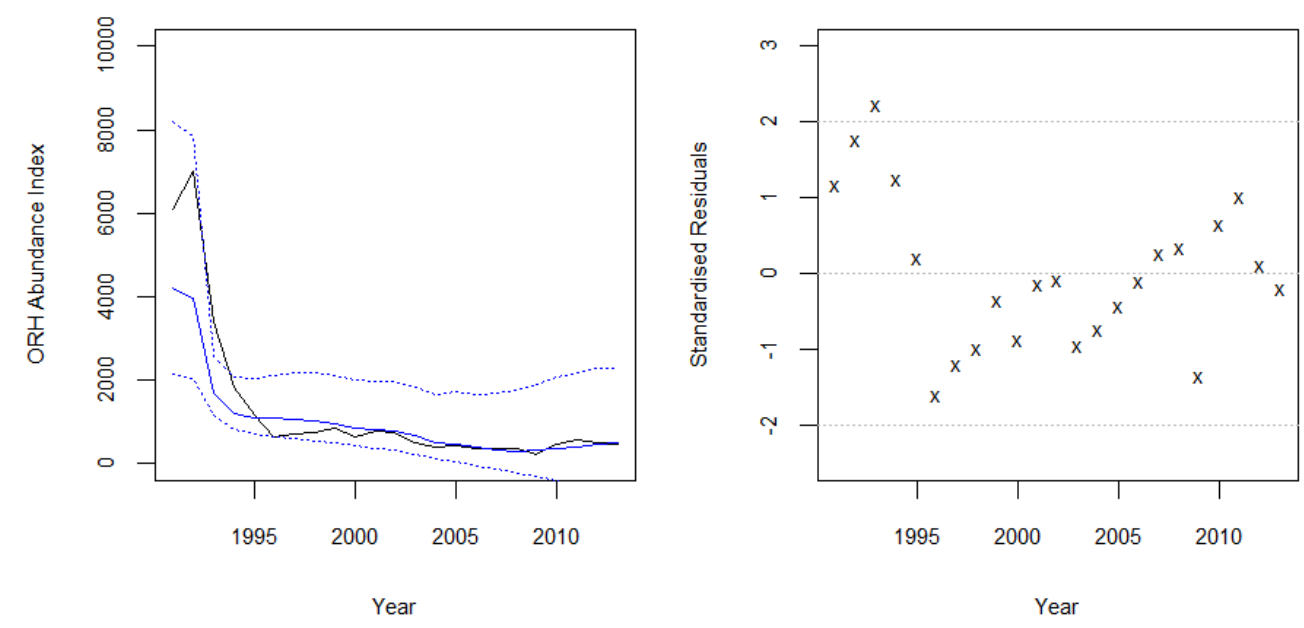

Figure 4.14: Estimated Andes orange roughy population index (blue) with corresponding $95 \%$ confidence intervals for the Weighted Habitat Degradation Model and the observed population index (black) from 1991 - 2013. The residual plot shows the difference between the estimated and observed index; the central dashed line indicates no difference (zero) and the periphery dashed lines indicate $+/-3$ standard deviations of the residuals.

\subsection{Model Evaluation}

The final model evaluation table (Tab. 4.10) summarised each candidate model with a spectrum of model fit statistics. The model that had the 
lowest value for the negative log-likelihood was the Unweighted Habitat Degradation Model. However, log-likelihood alone was not satisfactory, as it did not account for degrees of freedom. The $A I C_{c}$ and $B I C$, provided a more robust measure of model fit. Two models had comparable values for $A I C_{c}$ and $B I C$; the Changing Growth Model and the Unweighted Habitat Degradation Model. The Changing Growth Model had six unknown parameters and the Unweighted Habitat Degradation Model had five unknown parameters (Tab. 4.10). The Unweighted Habitat Degradation Model had additional information about habitat degradation that had inherent assumptions about the value of habitat within each cell of the Andes Complex, which my model evaluation did not account for. The Unweighted Degradation Habitat Model was one of three alternates examining the same hypothesis. In this analysis the unweighted model provided the best fit but all three alternate models had credible fits.

Table 4.10: Model Evaluation Table: each model with its corresponding Likelihood, Number of Parameters, AIC, AIC_c, BIC and MSY

\begin{tabular}{llllll} 
Model & -log-Likelihood & Unknown Parameters & AIC & AIC_c & BIC \\
\hline Closed Population Model & 13.15 & 4 & 34.30 & 36.52 & 38.84 \\
Changing Growth Model & 1.702 & 6 & 15.32 & 20.57 & 22.14 \\
Altered Environment Model $(r)$ & 13.32 & 5 & 42.31 & 40.17 & 42.31 \\
Altered Environment Model $(K)$ & 13.04 & 5 & 36.07 & 39.60 & 41.74 \\
Disturbance Model & 14.12 & 4 & 36.23 & 38.45 & 40.77 \\
Unweighted Habitat & 3.65 & 4 & 15.29 & 17.52 & 19.83 \\
Transformed Habitat & 7.22 & 4 & 22.44 & 24.66 & 26.98 \\
Weighted Habitat & 6.00 & 4 & 20.00 & 22.23 & 24.55
\end{tabular}



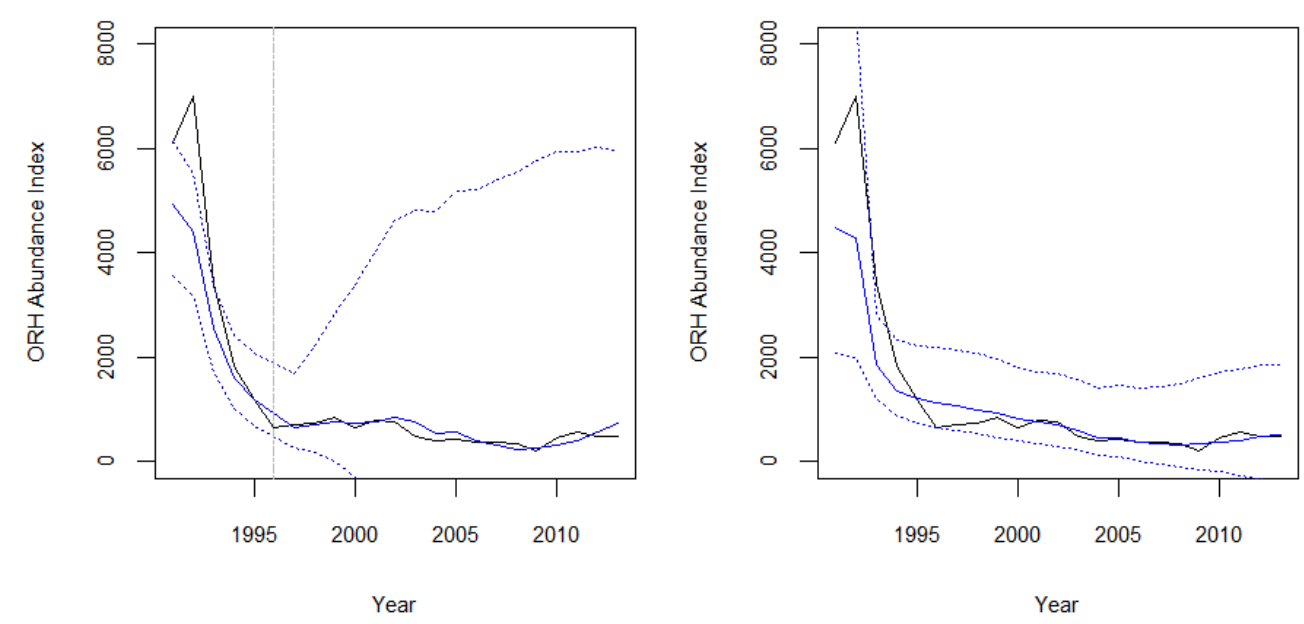

Figure 4.15: A comparison of the observed (black) and expected (blue) abundance indicies of the two best candidate models for the Andes Complex orange roughy fishery, the Changing Growth Model (left) and the Unweighted Habitat Degradation Model (right). Both models are plotted with their corresponding $95 \%$ confidence intervals (dashed lines) and the Changing Growth Model has a vertical line corresponding to the transition point between the two growth rates.

The two best candidate model fits were compared in figure 4.15 (their profiles are in Appendix 6.2). Both models failed to fit the initial decline of the observed index. The Unweighted Habitat Degradation Model had the initial decline within its $95 \%$ confidence interval. The Unweighted Habitat Degradation Model overestimated the observed index from 1995 and 2001, a feature in many of the candidate models, although the observed index was still within the confidence interval. Overall, the Changing Growth Model provided the best fit to the abundance index, with no residuals outside of 2 standard deviations. However, its additional parameters discredited it from being the best candidate model. The Unweighted Habitat Degradation Model had the lowest log-likelihood, the lowest $B I C$, and it 
provided the best overall model fit. Therefore, the Unweighted Habitat Degradation Model was the best candidate model to explain the Andes Complex orange roughy abundance. 


\section{Chapter 5}

\section{Discussion}

This thesis examined a range of hypotheses about drivers of the potential hyperdepletion relationship evident in the abundance index of the Andes Complex orange roughy stock. The hypotheses tested here kept a closed population, and a linear relationship between stock biomass and the abundance index, and then assumed the apparently non-linear trend was caused by a changing population or exogenous factors. The hypotheses that provided the best model fits to my observed abundance index were the Changing Growth Model, and a Habitat Degradation Model. Both of these models fitted the data better than the null model, which assumed a simple closed population unaffected by any exogenous factors. I hypothesised that a change in the environmental conditions at the Andes Complex, namely SST, had the potential to influence the orange roughy population, but found no evidence to support this hypothesis. I also examined the effect of fishing disturbance on the Andes Complex orange roughy populations. The disturbance hypothesis led to a rapid population decline, but this model was least supported by the data, over an an- 
nual time series.

The thorough data screening, conducted prior to modeling, was in more depth than a typical fisheries management analysis. This process in essence standardised the dataset and removed a lot of the variability prior to modeling. The results from the GLM did not unduly influence the abundance indices, with little difference between the GLM predicted indices and the raw unstandardised indices.

The null model, a standard Schaefer SPM for a closed population, displayed a poor fit to the observed abundance trend. This fit was anticipated, given a poor fit had been observed in previous assessments using age structured models (Dunn, 2006; Clark and Dunn, 2012). The null model tells me that closed population demographics cannot easily explain the trends in population abundance at the Andes Complex. Therefore, the Andes Complex should not be considered a closed population and is either a component of a wider ECR orange roughy population or the parameters of the stock are not constant over time.

The change in intrinsic growth rate predicted by the Changing Growth Model $(0 \rightarrow 0.65)$ would be unprecedented. A change in production within a population is unlikely to exceed an order of magnitude. Realistic growth rates for orange roughy are low. Butterworth and Brandao (2005) estimated orange roughy $r$ at 0.08 in accordance with MSY being $0.3 B_{0}$, which might be an over estimate according to Francis and Clark (2005). Growth rates of more productive species, for example, Atlantic Cod (Gadus morhua) which mature at $2-4$ years compared to $20-30$ years for orange roughy, are in the vicinity of $r=0.18$ (Myers et al., 1997). Therefore, the second estimated growth rate is likely to be unrealistic. This 
supports the assertion that the Andes Complex cannot be demographically closed: the population must be supplemented by external immigration from the wider ECR orange roughy population to enable the observed trends in abundance.

The critique of all candidate models was that they struggled to fit the rapid decline of the Andes Complex orange roughy CPUE. This decline cannot be explained in terms of the weight of catch removed, given the estimated carrying capacity (Dunn, 2006). The Andes fishery displayed two contrasting trajectories, modeling these trajectories as two separate populations, or alternatively applying two of the hypothesised models in tandem, has the potential to enable a good fit to the initial decline. Analogous declines are a feature in New Zealand fisheries for orange roughy (Dunn, 2011), in other New Zealand species (Field and Clark, 2001; Harley et al., 2001) and in other fisheries around the world (Walters and Maguire, 1996; Wiff et al., 2012). This trend in CPUE is called hyperdepletion (Harley et al., 2001), and is often attributed to ignoring the spatial structure of a fishery (Walters, 2003). Here I investigated spatial structuring but found no differences between my abundance indicies of different spatial strata. This suggests hyperdepletion in the Andes fishery is not spatially driven. However, migration has been ignored, and has the potential to be a explanation of the hyperdepletion relationship (Dunn and Forman, 2011). Stock assessment becomes difficult with a hyperdepletion abundance index, as a consequence of an abundance trend which is troublesome to fit with a single area model. Modeling multiple areas requires more data and assumptions but still may encounter problems, for example, Dunn \& An- 
derson (2012). Fisheries stock assessment scientists are cautious about the use of CPUE data, because of potential biases and non-linear relationships between biomass and population abundance (Dunn et al., 2000). Shorter time series can be modeled successfully using traditional methods. This was demonstrated in my retrospective fit, and by Clark \& Dunn (2012). The Changing Growth Model provided a very simple explanation for the initial decline in the observed abundance index. The Andes Complex was pristine, at carrying capacity prior to the start of the fishery, and as such, there was no net population growth. Once the fishery began, population growth was zero, implying it did not grow immediately. A potential mechanism behind this lag is a threshold population density, which once surpassed, stimulates immigration to commence. The Habitat Degradation Model offers an alternate explanation; once an area was fished, there was a reduction in habitat quality, and as a consequence, it supported less fish. Most of the area of the Andes Complex was fished in the first five years of the time series, so the reduction in carrying capacity occurred quickly.

The Andes Complex hills provide an exposed area of hard substrate that supports an array of macro-invertebrates called "biogenic habitat", which add habitat complexity (Etnoyer, 2009; Ministry for Primary Industries, 2014). These benthic invertebrates are generally large, sessile, fragile and slow growing; as a consequence, they are highly vulnerable to the effects of bottom trawling (Clark, 2010; Rowden et al., 2010; Ministry for Primary Industries, 2014). Seamount fisheries are generally high intensity, with numerous short tows that target a small area (Clark, 2010). In developing fisheries coral by-catch initially forms a high proportion of the catch 
(up to $1 / 3$ (Anderson and Clark, 2003)). As a consequence, fishers display fidelity to established areas and towlines to avoid damaging gear (Clark, 2010). Once the benthic substrate is scraped clear, then the fishery can continue unhindered, without invertebrate by-catch and gear damage. Another seamount complex on the CR, the Graveyard Complex, provides a salient example of the change in macro-invertebrate assemblage, as a consequence of bottom trawling. Clark \& Rowden (2009) showed unfished hills within the complex had a greater macro-invertebrate diversity and density. Niklitschek et al. (2010) examined a developing seamount fishery in Chile, and observed a higher orange roughy and by-catch species densities in unexploited areas, compared to areas that had already sustained bottom trawls. How orange roughy interact with the wider ecosystem is poorly understood (Dunn and Forman, 2011). Roughy have been demonstrated to increase tilt angle and dive in response to disturbance (Koslow et al., 1995; O'Driscoll and Joux, 2012). This diving response could be to find refuge amongst coral from larger predators known to predate roughy, for example, toothed whales (Dunn and Devine, 2010; Koslow et al., 1995). Seamounts are a delicately balanced trophic web (Pitcher et al., 2010).

I have used a dataset that came from the commercial fishing fleet, and this inherently comes with a suite of potential issues. The dataset was treated as if it was a random sample across all the spatial strata of the Andes Complex but it was not, as the commercial fleet targeted roughy aggregations. They did not fish areas where no roughy were detected, for example, the flat areas between seamounts. Unfished strata are unlikely to hold significant amounts of orange roughy biomass, but they should 
be considered (Walters, 2003). Unfished strata are not indicative of an absence of orange roughy, just a population density that is not commercially viable. Even if the population density of the unfished strata is low, the cummulative size of these strata may constitute a significant portion of the population. I assumed that tows were independent of one another but in fact there will be some auto-correlation within the fishing patterns of each vessel through time (Nishida and Chen, 2004). Fishers showed fidelity to established towlines, therefore, tows were not independent of one and other. I assumed the efficiency of the fleet was homogenous over time, but this is unlikely to be true since a certain amount of learning is required to effectively fish each individual seamount (Maunder et al., 2006).

My catch data only conveyed information about the section of the population vulnerable to fishing. This is determined by the gear selectivity, size and age of the fish (Maunder et al., 2006). The Andes Complex orange roughy fishery was treated as if it had no density dependent population demographics. Two parameters are likely to be influenced by changes in population density: the population growth rate and the catchability coefficient (Walters and Maguire, 1996; Wilberg et al., 2009). My SPM parameterises a density dependent change in growth but I do not consider density dependent catchability. As population abundance declined, the fishery will sustain spatial contractions, and as a consequence, catchability will vary. This is thought to be one of the contributors to the collapse of the Atlantic Cod fishery (Maunder and Punt, 2004; Wilberg et al., 2009). The Andes orange roughy population will have some form of age and size structure (Clark, 2001). Knowledge of this would provide a biological insight into the status of the stock and help develop appropriate estimates 
of unknown population demographics. However, size structure has remained constant in other exploited orange roughy populations on the $C R$ (Clark, 2001).

There were assumptions in some hypothesised models that my model selection did not take into account. An example is the Habitat Degradation Model, where I have made assumptions about the value of the habitat within each grid cell of the Andes Complex. It is important to recognise and consider these assumptions, as they convey uncertainty about my parameter estimates that would be otherwise unrecognised. An alternate approach would be to structure my model in such a way that parameterised and estimated this relationship. For my Habitat Degradation Model, this would mean letting the model determine the value of the habitat weight within each cell. In this remodeled scenario, my model selection statistics (e.g. AIC) would account for this previously unknown information and penalise the model including this additional parameter.

Potential developments to the current work include:

- Model each individual hill in the Andes Complex separately. Allocating the hills into separate spatial strata should enable detection of any spatial movement or spatial contractions.

- Develop an observed abundance index with a sub-annual (e.g. seasonal, monthly) time-step. While the vast majority of fisheries analyses are conducted annually, there is no reason why a higher resolution model should not be developed. This would enable a further exploration of hypotheses that reveal finer scale roughy movements, 
for example, disturbance and seasonal spawning migrations.

- Create indicies of abundance for all the ESCR orange roughy subpopulations. This would allow estimation of the recruitment and fish movement between sub-populations, instead of assuming that each sub-population is demographically closed.

- Consider a SPM that accounts for both process and observational errors instead of observational errors alone.

- Model the Andes Complex fishery as two separate populations (population 1: 1991 - 1996 and population 2: 1997 - 2013).

- Apply two of my hypothesised models, for example the Changing Growth Model and the Unweighted Habitat Degradation Model, simultaneously.

- Create a whole ecosystem model of a New Zealand seamount, to examine how the organisms are likely to interact, for example, orange roughy and biogenic habitat, and to assist determining sustainable exploitation regimes.

The effective management of a sustainable fishery requires tracking abundance in some way, such that the total fishing mortality can be adjusted to optimise yield and avoid low stock levels. I have examined the plausibility of a range of hypotheses about the observed hyperdepletion in the orange roughy abundance index of the Andes Seamount Complex. The change in population abundance at the Andes Complex cannot be easily explained by internal population demographics. As a result, I find 
little support for the assumption that the Andes Complex orange roughy stock should be assumed to be demographically closed, although previous stock assessments have indeed done this (Anderson, 2003; McKenzie, 2003; Dunn, 2006) and some areas are still managed in this way, for example, Mercury-Colville and the Cook Canyon (Ministry for Primary Industries, 2014). I have also found evidence suggesting that changes in habitat quality as a consequence of bottom trawling may affect the carrying capacity of the Andes Complex.

Based on my findings here, future assessments of orange roughy stocks (or other species), that are based on CPUE analysis would not be recommended without a thorough understanding of the structure of the wider population. In developing seamount fisheries, it is advised to leave some areas unexploited to preserve potentially valuable habitat (Clark and Dunn, 2012). In established fisheries, I advise quantifying the degree of habitat damage and avoiding exploiting previously unfished areas. A precautionary approach to seamount fisheries is required, due to a lack of understanding of the interactions between the biogenic habitat and fish aggregations. 


\section{Chapter 6}

\section{Appendix}

\subsection{Appendix A: $R$ code}

\subsubsection{Example: Unweighted Habitat Degradation Model}

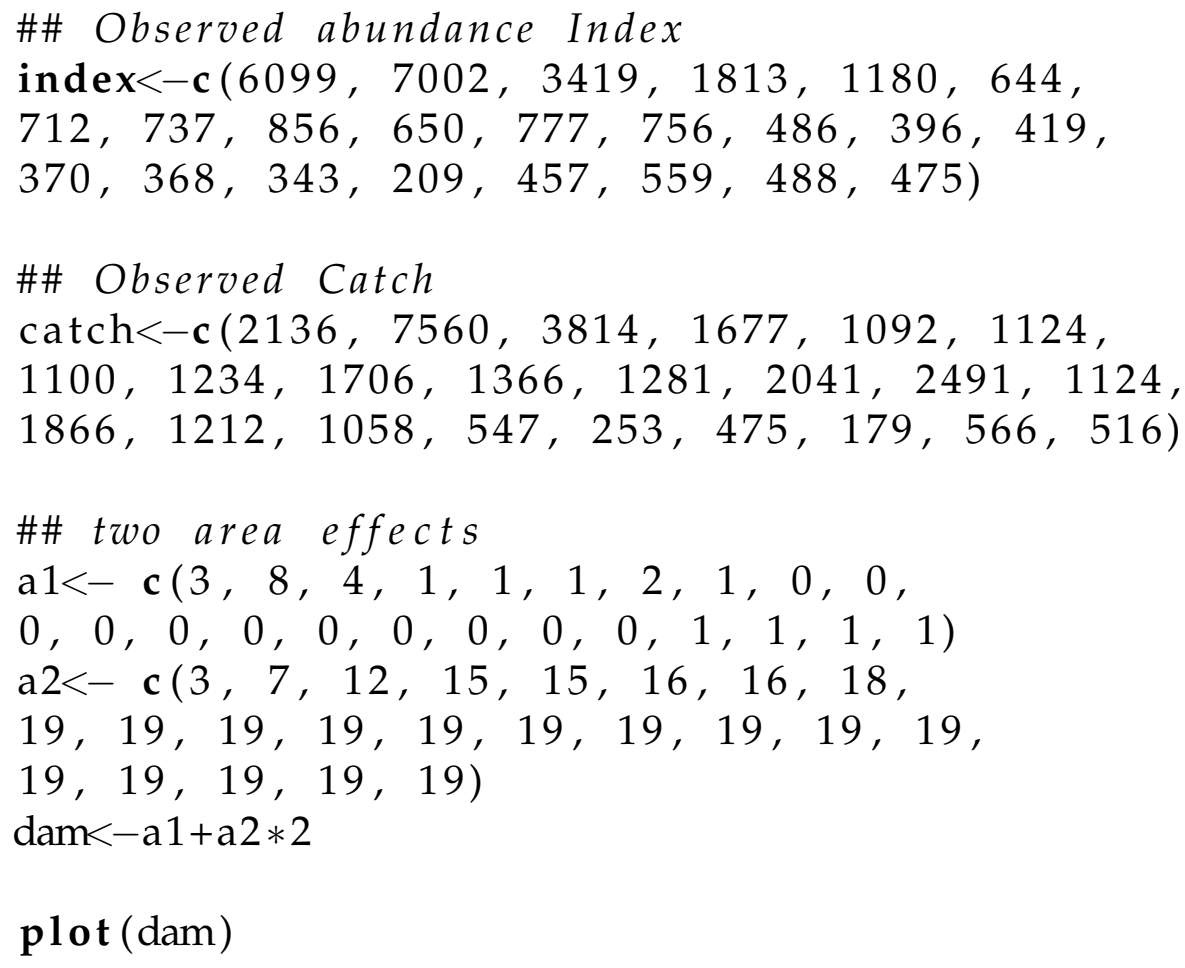




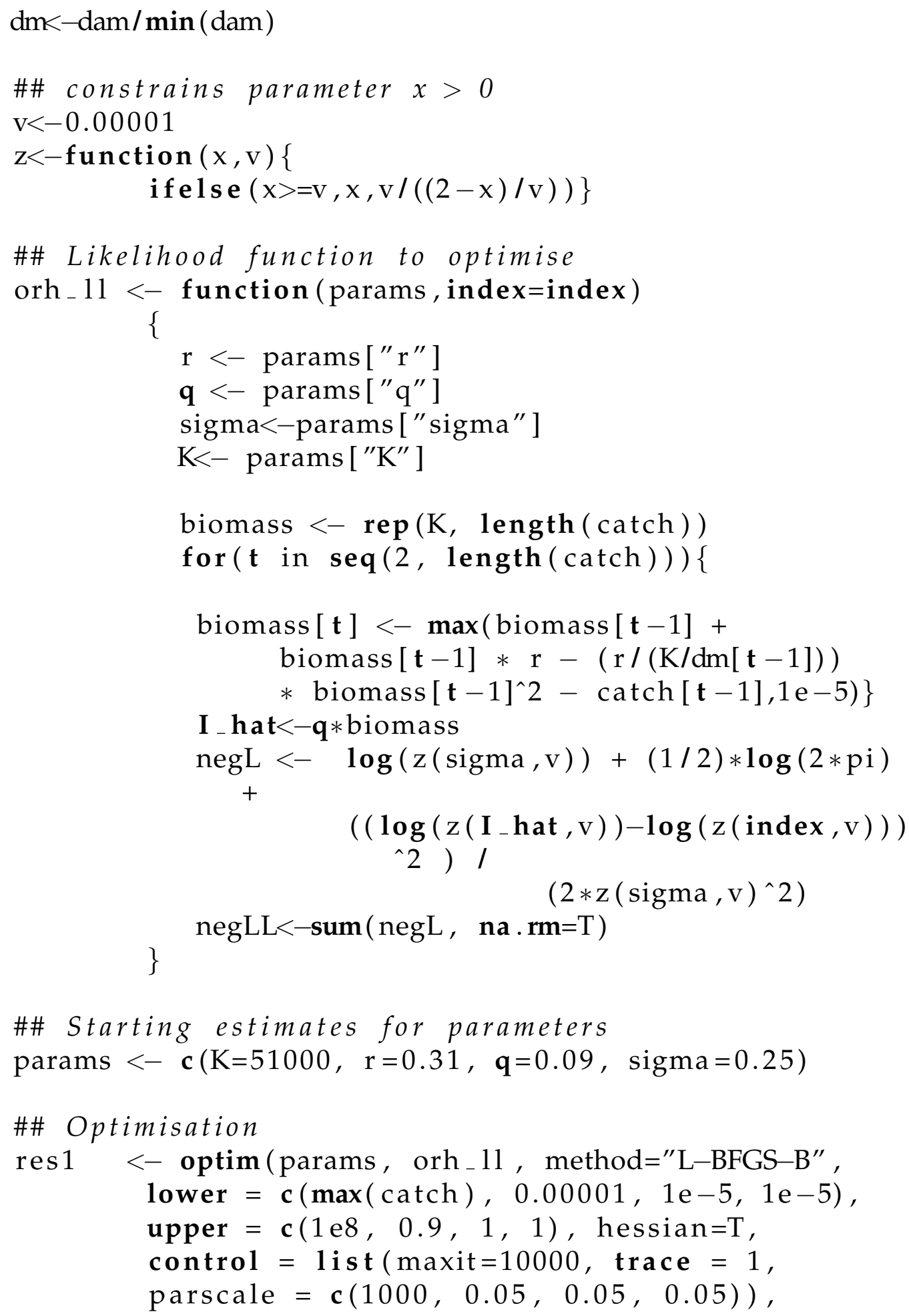




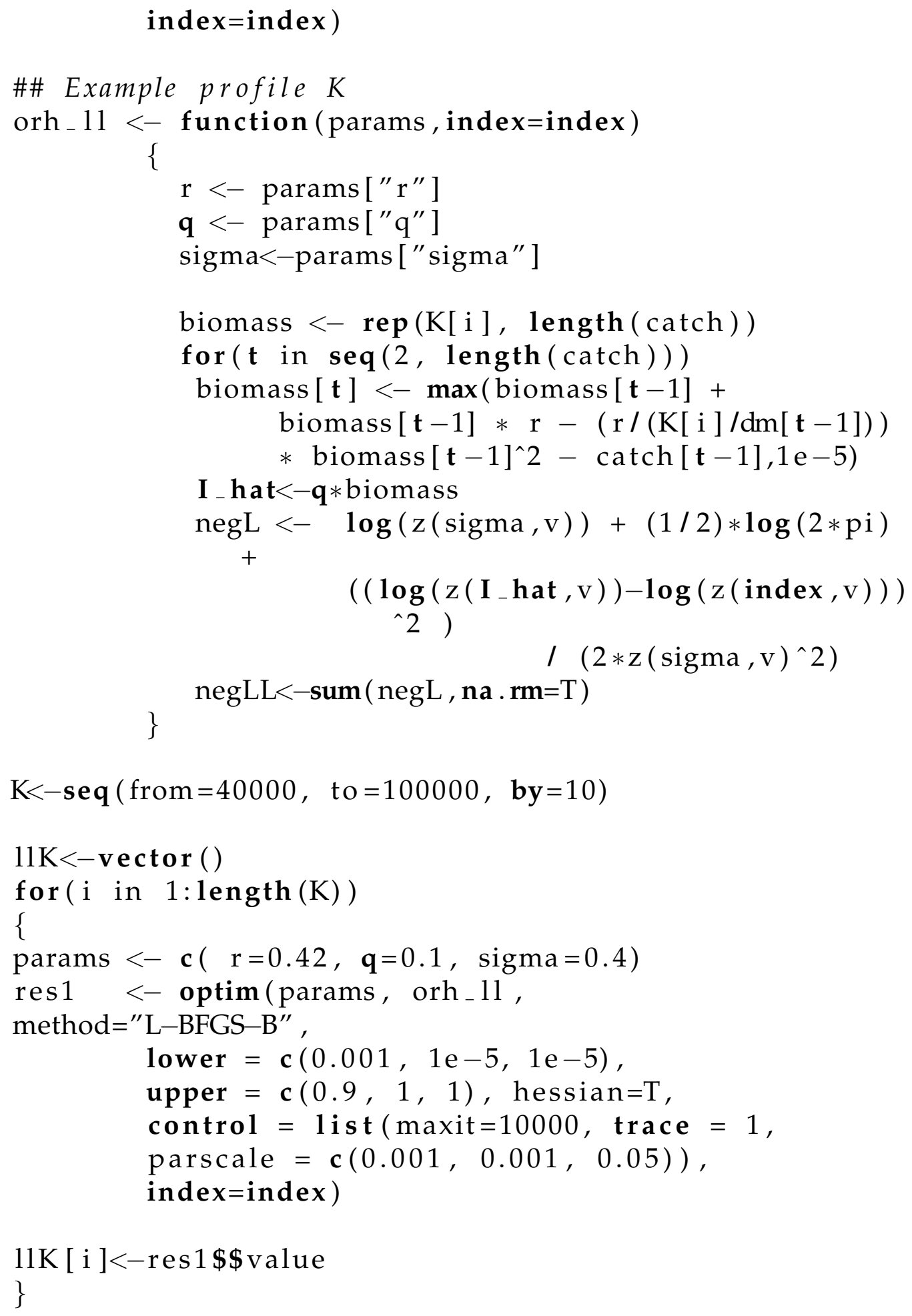




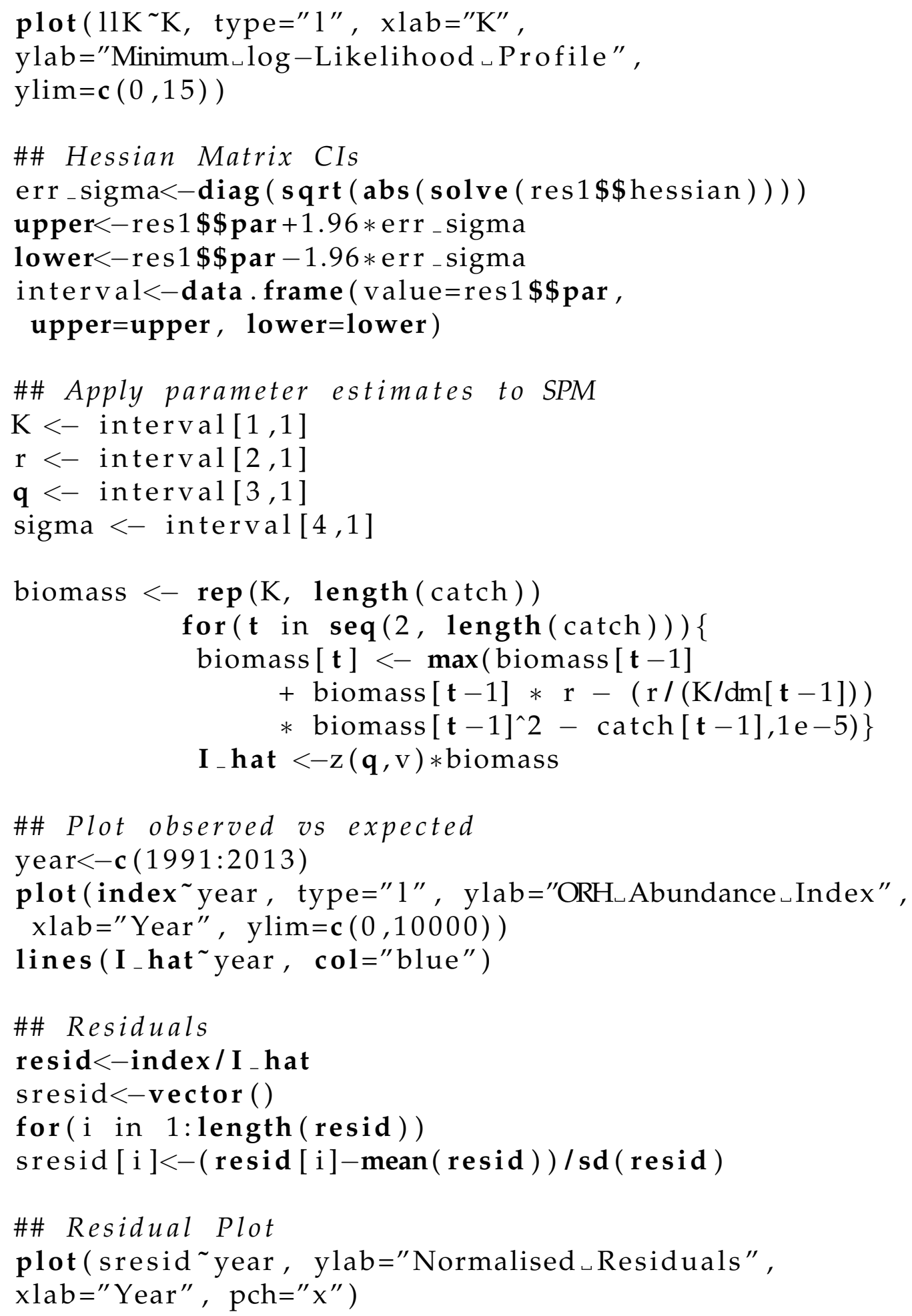




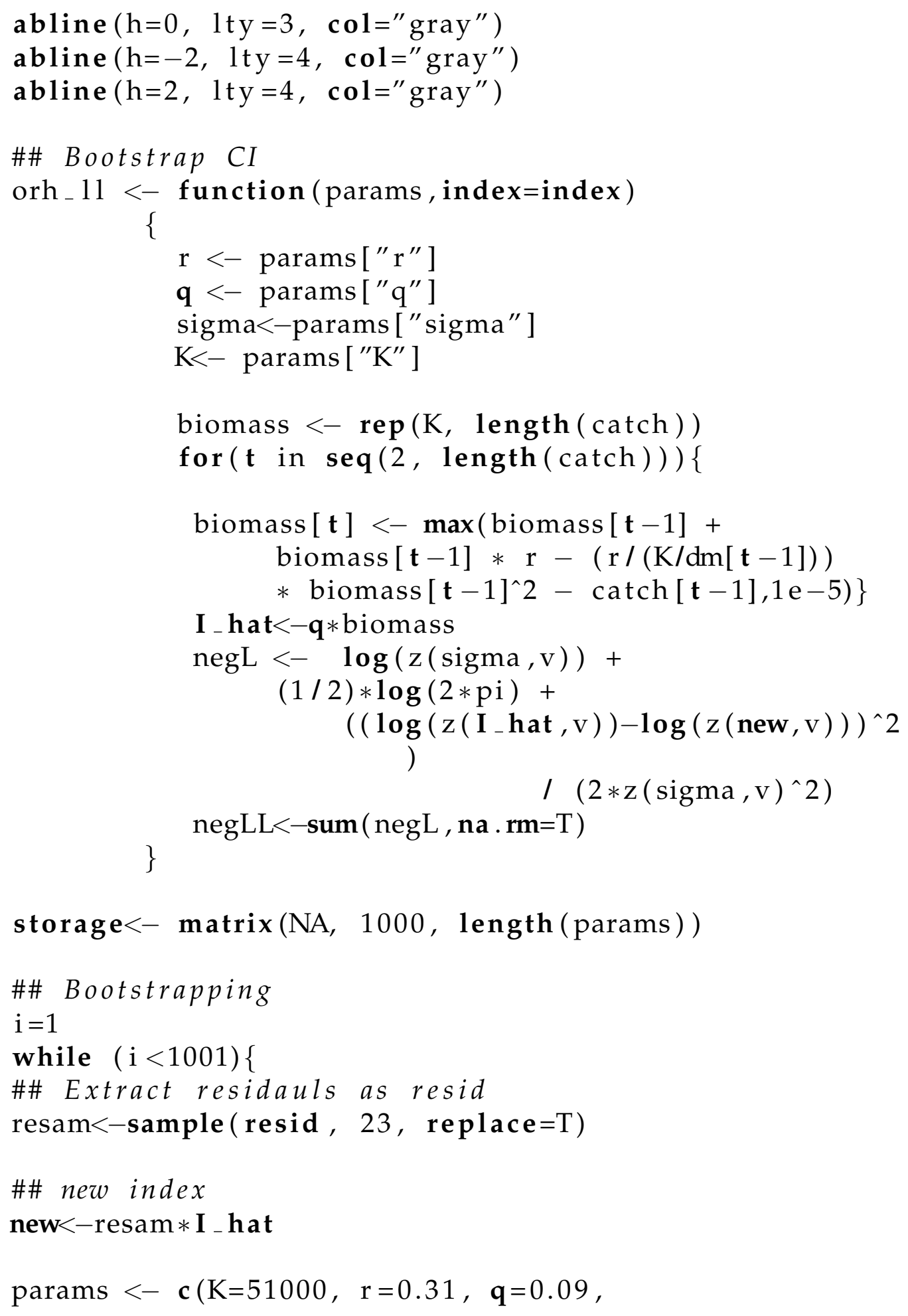




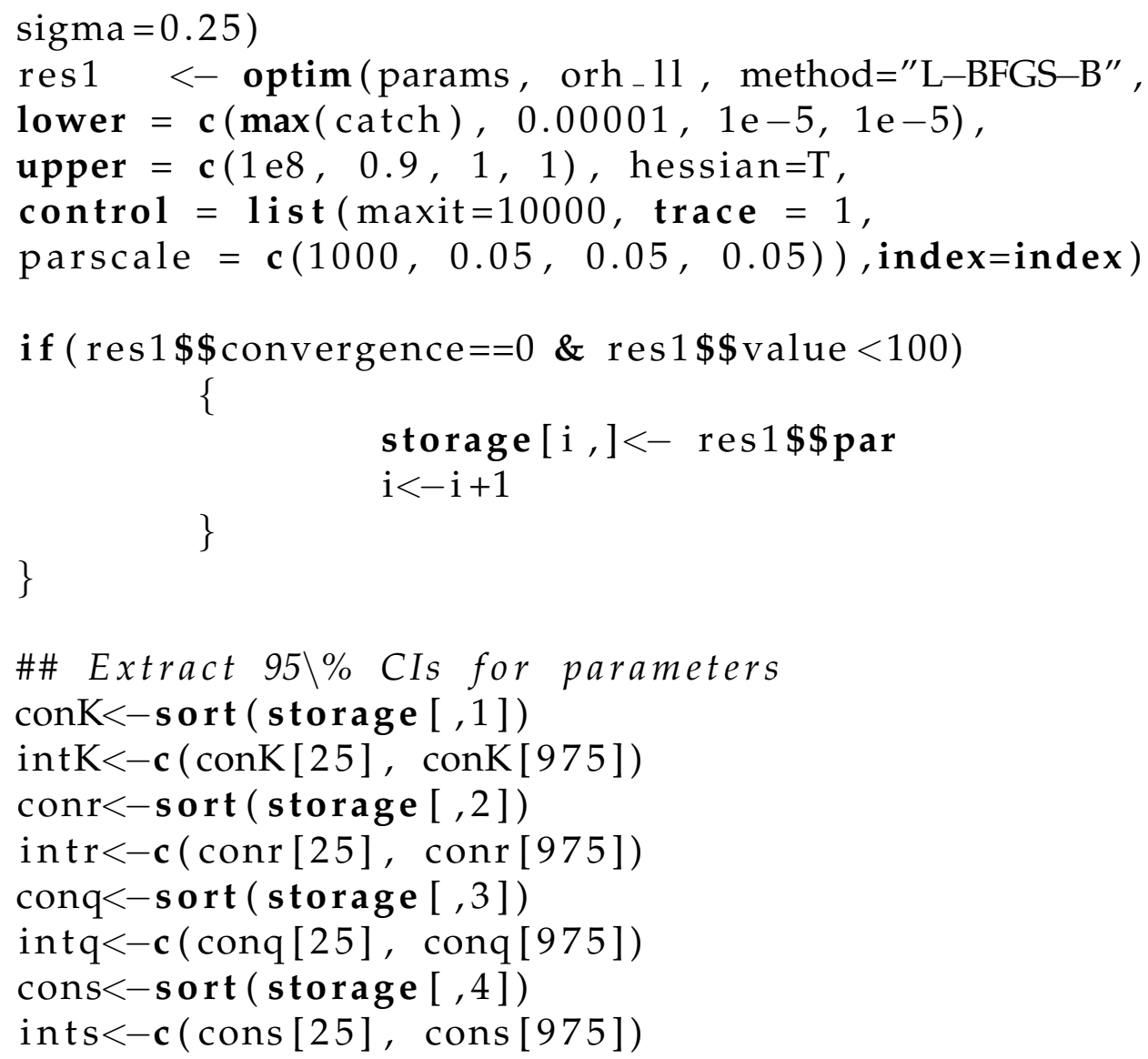




\subsection{Appendix B: Likelihood profiles}

\subsubsection{Changing Growth Model}
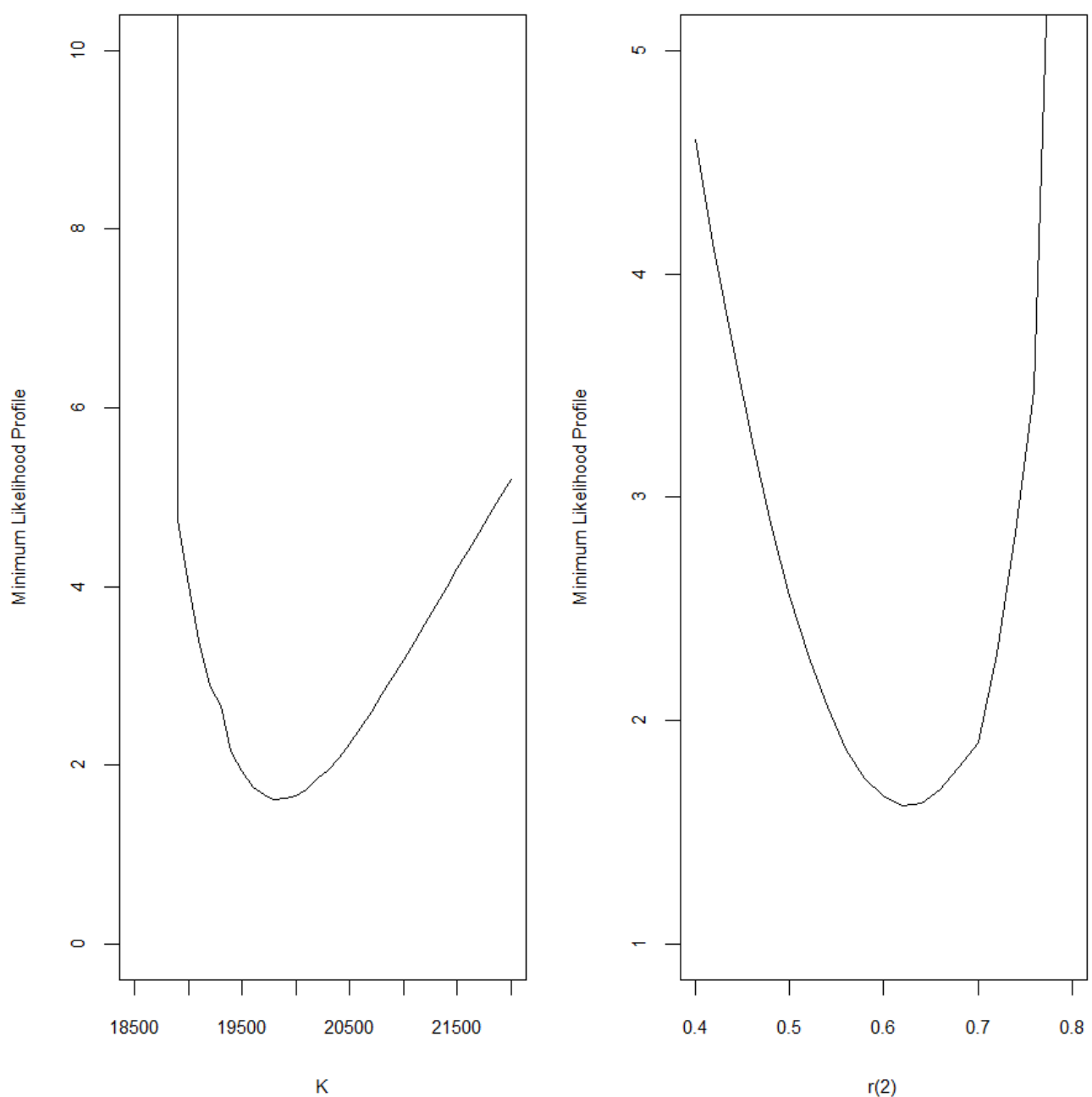

Figure 6.1: Minimum negative log-likelihood profiles for biological parameters $r 2$ and $K$ from the Changing Growth Model ( $r 1$ was $\sim 0$ so it was not profiled). 


\subsubsection{Unweighted Habitat Degradation Model}
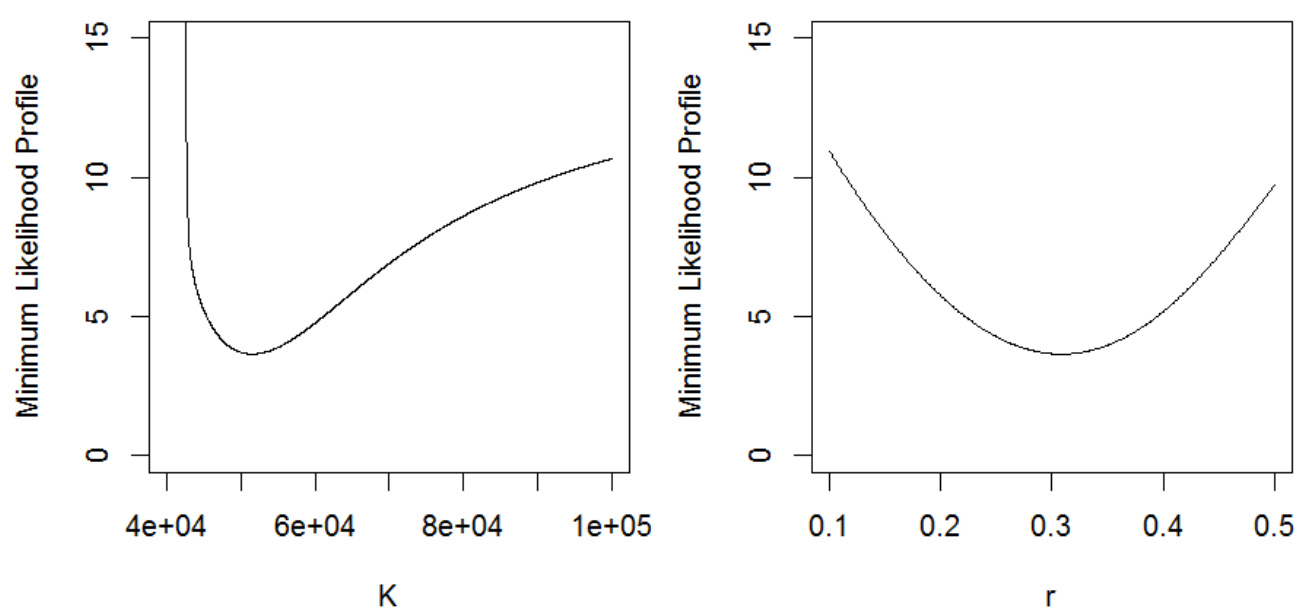

Figure 6.2: Minimum negative log-likelihood profiles for parameters $\mathrm{K}$ and $\mathrm{r}$ for the Unweighted Habitat Degradation Model. 


\section{Bibliography}

Althaus, F., Williams, a., Schlacher, T., Kloser, R., Green, M., Barker, B., Bax, N., Brodie, P., and Hoenlinger-Schlacher, M. (2009). Impacts of bottom trawling on deep-coral ecosystems of seamounts are long-lasting. Marine Ecology Progress Series, 397:279-294.

Anderson, O. and Clark, M. (2003). Analysis of bycatch in the fishery for orange roughy, Hoplostethus atlanticus, on the South Tasman Rise. Marine and Freshwater Research, 54:643-652.

Anderson, O. and Dunn, M. (2012). Descriptive analysis of catch and effort data from New Zealand orange roughy fisheries in ORH 1, 2A, 2B, 3A, 3B, 7A and 7B to the end of the 2008-09 fishing year. New Zealand Fisheries Assessment Report 2012/20, 1584(May).

Anderson, O. F. (2003). CPUE analysis and stock assessment of the East Cape hills (ORH 2a North) orange roughy fishery for 2003. New Zealand Fisheries Assessment Report 2003R4., page 20p.

Bolker, B. M. (2008). Ecological models and data in R. Princeton University Press. 
Bordalo-Machado, P. (2006). Fishing Effort Analysis and Its Potential to Evaluate Stock Size. Reviews in Fisheries Science, 14(4):369-393.

Branch, T. (2001). A review of orange roughy Hoplostethus atlanticus fisheries, estimation methods, biology and stock structure. South African Journal of Marine Science, 23(1):181-203.

Burnham, K. P. and Anderson, D. R. (2002). Model selection and multimodel inference: a practical information-theoretic approach. Springer Science \& Business Media.

Butterworth, D. and Brandao, A. (2005). Some insights into the estimability of non-linear dependence of CPUE on abundance in orange roughy fisheries, based upon a simple age-aggregated production model. New Zealand Ministry of Fisheries review of methods and data used in orange roughy stock assessments, Wellington, October 2005 (unpublished).

Cadrin, S. X., Kerr, L. A., and Mariani, S. (2013). Stock identification methods: applications in fishery science. Academic Press.

Campbell, R. A. (2004). CPUE standardisation and the construction of indices of stock abundance in a spatially varying fishery using general linear models. Fisheries Research, 70(2-3):209-227.

Campbell, R. A. (2015). Constructing stock abundance indices from catch and effort data: Some nuts and bolts. Fisheries Research, 161:109-130.

Canadian Department of Fisheries (2013). Stock assessment of northern 
$(2 J 3 K L)$ cod in 2013. Department of Fisheries and Oceans Science Advisory Sector. DFO, 2014/014.(July):1-27.

Clark, M. (1999). Fisheries for orange roughy (Hoplostethus atlanticus ) on seamounts in New Zealand. Oceanologica Acta, 22:593-602.

Clark, M. (2001). Are deepwater fisheries sustainable?the example of orange roughy (Hoplostethus atlanticus) in New Zealand. Fisheries Research, 51:123-135.

Clark, M. (2010). Effects of Trawling on Seamounts. Oceanography, 23(1):132-133.

Clark, M., Dunn, M., and Anderson, O. (2010). Development of estimates of biomass and sustainable catches for orange roughy fisheries in the New Zealand region outside the EEZ: CPUE analyses, and application of the seamount meta-analysis approach. New Zealand Fisheries Assessment Report, 19:47.

Clark, M. and O'Driscoll, R. (2003). Deepwater fisheries and aspects of their impact on seamount habitat in New Zealand. Journal of Northwest Atlantic Fishery Science, 31:441-458.

Clark, M. R. (1996). Biomass estimation of orange roughy: a summary and evaluation of techniques for measuring stock size of a deep-water fish species in New Zealand. Journal of Fish Biology, 49:114-131.

Clark, M. R. (2009). Deep sea seamount fisheries: a review of global status and future prospects. Latin American Journal of Aquatic Research, 37(3):501-512. 
Clark, M. R., Anderson, O. F., Chris Francis, R. I. C., and Tracey, D. M. (2000). The effects of commercial exploitation on orange roughy (Hoplostethus atlanticus) from the continental slope of the Chatham Rise, New Zealand, from 1979 to 1997. Fisheries Research, 45(3):217-238.

Clark, M. R. and Dunn, M. R. (2012). Spatial management of deep-sea seamount fisheries: balancing sustainable exploitation and habitat conservation. Environmental Conservation, 39(03):204-214.

Clark, M. R. and Rowden, A. A. (2009). Effect of deepwater trawling on the macro-invertebrate assemblages of seamounts on the Chatham Rise, New Zealand. Deep-Sea Research Part I: Oceanographic Research Papers, 56(9):1540-1554.

Clark, M. R. and Tittensor, D. P. (2010). An index to assess the risk to stony corals from bottom trawling on seamounts. Marine Ecology, 31(s1):200211.

Coburn, R. and Doonan, I. J. (1994). Orange roughy on the northeast Chatham Rise: a description of the commercial fishery, 1979-88. MAF Fisheries.

Cope, J. M. and Punt, A. E. (2011). Reconciling stock assessment and management scales under conditions of spatially varying catch histories. Fisheries Research, 107(1-3):22-38.

Doonan, I. J., Coburn, R. P., and Mcmillan, P. J. (2009). Assessment of OEO 3A black oreo for 2006/07. New Zealand Fisheries Assessment Report, 2009/12(March):45. 
Doonan, I. J., Fu, D., and Dunn, M. R. (2015). Harvest control rules for a sustainable orange roughy fishery. Deep Sea Research Part I: Oceanographic Research Papers, 98:53-61.

Dunn, A., Harley, S., Doonan, I., and Bull, B. (2000). Calculation and interpretation of catch-per-unit-effort (CPUE) indices. New Zealand Fisheries Assessment Report, 1:44.

Dunn, M. and Forman, J. (2011). Hypotheses of spatial stock structure in orange roughy Hoplostethus atlanticus inferred from diet, feeding, condition, and reproductive activity. PloS One, 6(11):e26704.

Dunn, M. R. (2006). CPUE analysis and assessment of the Northeast Chatham Rise orange roughy stock (part of ORH 3B) to the end of the 200304 fishing year. New Zealand Fisheries Assessment Report 2006/59, page 76.

Dunn, M. R. (2011). Investigation of some alternative stock assessment model structures for Mid-East Coast orange roughy. New Zealand Fisheries Assessment Report, 2011/63(December).

Dunn, M. R. and Devine, J. A. (2010). An holistic approach to determining stock structure of orange roughy on the Chatham Rise. New Zealand Fisheries Assessment Report 2010/17, page 35.

Dunn, M. R., Rickard, G. J., Sutton, P. J. H., and Doonan, I. J. (2009). Nursery grounds of the orange roughy around New Zealand. ICES Journal of Marine Science, 66(5):871-885. 
Erisman, B. E., Allen, L. G., Claisse, J. T., Pondella, D. J., Miller, E. F., Murray, J. H., and Walters, C. (2011). The illusion of plenty: hyperstability masks collapses in two recreational fisheries that target fish spawning aggregations. Canadian Journal of Fisheries and Aquatic Sciences, 68(10):1705-1716.

Etnoyer, B. P. J. (2009). Deep-Sea Corals on Seamounts. Oceanography, 23(1):128-129.

Field, K. and Clark, M. (2001). Catch-per-unit-effort (CPUE) analysis and stock assessment for black cardinalfish (Epigonus telescopus) in QMA 2. New Zealand Fisheries Assessment Report, 23:22.

Francis, C. and Clark, M. (2005). Sustainability issues for orange roughy fisheries. Bulletin of Marine Science, 76(2):337-351.

Francis, R. and Clark, M. (1998). Inferring spawning migrations of orange roughy (Hoplostethus atlanticus) from spawning ogives. Marine and Freshwater Research, 49:103-108.

Gavaris, S. (1980). Use of a multiplicative model to estimate catch rate and effort from commercial data. Canadian Journal of Fisheries and Aquatic Sciences, 37(12):2272-2275.

Haddon, M. (2010). Modelling and quantitative methods in fisheries. CRC press.

Harley, S. J., Myers, R. A., and Dunn, A. (2001). Is catch-per-unit-effort proportional to abundance? Canadian Journal of Fisheries and Aquatic Sciences, 58(9):1760-1772. 
Hawkins, D. M. (2004). The Problem of Overfitting. Journal of Chemical Information and Computer Sciences, 44(1):1-12.

Hicks, A. C. (2013). The utility of catch-per-unit-effort when assessing and managing long-lived fish stocks. PhD thesis, University of Washington.

Hilborn, R. (1985). Fleet dynamics and individual variation: why some people catch more fish than others. Canadian Journal of Fisheries and Aquatic Sciences, 42:2-13.

Hilborn, R. and Mangel, M. (1997). The ecological detective: Confronting models with data. Monographs in Population Biology, 28:336.

Hilborn, R. and Walters, C. (1987). A general model for simulation of stock and fleet dynamics in spatially heterogeneous fisheries. Canadian Journal of Fisheries and Aquatic Sciences, 44(7):1366-1369.

Hilborn, R. and Walters, C. (1992). Quantitative fisheries stock assessment: Choice, dynamics $\mathcal{E}$ uncertainty. . Chapman and Hill, New York.

Hubbs, C. (1958). Initial discoveries of fish faunas on seamounts and offshore banks in the eastern Pacific. Pacific Science, 13:311-316.

Hutchings, J. A. (1996). Spatial and temporal variation in the density of northern cod and a review of hypotheses for the stock's collapse. Canadian Journal of Fisheries and Aquatic Sciences, 53(5):943-962.

Jacobsen, L., Baktoft, H., Jepsen, N., Aarestrup, K., Berg, S., and Skov, C. (2014). Effect of boat noise and angling on lake fish behaviour. Journal of Fish Biology, 84(6):1768-80. 
Jones, J. B. (1992). Environmental impact of trawling on the seabed: A review. New Zealand Journal of Marine and Freshwater Research, 26(1):5967.

Knowles, W., Dunn, A., Bull, B., Smith, M., and Manning, M. (2012). nzPlot: Plot NZ coastline and ocean features. R package version 0.33.

Koslow, J. (2000). Continental slope and deep-sea fisheries: implications for a fragile ecosystem. ICES Journal of Marine Science, 57(3):548-557.

Koslow, J., Gowlett-Holmes, K., Lowry, J. K., O’Hara, T., Poore, G. C. B., and Williams, a. (2001). Seamount benthic macrofauna off southern Tasmania: Community structure and impacts of trawling. Marine Ecology Progress Series, 213:111-125.

Koslow, J., Kloser, R., and Stanley, C. (1995). Avoidance of a camera system by a deepwater fish, the orange roughy (Hoplostethus atlanticus). Deep Sea Research Part I: Oceanographic Research Papers, 42(2):233-244.

Large, K. (2013). Population changes in rattail species on the Chatham Rise. Master's thesis, Victoria University of Wellington.

MacKay, A. K., Wood, B. A., and Clark, M. R. (2005). Chatham Rise Bathymetry. NIWA Miscellaneous Chart Series No.82.

Marchal, P., Andersen, B., Caillart, B., Eigaard, O., Guyader, O., Hovgaard, H., Iriondo, A., Fur, F. L., Sacchi, J., Santurtu, M., Ifremer, P. M., Sea, N., Gambetta, Q., Andersen, F. B., Eigaard, O., Difres, H. H., Castle, C., Charlottenlund, D. K., Caillart, D. B., Fur, F. L., Developpement, O., 
Iriondo, F. A., Moros, Z. I., Ifremer, F. O. G., and Ugarte, T. (2007). Impact of technological creep on fishing effort and fishing mortality, for a selection of European fleets. ICES Journal of Marine Science, 64(1):192209.

Martín, J., Puig, P., Palanques, A., and Ribó, M. (2014). Trawling-induced daily sediment resuspension in the flank of a Mediterranean submarine canyon. Deep Sea Research Part II: Topical Studies in Oceanography, 104:174-183.

Maunder, M., Sibert, J., Fonteneau, A., Hampton, J., Kleiber, P., and Harley, S. (2006). Interpreting catch per unit effort data to assess the status of individual stocks and communities. ICES Journal of Marine Science, 63(8):1373-1385.

Maunder, M. N. and Punt, A. E. (2004). Standardizing catch and effort data: a review of recent approaches. Fisheries Research, 70(2-3):141-159.

McAllister, M. and Kirchner, C. (2002). Accounting for structural uncertainty to facilitate precautionary fishery management: illustration with Namibian orange roughy. Bulletin of Marine Science, 70(2):499-540.

McKenzie, A. (2003). Standardised CPUE Analysis and Stock Assessment of the Northwest Chatham Rise Orange Roughy Stock (part of ORH 3B). Ministry of Fisheries.

McKenzie, A. and Coburn, R. (2009). Stock assessment of smooth oreo in the bounty plateau study area (part of OEO 6) for 2007-08. New Zealand Fisheries Assessment Report, 42:42. 
Ministry for Primary Industries (2014). Fisheries Assessment Plenary, May 2014: stock assessments and stock status. Technical Report May, Compiled by the Fisheries Science Group, Ministry for Primary Industries, Wellington, New Zealand.

Moore, J. A. (1999). Deep-sea Finfish Fisheries: Lessons from History. Fisheries, 24(7):16-21.

Myers, R. A., Mertz, G., and Fowlow, P. S. (1997). Maximum population growth rates and recovery times for Atlantic cod, Gadus morhua. Fishery Bulletin, 95(4):762-772.

Nash, J. C. and Dalzell, C. (2013). Optimisation in R: A conversation with John Nash about optim() and optimx(). http:/ / www.ibm.com/developerworks/library/ba-optimR-johnnash/index.html.

Nash, J. C. and Varadhan, R. (2011). Unifying optimization algorithms to aid software system users: optimx for R. Journal of Statistical Software, 43(9):1-14.

Niklitschek, E. J., Cornejo-Donoso, J., Oyarzún, C., Hernández, E., and Toledo, P. (2010). Developing seamount fishery produces localized reductions in abundance and changes in species composition of bycatch. Marine Ecology, 31:168-182.

Nishida, T. and Chen, D.-G. (2004). Incorporating spatial autocorrelation into the general linear model with an application to the yellowfin tuna (Thunnus albacares) longline CPUE data. Fisheries Research, 70(2-3):265274. 
Norse, E. a., Brooke, S., Cheung, W. W. L., Clark, M. R., Ekeland, I., Froese, R., Gjerde, K. M., Haedrich, R. L., Heppell, S. S., Morato, T., Morgan, L. E., Pauly, D., Sumaila, R., and Watson, R. (2012). Sustainability of deep-sea fisheries. Marine Policy, 36(2):307-320.

O'Driscoll, R. and Joux, P. D. (2012). Species identification in seamount fish aggregations using moored underwater video. ICES Journal of Marine Science.

O'Driscoll, R. L. and Clark, M. R. (2005). Quantifying the relative intensity of fishing on New Zealand seamounts. New Zealand Journal of Marine and Freshwater Research, 39(4):839-850.

Pankhurst, N. W. (1988). Spawning dynamics of orange roughy, Hoplostethus atlanticus, in mid-slope waters of New Zealand. Environmental Biology of Fishes, 21(2):101-116.

Pitcher, T. J., Clark, M. R., Morato, T., and Watson, R. (2010). Seamount fisheries: Do they have a future? Oceanography, 23(1):134.

Priede, I. G., Bergstad, O. A., Miller, P. I., Vecchione, M., Gebruk, A., Falkenhaug, T., Billett, D. S., Craig, J., Dale, A. C., Shields, M. A., et al. (2013). Does presence of a mid-ocean ridge enhance biomass and biodiversity? PLoS One, e61550.

Probert, P. K., McKnight, D., and Grove, S. L. (1997). Benthic invertebrate bycatch from a deepwater trawl fishery, Chatham Rise, New Zealand. Aquatic Conservation: Marine and Freshwater Ecosystems, 7(1):27-40. 
Punt, A. E. (2003). Extending production models to include process error in the population dynamics. Canadian Journal of Fisheries and Aquatic Sciences, 60(10):1217-1228.

R Core Team (2015). R: A Language and Environment for Statistical Computing. R Foundation for Statistical Computing, Vienna, Austria. ISBN 3-900051-07-0.

Rindorf, A. and Andersen, B. S. 1. (2008). Do North Sea cod (Gadus morhua) fisheries maintain high catch rates at low stock size? Canadian Journal of Fisheries and Aquatic Sciences, 65(9):1800-1813.

Roa-Ureta, R. H. (2012). Modelling in-season pulses of recruitment and hyperstability-hyperdepletion in the Loligo gahi fishery around the Falkland Islands with generalized depletion models. ICES Journal of Marine Science, 69(8):1403-1415.

Roberts, C. (2002). Deep impact: the rising toll of fishing in the deep sea. Trends in Ecology \& Evolution, 17(5):242-245.

Rose, G. and Kulka, D. (1999). Hyperaggregation of fish and fisheries: how catch-per-unit-effort increased as the northern cod (Gadus morhua) declined. Canadian Journal of Fisheries and Aquatic Sciences, 56(S1):118127.

Rowden, A. A., Dower, J. F., Schlacher, T. A., Consalvey, M., and Clark, M. R. (2010). Paradigms in seamount ecology: fact, fiction and future. Marine Ecology, 31:226-241. 
Schaefer, M. B. (1954). Some aspects of the dynamics of populations important to the management of the commercial marine fisheries. InterAmerican Tropical Tuna Commission Bulletin, 1(2):23-56.

Seber, G. (1982). The estimation of animal abundance and related parameters. MacMillan Publishing, New York.

Sissenwine, M. and Mace, P. (2007). Can deep water fisheries be managed sustainably? FAO Fisheries Report, 838:61-111.

Staudigel, H., Koppers, A. A., Lavelle, J. W., Pitcher, T. J., and Shank, T. M. (2010). Defining the word seamount. Oceanography, 23.

Tracey, D. M., Bull, B., Clark, M. R., and MaCkay, K. a. (2004). Fish species composition on seamounts and adjacent slope in New Zealand waters. New Zealand Journal of Marine and Freshwater Research, 38(1):163-182.

Walters, C. (2003). Folly and fantasy in the analysis of spatial catch rate data. Canadian Journal of Fisheries and Aquatic Science, 60:1433-1436.

Walters, C. and Maguire, J. (1996). Lessons for stock assessment from the northern cod collapse. Reviews in Fish Biology and Fisheries, 6:125-137.

Wiff, R., Quiroz, J. C., Gatica, C., Contreras, F., Paramo, J., and Barrientos, M. A. (2012). Uncertain population dynamic and state variables of alfonsino (Beryx splendens). Latin American Journal of Aquatic Research, 40(1):201-212.

Wilberg, M. J., Thorson, J. T., Linton, B. C., and Berkson, J. (2009). Incorporating Time-Varying Catchability into Population Dynamic Stock Assessment Models. Reviews in Fisheries Science, 18(1):7-24. 